\title{
Phosphorylation of Synaptotagmin 4 \\ captures transiting dense core vesicles at active synapses
}

\author{
Dissertation \\ for the award of the degree \\ "Doctor rerum naturalium" (Dr.rer.nat.) \\ of the Georg-August-Universität Göttingen \\ within the IMPRS Neuroscience program \\ of the Georg-August University School of Science (GAUSS)
}

submitted by

Vinita Bharat

from Delhi, India

Göttingen, 2016 


\title{
Thesis Committee
}

\author{
Camin Dean,PhD \\ Department of Trans-Synaptic Signaling \\ (Reviewer) \\ European Neuroscience Institute, Göttingen \\ Prof. Dr.rer.nat.Nils Brose \\ Department of Molecular Neurobiology \\ (Reviewer) \\ Max Planck Institute of Experimental Medicine \\ Göttingen \\ Prof. Dr. Reinhard Jahn \\ Department of Neurobiology \\ Max Planck Institute for Biophysical Chemistry \\ Göttingen
}

\section{Members of the Examination Board}
Camin Dean,PhD
Department of Trans-Synaptic Signaling
(Reviewer)
European Neuroscience Institute, Göttingen
Prof. Dr.rer.nat.Nils Brose
Department of Molecular Neurobiology
(Reviewer)
Max Planck Institute of Experimental Medicine
Göttingen

\section{Further members of the Examination Board}

Prof. Dr. Reinhard Jahn

Prof. Dr.Thomas Dresbach

Prof. Dr. Michael Hörner

Prof. Dr. Ralf Heinrich
Department of Neurobiology

Max Planck Institute for Biophysical Chemistry Göttingen

Department of Anatomy and Embryology University Medical Centre Göttingen

Department of Cellular Neurobiology European Neuroscience Institute Göttingen

Department of Neurobiology Schwann-Schleiden Research Centre Göttingen 


\section{Affidavit}

I hereby declare that the presented thesis entitled "Phosphorylation of Synaptotagmin 4 captures transiting dense core vesicle at active synapses" has been written independently and with no other sources and aids than quoted.

Göttingen, $21^{\text {st }}$ March 2016

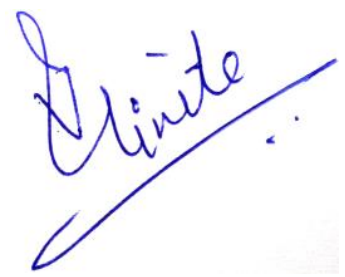

Vinita Bharat 



\section{Abstract}

Synaptic modulation requires fast recruitment of neuronal dense core vesicles (DCVs) containing various neuropeptides and neurotrophins at nerve terminals. DCVs undergo long-range trafficking in axons to deliver cargoes at release sites. However, the question of whether and how specific sites capture these transiting vesicles upon neuronal activity is open. In this study, we have used a Synaptotagmin (Syt) isoform, Syt4, as a DCV marker to investigate trafficking and activity-dependent capture of DCVs in hippocampal neurons. We found that Syt4-harboring vesicles are highly mobile on microtubules and switch directions only at the distal end of axons in hippocampal neurons. We examined the effects of phosphorylation of Syt4 at S135 on trafficking, capture and fusion of DCVs in mature neurons. We found that phosphomimetic Syt4 vesicles traffic less and are more concentrated at synapses. Conversely, phosphodeficient Syt4 vesicles had the most processivity and were least localized at synapses. We also found that disrupting actin, which is enriched at pre-synaptic sites, enhances the mobility of phosphomimetic vesicles. We found that the motor protein Kif1A is associated with Syt4 vesicles but phosphomimetic vesicles had less interaction with Kif1A. Over-expression of Kif1A rescued the trafficking of phosphomimetic Syt4 vesicles. In addition, we found that c-Jun N-terminal kinase (JNK) phosphorylates Syt4 at S135 specifically causing decreased motility of transiting DCVs. Furthermore, increased neuronal activity promoted capture of transiting vesicles at synapses via a JNK phosphorylation dependent mechanism. Phosphorylation of Syt4 did not affect the fusion of vesicles at synaptic and non-synaptic sites in hippocampal neurons. Together, this study reveals a JNK-dependent phosphorylation mechanism involved in trafficking and capture of Syt4 harboring DCVs in hippocampal neurons. We propose a mechanism whereby JNK at active synapses phosphorylates Syt4 at S135 on transiting DCVs, promoting destabilization of Syt4-Kif1A binding and allowing capture of DCVs at synapses by actin. This mechanism would potentially allow fast recruitment of dense core vesicles to active synapses, ensuring the efficient delivery of neuropeptides and neurotrophins to specific sites in hippocampal neurons whenever needed. 



\section{Table of Contents}

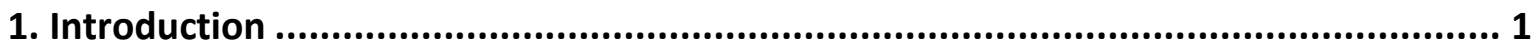

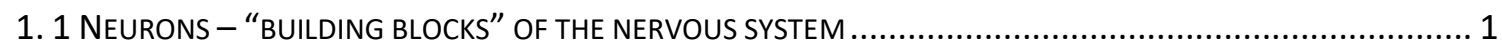

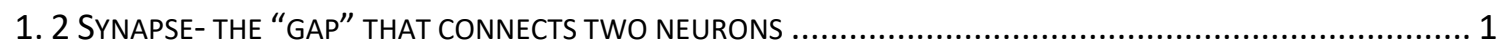

1. 3 NEUROSECRETORY VESICLES - "VEHICLES" FOR NEUROTRANSMISSION .............................................. 2

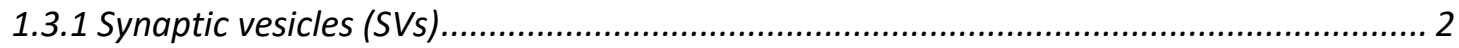

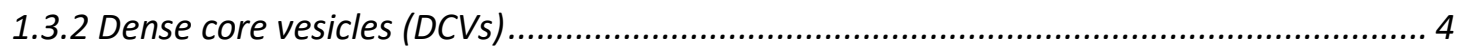

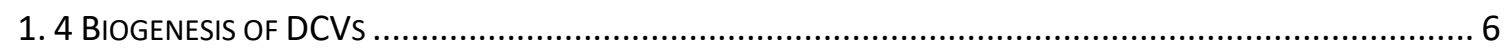

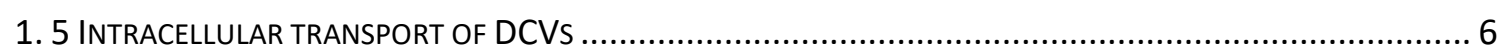

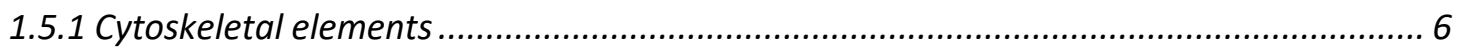

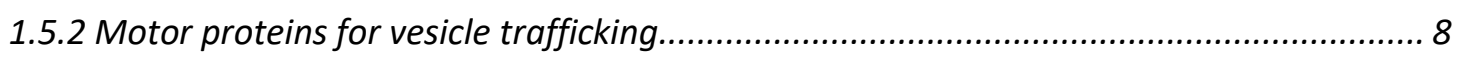

1. 6 KINESIN-3 FAMILY, UNC-104/KIF1A: A MOTOR PROTEIN FOR DCVS................................................. 9

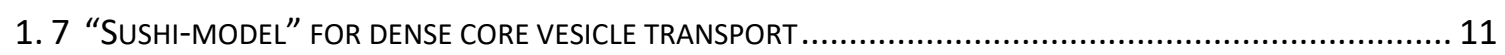

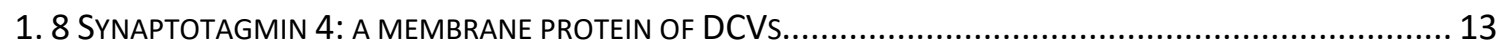

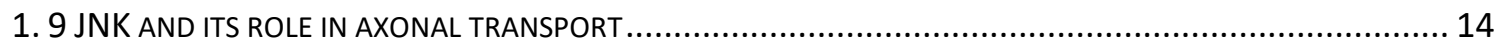

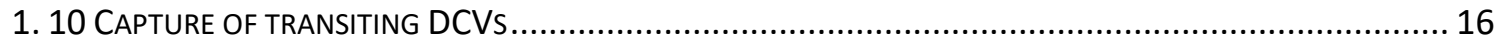

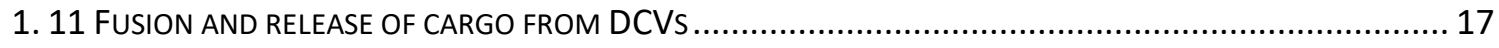

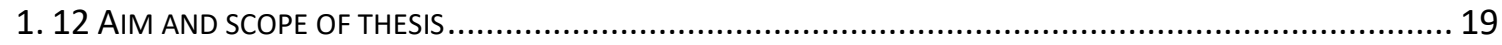

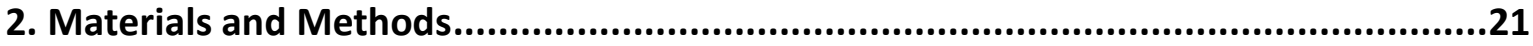

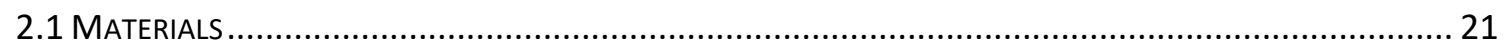

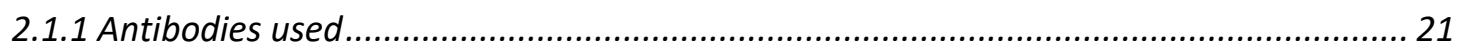

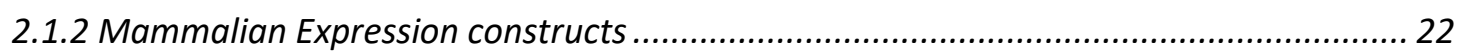

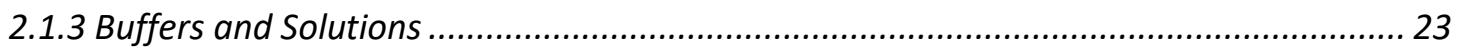

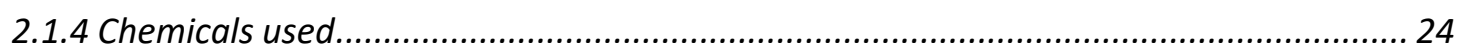

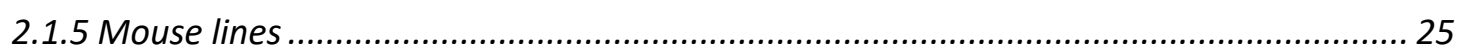

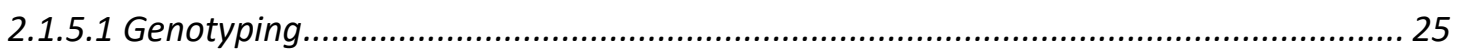

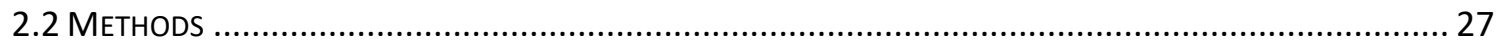

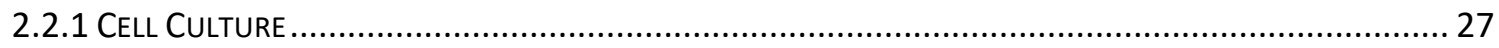

2.2.1.1 Dissociated rat hippocampal neuron culture preparation .......................................... 27

2.2.1.2 Dissociated mouse hippocampal neuron culture preparation ...................................... 28

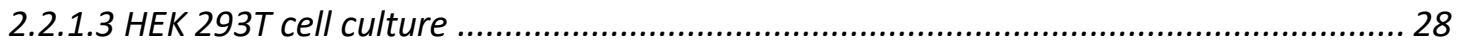

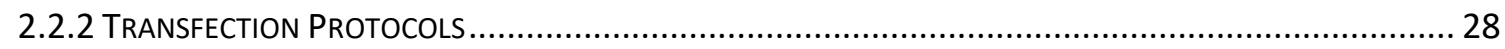

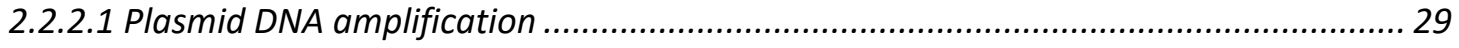




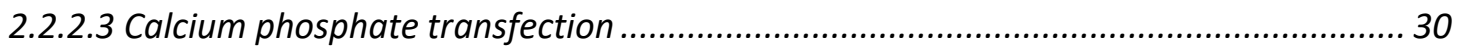

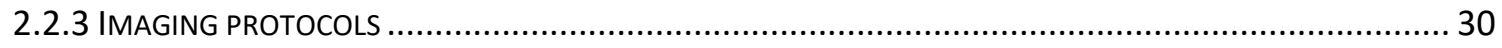

2.2.3.1 Immunocytochemistry (ICC) and fixed sample imaging ............................................ 30

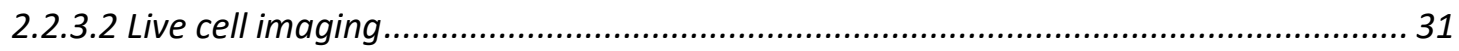

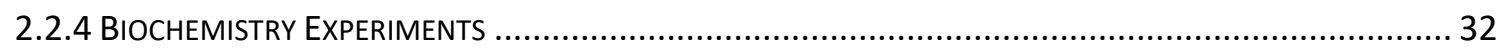

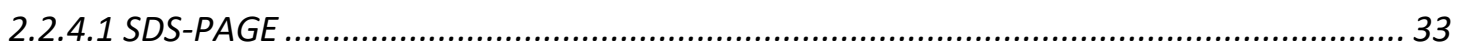

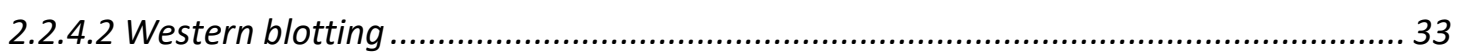

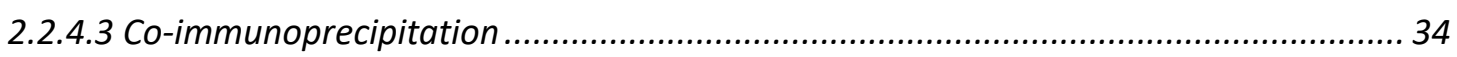

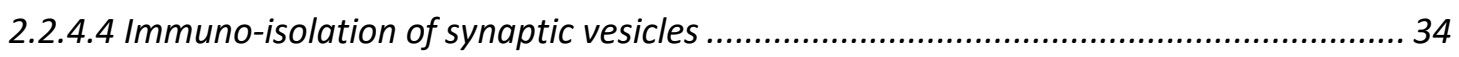

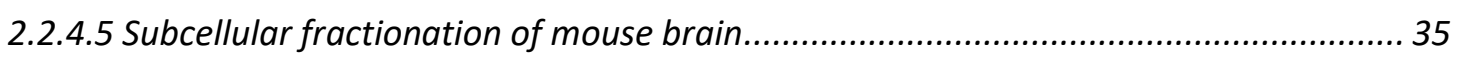

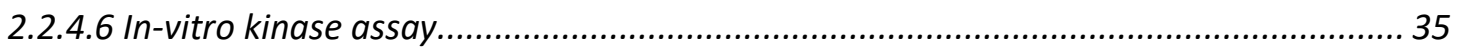

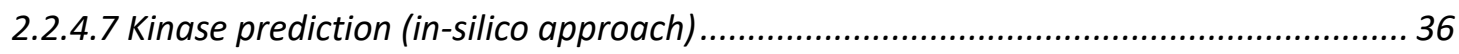

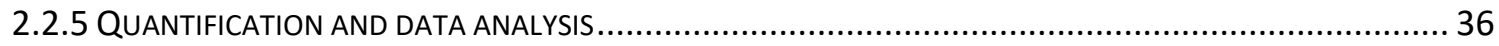

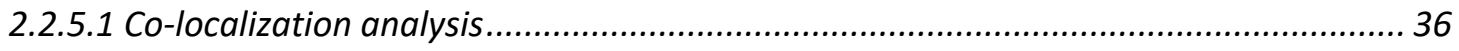

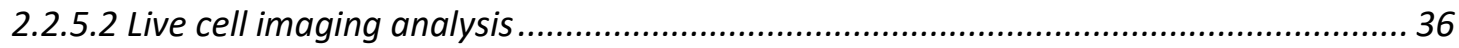

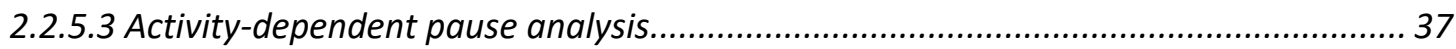

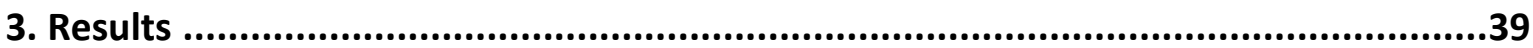

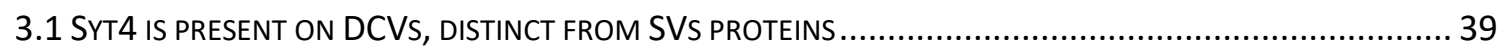

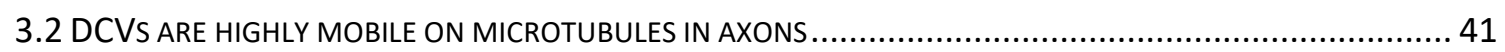

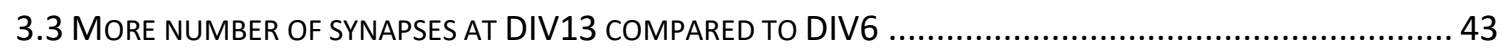

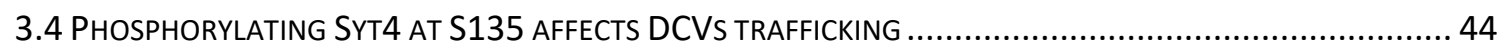

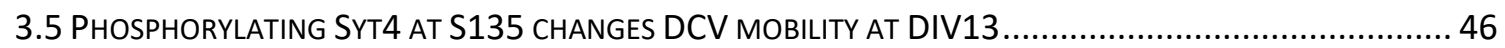

3.6 S135E VESICLES ARE LEAST MOBILE AND S135A VESICLES ARE HIGHLY MOBILE IN AXONS .........................49

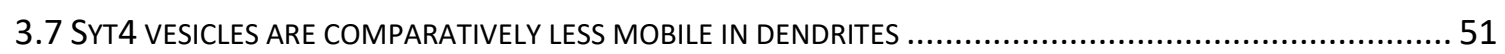

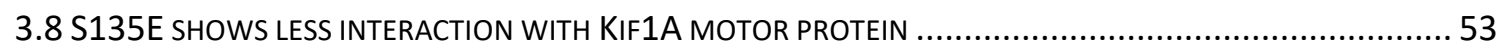

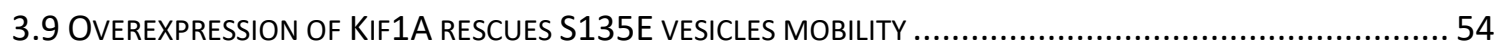

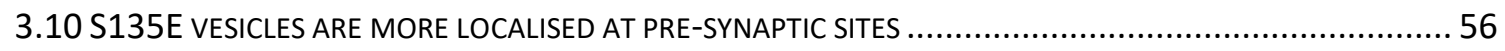

3.11 DISRUPTING ACTIN INCREASES THE MOBILITY OF PHOSPHORYLATED VESICLES AND DOES NOT CHANGE

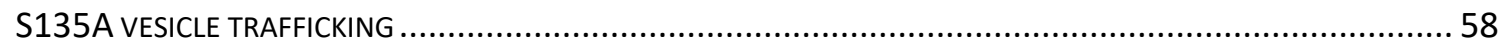

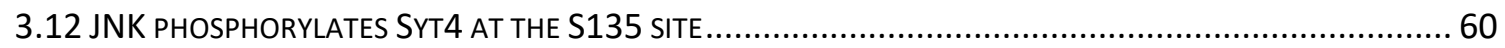

3.13 Phosphorylation Of JNK AT SYT4 S135 DECREASES THE TRAFFICKING OF DCVs ............................. 61

3.14 PHOSPHORYLATION OF SYT4 S135 BY OVER-EXPRESSING JNK ALSO REDUCES THE TRAFFICKING OF DCVS64

3.15 ACTIVITY-DEPENDENT PHOSPHORYLATION OF S135 CAPTURES TRANSITING SYT4 VESICLES..................... 66

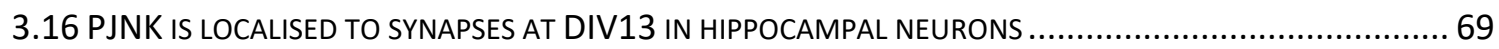


3.18 PhOSPHORYLATION OF SYT4 AT S135 DOESN'T CHANGE THE FUSION OF DCVS AT SYNAPTIC OR NON-

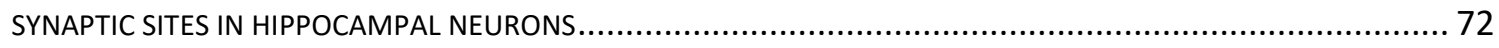

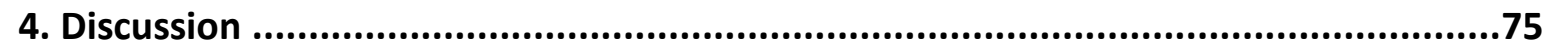

4.1 LONG RANGE TRAFFICKING OF SYT4 HARBORING DENSE CORE VESICLES ON MICROTUBULES OF HIPPOCAMPAL

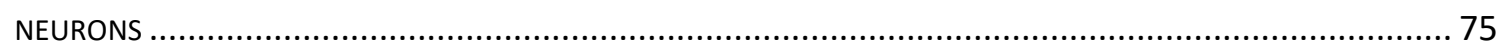

4.1.1. "Sushi-Model" of DCV trafficking in hippocampal neurons ....................................... 76

4.2 JNK, THE KINASE RESPONSIBLE FOR PHOSPHORYLATING SYT4 AT S135 ....................................... 77

4.3 PHOSPHORYLATION OF SYT4 AT S135 DECREASES DCV TRAFFICKING IN MATURE NEURONS ...................78

4.3.1 Phosphorylation of Syt4 at S135 reduces DCV mobility developmentally .................... 78

4.3.2 Phosphorylated Syt4 vesicles show reduced interaction with the motor protein Kif1A. 79

4.3.3 Over-expression of Kif1A rescues the trafficking of phosphorylated vesicles............... 80

4.4 ACTIVITY DEPENDENT JNK PHOSPHORYLATION CAPTURES SYT4 VESICLES AT SYNAPSES ........................80

4.4.1 Actin helps in capturing phosphorylated Syt4 vesicles at pre-synaptic sites ................ 80

4.4.2 JNK dependent capture of Syt4 vesicles at active synapses................................... 81

4.6 EFFECT OF PHOSPHORYLATING SYT4 AT S135 ON THE FUSION PROPERTIES OF DCVS ........................8 83

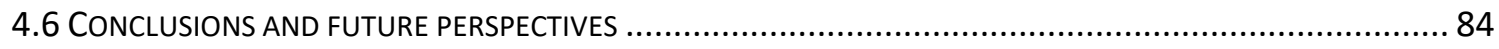

5. References............................................................................................87

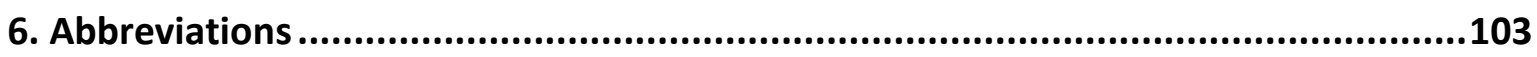

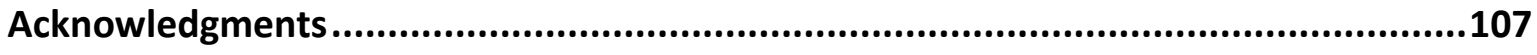

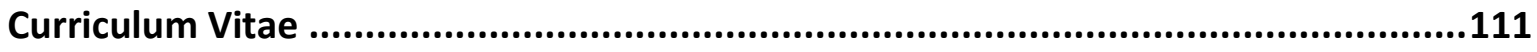




\section{List of figures}

Figure 1.1 Co-existence of SVs and DCVs in the chemical synapse........................................ 3

Figure 1.2 Actin and microtubule architecture in a neuron .................................................. 7

Figure 1.3 Molecular mechanism of Kif1A activation on cargo binding ................................ 11

Figure 1.4 "Sushi-Model" or "Conveyer-belt" model for efficient delivery of dense core vesicles at synapses.

Figure 1.5 Signaling cascade for activating JNK, a member of mitogen activated protein kinases (MAPK) family

Figure 3.1 Syt4 is localized to DCVs 40

Figure 3.2 DCVs undergo long-range trafficking on microtubules in axon. 42

Figure 3.3 Comparison of synapses at DIV 6 and DIV 13

Figure 3.4 Phosphorylating Syt4 at S135 increases DCV mobility before synapse formation at

DIV6 45

Figure 3.5 Synapse maturation changes DCVs mobility

Figure 3.6 Syt4 vesicles are highly mobile in axons at DIV13. S135E vesicles are least mobile with decreased speed and displacement/s compared to control and S135A vesicles..... 50

Figure 3.7 Syt4 vesicles are less mobile in dendrites at DIV13 .............................................. 52

Figure 3.8 Syt4 S135E shows less interaction with Kif1A motor protein ................................ 53

Figure 3.9 Over-expressing Kif1A rescues S135E vesicle mobility ........................................ 55

Figure 3.10 Syt4 S135E vesicles are captured at pre-synaptic sites...................................... 57

Figure 3.11 Actin disruption leads to more mobility of control and S135E vesicles without changing the trafficking parameters of S135A.

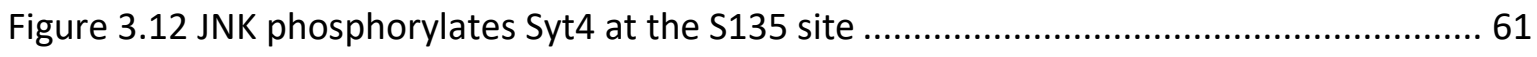

Figure 3.13 Anisomycin and SP600125 effect on trafficking of DCVs .....................................63

Figure 3.14 Phosphorylation of Syt4 at S135 by JNK decreases DCVs mobility and localises these vesicles to synapses 65

Figure 3.15 Activity dependent phosphorylation causes transiting vesicles to pause. ............67

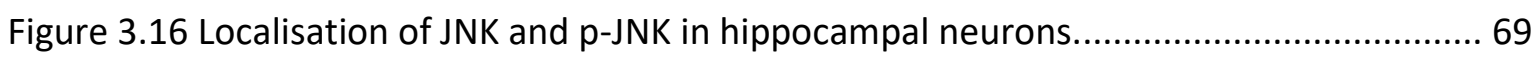

Figure 3.17 Long term stimulation causes more p-JNK and Syt4 at synapses. ........................ 71

Figure 3.18 Fusion of phosphomutants S135A and S135E was similar at both synaptic and non-synaptic sites in hippocampal neurons

Figure 4.1 Proposed model for the capture of dense core vesicles by JNK dependent phosphorylation of Syt4 at S135 at active synapses in hippocampal neurons 


\section{List of tables}

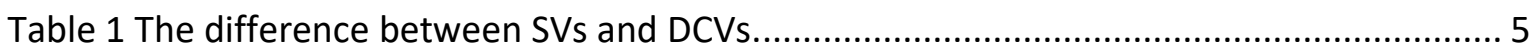

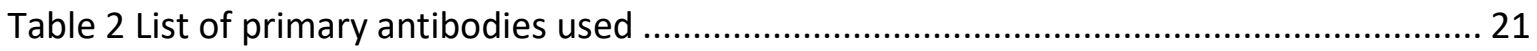

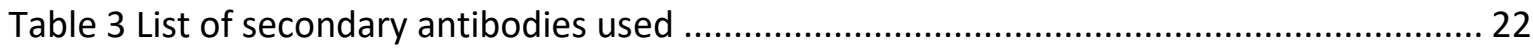

Table 4 List of all plasmids used in this study for over-expression .......................................... 22

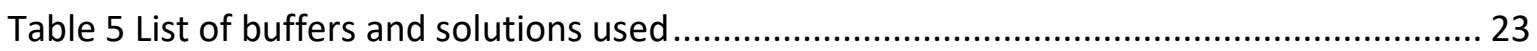

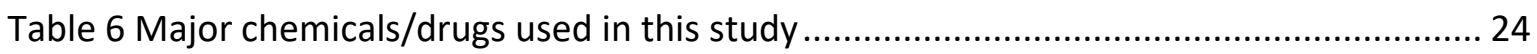





\section{Introduction}

\section{1 Neurons - "building blocks" of the nervous system}

The study of the nervous system encompasses multiple levels ranging from cellular and molecular levels to systems and cognitive aspects. A nervous system is an assembly of neurons connected to each other performing different functions in a regulated and coordinated way. Neurons are the core component of the nervous system and of the brain in particular. They are electrically excitable cells that process and transmit information by electrochemical signalling. A typical neuron possesses three major compartments - a cell soma (the bulbous cell body which contains the cell nucleus, protein synthesis machinery and other organelles), dendrites (long, filaments attached to the cell body in a complex branching "dendritic tree") and an axon (a single specialized, extra-long, branched cellular filament that acts as the output of the neuron) (Jessell and Kandel, 1993).

Every neuron maintains a voltage gradient across its membrane, due to metabolically driven differences in sodium, potassium, chloride and calcium ions within the cell, each of which has a different charge. An electrochemical pulse called the action potential is generated in response to a significant change in the voltage across the membrane (Hodgkin and Huxley, 1952; Jessell and Kandel, 1993). This pulse travels rapidly along the cell's axon to specialized connections known as synapses.

\section{2 Synapse- the "gap" that connects two neurons}

Neurons communicate with each other through a unique structure called the synapse. Synapses are fundamentally classified into electrical or chemical sub-types (Pereda, 2014). Electrical synapses consist of gap junctions that connect the pre- and postsynaptic membrane. Gap junctions contain precisely aligned ionic channels that span synaptic cleft between two neurons. Signal is sent in the form of voltage changes across these gap junctions, which are bi-directional in nature. The main advantage of an electrical synapse is the rapid transfer of signals from one cell to the next (Hormuzdi et al., 2004; Pereda, 2014). 
In a chemical synapse, the pre-synaptic site contains neurosecretory vesicles harbouring chemical messengers called neurotransmitters or neuromodulators. During synaptic neurotransmission, an action potential reaches the pre-synaptic bouton (club-shaped enlargements present in axons) resulting in the opening of voltage-gated $\mathrm{Ca} 2+$ channels. This allows Ca2+ influx, which triggers the release of cargoes from neurotransmittercontaining vesicles into the synaptic cleft. These cargoes bind to their respective receptors on the post-synaptic side of the synapse, which enables transmission of signal between neurons (Jessell and Kandel, 1993; Murthy and De Camilli, 2003)

Both the presynaptic and postsynaptic sites contain extensive arrays of molecular machinery that link the two plasma membranes together and carry out signalling between them (Burns and Augustine, 1995; Murthy and De Camilli, 2003). Although axon-dendrite synaptic connections are the norm, other variations like dendrite-dendrite, axon- axon and axon-soma are also possible.

\section{3 Neurosecretory vesicles - "vehicles" for neurotransmission}

There are two major types of neurosecretory vesicles present in chemical synapses: synaptic vesicles (SVs) and dense core vesicles (DCVs), which store and release distinct cargoes upon stimulations. These two types of neurosecretory vesicles co-exist (Fig 1.1) and perform different functions in neurons (De Camilli and Jahn, 1990; Kelly, 1991; Scalettar, 2006; Gondre-Lewis et al., 2012). While release of cargo from SVs is important for classical neurotransmission across the synapse, DCVs modulate this transmission by releasing cargoes that either increase or decrease neurotransmission. Thus, the coexistence of SVs and DCVs is required for both robust neurotransmission and its modulation, which is essential for regulating various neuronal functions and survival.

\subsubsection{Synaptic vesicles (SVs)}

Found specifically in neurons, synaptic vesicles (SVs) are homogenous circular vesicles with a diameter of $\sim 40-50 \mathrm{~nm}$. These vesicles store and release classical neurotransmitters classified as amino acids including glutamate, g-aminobutyric acid (GABA), and acetylcholine $(\mathrm{ACh})$, or monoamines including dopamine, serotonin, epinephrine, and norepinephrine (Naito and Ueda, 1985; Fykse and Fonnum, 1988; Maycox et al., 1988; De Camilli and Jahn, 1990; Gondre-Lewis et al., 2012). The function of the nervous system 
relies on the release of NTs from these vesicles repeatedly and reliably with every action potential.
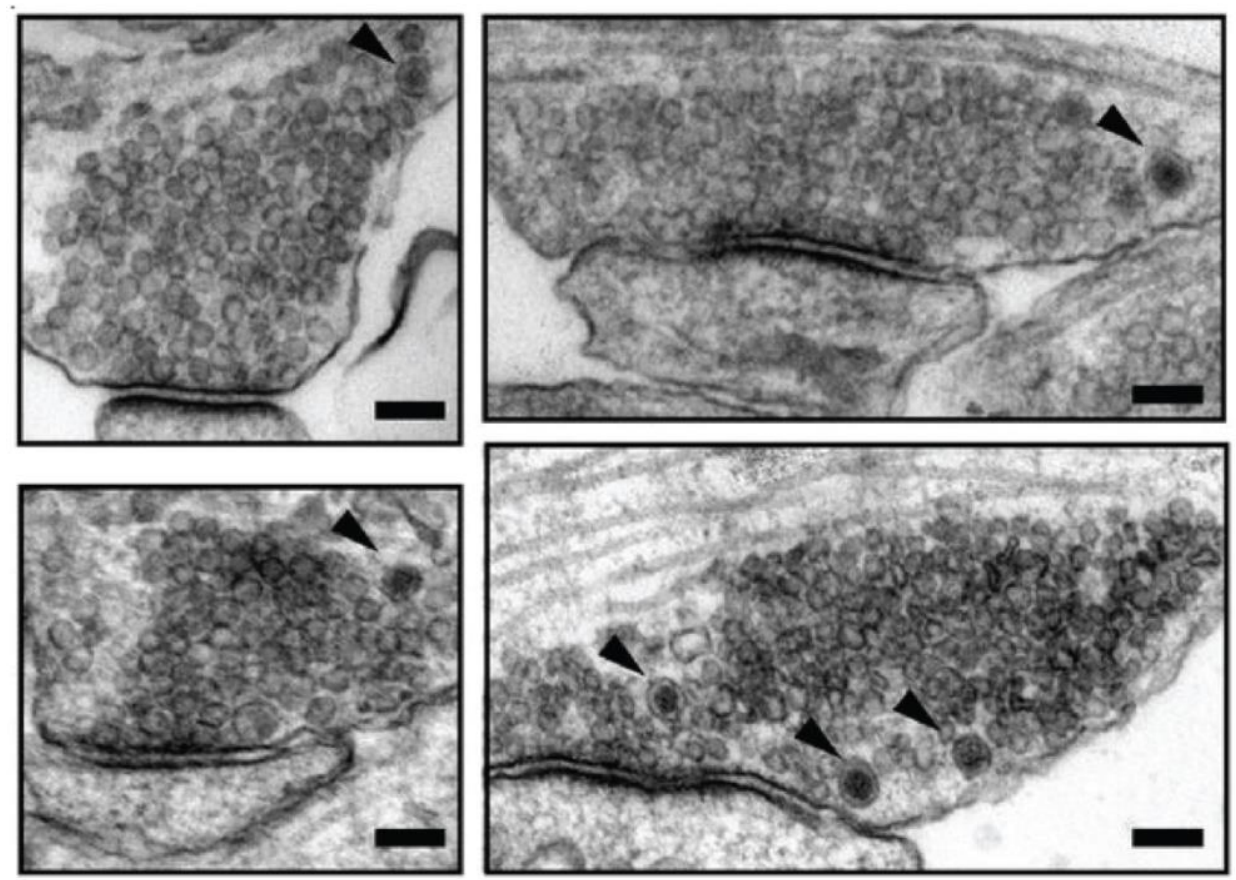

Figure 1.1 Co-existence of SVs and DCVs in the chemical synapse.

Electron micrograph of different synaptic terminals from DIV 14-16 hippocampal neurons harbouring both SVs and DCVs. Black pointed arrows indicate dense core vesicles and small clear core synaptic vesicles fill the pre-synaptic terminal. Scale bar $=100 \mathrm{~nm}$. (van de Bospoort et al., 2012).

\subsubsection{Synaptic vesicle cycle}

The number of vesicles that fuse and their properties of fusion at pre-synaptic sites determines the efficacy and sustainability of signal transduction between neurons (Kononenko and Haucke, 2015). To fulfil the demand and support rapid and repeated rounds of release to continually supply neurotransmitter-filled vesicles to the pre-synaptic bouton, SVs are replenished via a highly regulated trafficking sequence called the "synaptic vesicle cycle" (Heuser and Reese, 1973; Rizzoli, 2014).

Before synaptic vesicles enter into this cycle, the components of SVs are transported from the cell soma, where the majority of proteins are synthesized, to the synapse (Taylor et al., 2013; Rizzoli, 2014). Once SV precursors reach the pre-synaptic site, they enter the "synaptic vesicle cycle" to reform SVs locally within the bouton. The steps involved in this cycle are as follows: 1) SVs are filled with NTs by active transport of neurotransmitter transporters (for example, vGluT for glutamate transport and vGAT for GABA transport) and a vacuolar-type proton pump ATPase that provides a $\mathrm{pH}$ and electrochemical gradient, 
2) Filled SVs are then brought closer to active zones, which are specialized regions of presynaptic plasma membrane containing molecular machinery important for SV recruitment and recycling (Jin and Garner, 2008; Owald and Sigrist, 2009; Sudhof, 2012), 3) SVs dock at the active zone and, 4) are primed by an ATP-dependent process that enables these vesicles to respond to a $\mathrm{Ca}^{2+}$ signal, 5) When an action potential depolarizes the pre-synaptic membrane, $\mathrm{Ca}^{2+}$ influx through voltage-gated $\mathrm{Ca}^{2+}$ channels triggers exocytosis of $\mathrm{SV}^{2}$. $\mathrm{Ca}^{2+}$ dependent exocytosis involves sensing of $\mathrm{Ca}^{2+}$ by Synaptotagmin 1, an integral membrane protein on SVs. Synaptotagmin 1 binds the soluble NSF-attachment protein receptor (SNARE) proteins synaptobrevin on SVs, and SNAP-25 and syntaxin on the pre-synaptic plasma membrane in a $\mathrm{Ca}^{2+-}$ dependent manner, which promotes SV fusion and release of neurotransmitter, 6) After exocytosis, SV membranes are retrieved by endocytosis; there are various endocytic pathways that retrieve SV components, including clathrin-mediated endocytosis, kiss and run, ultrafast and bulk endocytosis (Jung and Haucke, 2007; Watanabe et al., 2013; Wu et al., 2014; Kononenko and Haucke, 2015), 7) SVs are reformed by re-acidifying and refilling of these endocytosed vesicles with NTs either directly or after passing through an endosomal intermediate (Sudhof, 2004, 2012; Kononenko and Haucke, 2015). This local synaptic vesicle cycle at the synapse ensures that the demand and supply of neurotransmitter release from the pre-synaptic terminal is met, leading to efficient neurotransmission.

\subsubsection{Dense core vesicles (DCVs)}

While much has been investigated about SVs, very little is known about DCVs in neurons by comparison. Several studies have focused on neuroendocrine cells to investigate DCVs because of their abundance in these cells. But the regulation of DCV synthesis, transport and availability to release sites in neurons has many open questions.

Dense core vesicles or granules (DCVs) are prominently present in neurons as well as neuroendocrine tissues like pituitary, adrenal glands, endocrine pancreas and gonads (Gorr et al., 2001; Gondre-Lewis et al., 2012). DCVs vary from 100-300 nm in diameter and are more heterogeneous than SVs. They transport, store and release various neuropeptides and neurotrophins like brain derived neurotrophic factor (BDNF), Neurotrophin-3, Neurotrophin-4/5, and Nerve Growth Factor (NGF) (Bean et al., 1994; Poo, 2001; Lessmann et al., 2003; Dieni et al., 2012). While SV fusion results in regular neurotransmission across 
the synapse, release from DCVs modulates that transmission. This neuro-modulation facilitates a spectrum of processes including neuronal survival and differentiation, synaptic plasticity and higher cognitive functions. DCVs are synthesized in the cell body of neurons and are then transported to release sites for fusion (Burgoyne and Morgan, 2003; Kim et al., 2006; Wong et al., 2012). The major differences in SVs and DCVs in neurons are highlighted in the following Table 1.

Table 1 The difference between SVs and DCVs.

\begin{tabular}{|c|c|c|}
\hline Properties & Synaptic Vesicles (SVs) & Dense core vesicles (DCVs) \\
\hline Appearance & $\begin{array}{l}\text { Small, clear, } \\
\text { homogenous, diameter: } \\
\sim 40-50 \mathrm{~nm}\end{array}$ & $\begin{array}{l}\text { Large, dense core, } \\
\text { heterogeneous, diameter: } \\
100-300 \mathrm{~nm}\end{array}$ \\
\hline $\begin{array}{l}\text { Numbers at synaptic } \\
\text { boutons }\end{array}$ & $\begin{array}{l}\text { Highly enriched in } \\
\text { synaptic terminals. 100- } \\
200 \text { vesicles at a single } \\
\text { pre-synaptic site (Ikeda } \\
\text { and Bekkers, 2009; Alabi } \\
\text { and Tsien, 2012) }\end{array}$ & $\begin{array}{l}\text { Number ranges from 1-10 at } \\
\text { pre-synaptic sites (Sorra et } \\
\text { al., 2006) }\end{array}$ \\
\hline Cargoes & $\begin{array}{l}\text { Neurotransmitters like } \\
\text { Glutamate, GABA, } \\
\text { Acetylcholine, } \\
\text { Dopamine, Serotonin }\end{array}$ & $\begin{array}{l}\text { Neuromodulators like BDNF, } \\
\text { NT-3, NT-4, NGF, } \\
\text { neuropeptide Y (NPY) }\end{array}$ \\
\hline $\begin{array}{l}\text { Assembly and delivery to } \\
\text { release sites }\end{array}$ & $\begin{array}{l}\text { Locally recycled at pre- } \\
\text { synaptic terminals. } \\
\text { Undergo highly } \\
\text { organized "synaptic } \\
\text { vesicle cycle". }\end{array}$ & $\begin{array}{l}\text { Synthesized in cell body and } \\
\text { are then transported along } \\
\text { microtubules to release } \\
\text { sites. }\end{array}$ \\
\hline$\underline{\text { Stimuli causing release }}$ & $\begin{array}{l}\text { Lower frequency stimuli } \\
\text { are sufficient to induce } \\
\text { release. Duration of } \\
\text { cargo release is short } \\
\text { (ms). }\end{array}$ & $\begin{array}{l}\text { High frequency stimuli are } \\
\text { required for release. } \\
\text { Duration of cargo release } \\
\text { can be long (seconds to } \\
\text { minutes). }\end{array}$ \\
\hline
\end{tabular}




\begin{tabular}{|l|l|l|}
\hline Functions & $\begin{array}{l}\text { Core component of } \\
\text { classical synaptic } \\
\text { neurotransmission. }\end{array}$ & $\begin{array}{l}\text { Modulates classical synaptic } \\
\text { transmission by SVs. }\end{array}$ \\
\hline
\end{tabular}

\section{4 Biogenesis of DCVs}

There are various steps involved in forming mature dense core vesicles, which are common to both neurons and neuroendocrine cells (Kim et al., 2006). Unlike SVs, DCVs are generally packed with their cargoes in the cell soma. They do not undergo formation or recycling at the synaptic terminal like SVs. Cargoes likes neuropeptides or neurotrophins are generated as large precursors at the rough endoplasmic reticulum ( $r E R)$ and are then inserted in ER cisternae to be properly folded. Subsequently, they are transported to the Golgi apparatus for further sorting, modification and packaging. In the trans-golgi network, cargoes destined for DCVs are sorted away from other proteins by aggregating at an acidic $\mathrm{pH}$ in the presence of calcium. These aggregated proteins are then engulfed in budding vesicles to form immature dense core vesicles (Orci et al., 1987). The further acidification of vesicles to approx. pH 5.5 and progressive processing of cargoes in the regulated secretory pathway converts these immature vesicles to mature, exocytosis competent vesicles (Borgonovo et al., 2006) (Gondre-Lewis et al., 2012). Mature DCVs synthesized are then transported longdistances to release sites in the axon via cytoskeletal components (Goldstein and Yang, 2000; Rudolf et al., 2001; Rudolf, 2003; Neco et al., 2003; Smith et al., 2003; Guzik and Goldstein, 2004)

\section{5 Intracellular transport of DCVs}

\subsubsection{Cytoskeletal elements}

The neuronal cytoskeleton consists of three major components - actin filaments (F-actin), microtubules and neurofilaments. Septins have been proposed recently to represent a fourth cytoskeleton element (Mostowy and Cossart, 2012). All of these components provide intrinsic structural support and control growth and migration of neurons and neuronal processes (Coles and Bradke, 2015). In axons and dendrites, microtubules are the major longitudinal cytoskeletal filaments whereas actin filaments are enriched in synaptic 
regions at pre-synaptic terminals and post-synaptic spines and as cortical rings that may provide support along the shafts of long neurites (Fig 1.2) (Hirokawa et al., 2010; Zhong et al., 2014).

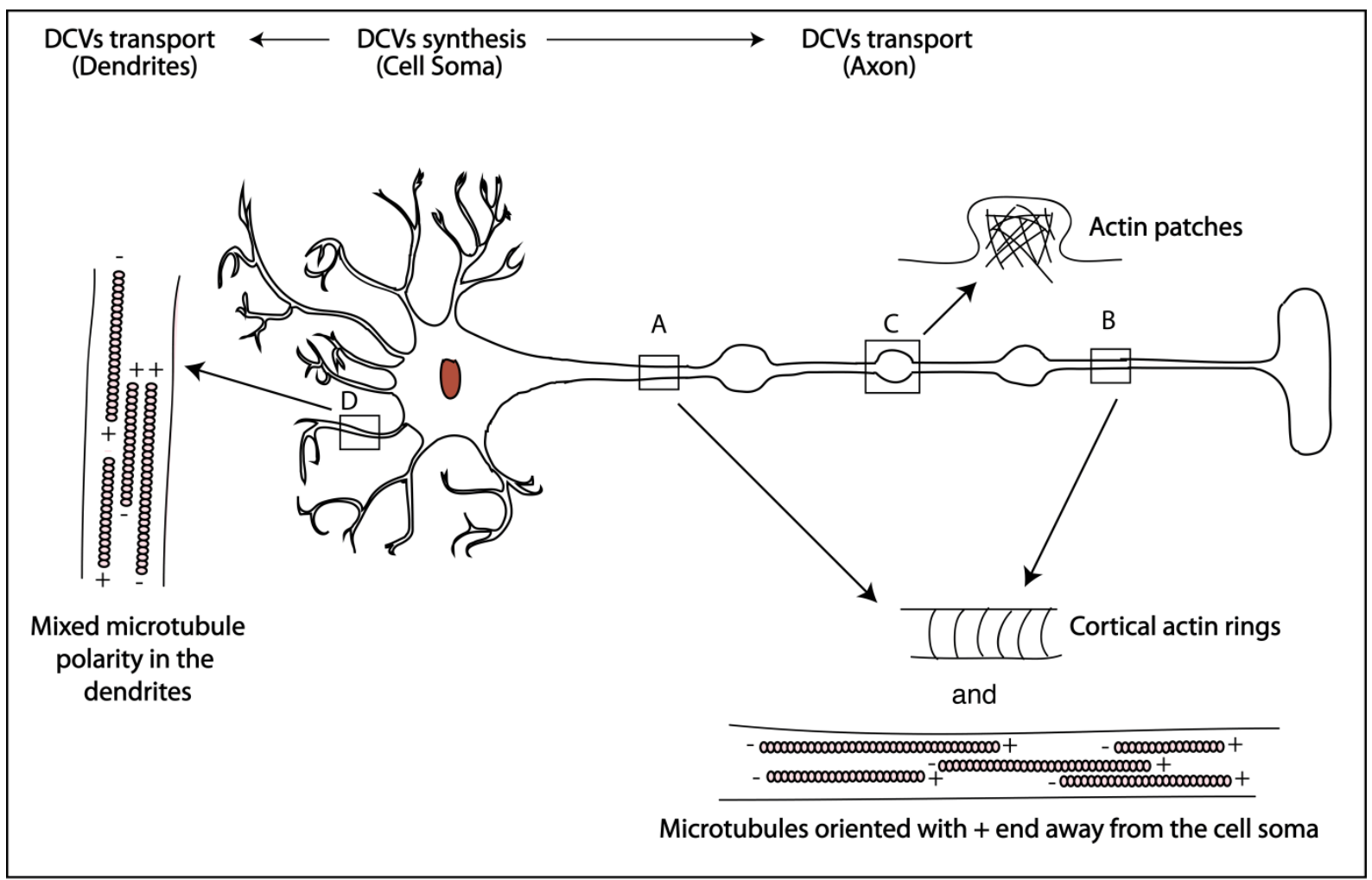

Figure 1.2 Actin and microtubule architecture in a neuron

DCVs are synthesized in the cell soma and are transported in axons and dendrites by cytoskeletal elements. Cartoon represents a mammalian hippocampal neuron in vitro. Boxed and expanded view of the boxes highlights the actin and microtubules architecture in each compartment. (A) and (B) show cortical actin rings and microtubule arrangement in an axon. Cortical actin rings display a periodicity of approximately $190 \mathrm{~nm}$ and may provide support along the shafts of long neurites. Microtubules in axons, on the other hand, serve as long tracks arranged with a single polarity; the + end is always oriented away from the cell soma. (C) Factin is arranged in a meshwork like structure at pre-synaptic sites in an axon. (D) In dendrites, there is mixed polarity of microtubules. (Adapted from (Coles and Bradke, 2015).

Microtubules (MTs) serve as tracks for long-range transport in the neuron (Maeder et al., 2014). MTs are polarized tubulin polymers (protofilaments made up of heterodimers of $\alpha$ tubulin and $\beta$-tubulin arranged in head to tail fashion) with fast growing plus ends and more stable minus ends (Maday et al., 2014). MTs undergo assembly and disassembly (through GTP hydrolysis), also known as dynamic instability, at the plus end, which allows the expansion and retraction of MTs in a cell (Howard and Hyman, 2009; Kapitein and Hoogenraad, 2015). In axons, MTs form a unipolar array with plus end oriented outwards towards the growth cone (Ahmad, 1992; Stepanova et al., 2010) while in dendrites they are 
found in mixed polarity (Baas et al., 1988; Kwan et al., 2008; Kleele et al., 2014). MT based long-range transport is essential for neuronal survival and function both during development and in the adult brain (Franker and Hoogenraad, 2013).

For short range trafficking and local delivery of cargoes to synapses and growth cones, actin filaments are crucial. F-actin assembles from globular actin monomers (G-actin) into a twostranded helical structure. It also possesses an intrinsic polarity by having two distinct ends termed as a barbed or plus end responsible for actin growth and a pointed or minus end for dissociation (Pollard and Cooper, 2009; Kapitein and Hoogenraad, 2011). Actin undergoes ATP hydrolysis upon polymerization. Actin filaments and microtubules together orchestrate cytoskeletal dynamics in neurons (Coles and Bradke, 2015).

Newly synthesized DCVs in the cell soma are trafficked to axons and dendrites along these cytoskeletal components. DCVs are highly mobile and show distinct trafficking properties in axons and dendrites, likely due to differences in cytoskeletal components in these two regions (Overly et al., 1996; de Wit et al., 2006; Kwinter et al., 2009). DCVs travel long distances on MTs and short intra-bouton distances on F-actin with the help of associated motor proteins in axons. In dendrites, DCVs traffic slowly and cover less distance compared to in axons.

\subsubsection{Motor proteins for vesicle trafficking}

The mechanism of delivering specific organelles or vesicles in neurons primarily depends on molecular motor proteins that move along the cytoskeletal elements. The molecular motor kinesin, dynein and myosin super families have been identified to transport various cargoes in the neuron (Hirokawa, 1998; Karki and Holzbaur, 1999; Vallee et al., 2009; Hirokawa et al., 2010). Actin filaments facilitate the motility of myosin family motor proteins. These proteins use ATP hydrolysis energy as the means for generating movement on actin filaments. Among the 35 known myosin proteins, myosin Va and b, myosin II and myosin VI are majorly involved in activity-dependent synaptic trafficking (Kneussel and Wagner, 2013).

Myosin Va stands out among the myosins in the context of dense core vesicles, as it plays important roles in DCV biogenesis, transport along actin filaments and regulation of exocytosis in PC-12 cells, neuroendocrine cells and neurons (Kogel et al., 2010). Myosin Va 
drives directional motility on actin filaments, ensuring the delivery of cargoes at the synaptic terminals (Ali et al., 2007).

Kinesins and dynein move towards the microtubules plus-end or minus-end, respectively, to transport cargoes longitudinally in axons and dendrites (Kardon and Vale, 2009; Hirokawa et al., 2010; Kneussel and Wagner, 2013; Maeder et al., 2014). The kinesin superfamily of 45 genes is subdivided into 14 subfamilies based on the homology of their motor domain, structural and functional similarities out of which kinesin-1, kinesin-2 and kinesin-3 families contribute to axonal transport in neurons (Lawrence et al., 2004; Maday et al., 2014). On the contrary, one single gene encodes the motor subunit of dynein (Roberts et al., 2013). While kinesin controls anterograde movement, cytoplasmic dynein drives retrograde transport.

Dense core vesicles undergo both anterograde and retrograde movement in the neuron. DCVs travel extremely long distances, emphasizing the need of microtubule-based motors kinesins and dyneins (Zahn et al., 2004; Barkus et al., 2008; Kwinter et al., 2009).

\section{6 Kinesin-3 family, Unc-104/Kif1A: a motor protein for DCVs}

The kinesin-3 family is one of the largest among the kinesin superfamily that functions as the "marathon runners of the cellular world" (Soppina et al., 2014). For long range trafficking of dense core vesicles, the kinesin-3 family member Unc-104/Kif1A is required for transport along MTs in both invertebrate and mammalian neurons (Bloom, 2001; Kim et al., 2006; Barkus et al., 2008; Lo et al., 2011; Soppina et al., 2014). Conventional kinesins such as kinesin-1 and most other kinesin superfamily proteins (Kifs) possess a two-headed dimer structure that moves in a "hand over hand" pattern. However, the kinesin-3 family protein KIf1A is a neuron specific monomeric motor protein characterized by a single motor domain, a forkhead-associated domain and a C-terminal lipid-binding domain (pleckstrin homology domain) (Soppina et al., 2014). Thus, the hand over hand model doesn't explain the motility of Kif1A due to the lack of a second hand or natural dimer structure.

Previous studies have reported a diffusive motility of Kif1A by weak electrostatic interaction between its positively charged $\mathrm{K}$-loop and the C-terminal negatively charged $\mathrm{E}$ hook of microtubules (Okada and Hirokawa, 2000). Since then it was shown that Kif1A is regulated by a unique mechanism in which non-cargo bound motors are monomeric and 
inactive whereas cargo-bound Kif1A are dimeric resulting in long processive motion for transport of cargoes at speeds of $\sim 2 \mu \mathrm{m} / \mathrm{sec}$ (Hammond et al., 2009; Soppina et al., 2014). The key mechanism regulating this monomer to dimer transition involves regulating the neck coil (NC) and coiled coil 1 (CC1) region present in front of FHA domain in the Kif1A moiety. In the absence of cargo, an intramolecular NC-CC1 parallel conformation maintains Kif1A in a monomeric inactive state that is removed when cargo binds to the motor. Cargo binding favours an intermolecular NC-NC interaction resulting in dimerization and high processivity of Kif1A. The FHA and CC domains are important for auto-inhibition of Kif1A, which blocks interaction with microtubules (Huo et al., 2012). The C-terminal pH domain is crucial for recognition of phospholipids in cargo vesicle membranes (Fig 1.3) (Klopfenstein et al., 2002; Lee et al., 2003; Al-Bassam et al., 2003). Liprin-alpha or synapse defective 2 (SYD 2) is reported to bind to the tail region of Kif1A and is a multimodular scaffolding protein that acts as a Kif1A receptor on cargo vesicles as well as a stabilizer of Kif1A dimers (Shin et al., 2003).

The high processivity of Kif1A ensures the long-range anterograde trafficking of DCVs from the cell soma to release sites in neurons. Kif1A controls the anterograde trafficking of DCVs both in axons and dendrites in hippocampal neurons. In dendrites, Kif1A shows limited movements compared to the fast and processive movement observed along axonal microtubules (Lee et al., 2003; Shin et al., 2003). 


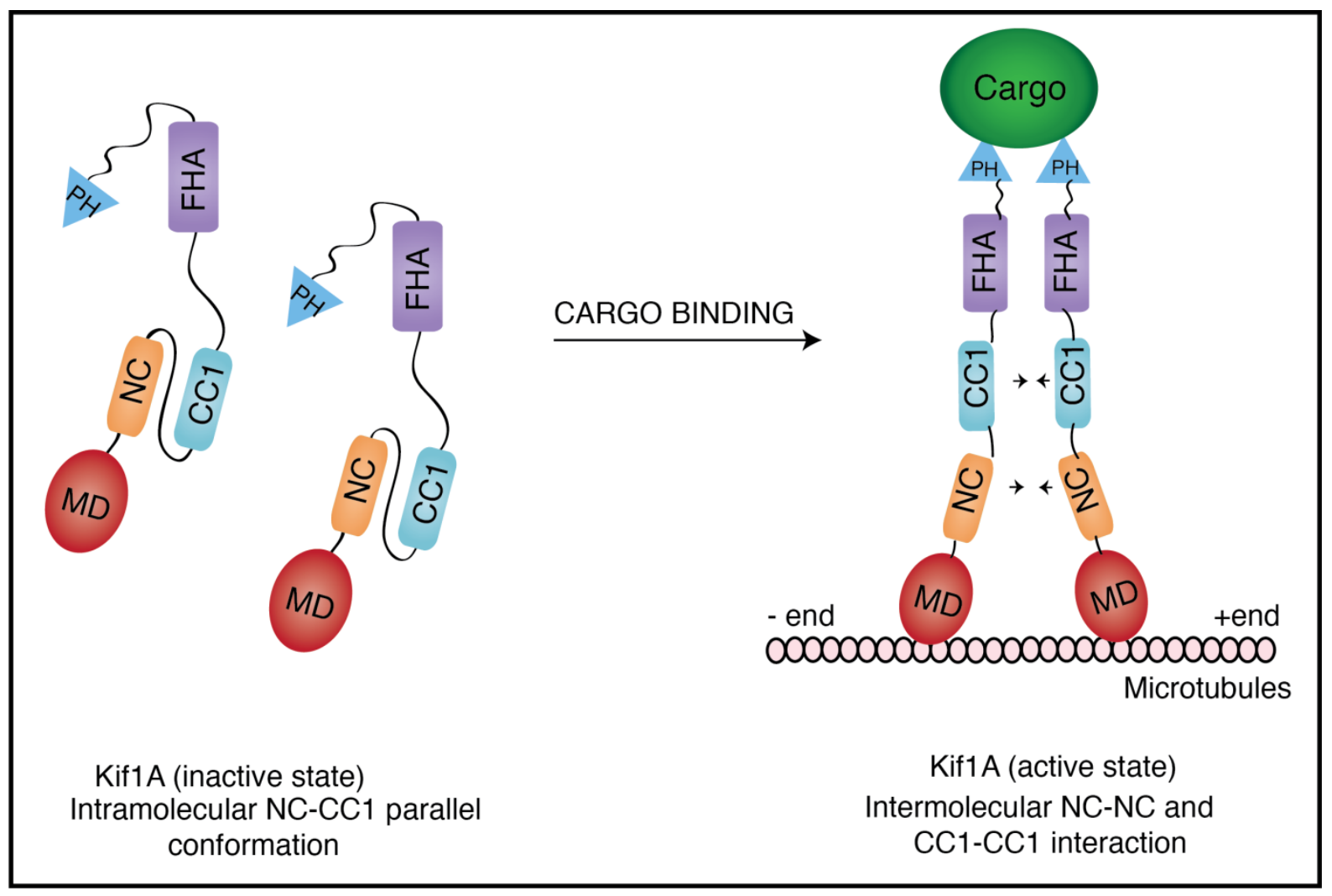

Figure 1.3 Molecular mechanism of Kif1A activation on cargo binding

Kif1A exists in both monomeric and dimeric states. It employs a unique mechanism of regulation by forming dimers upon cargo binding. In the absence of cargo, intramolecular NC-CC1 region of Kif1A is proposed to form a parallel conformation leading to inactive Kif1A. Cargo binding increases the effective concentration of Kif1A because of intermolecular NC-NC and CC1-CC1 interactions leading to dimerization and high processivity on microtubules. MD: motor domain, NC: neck coil region, CC1: Coiled coil region1, FHA: fork head associated and PH: pleckstrin homology. (Adapted from (Soppina et al., 2014)

Apart from DCVs, unc-104/Kif1A is majorly responsible for anterograde transport of synaptic vesicle precursors both in C.elegans and in mammals (Okada et al., 1995; Okada and Hirokawa, 2000) (Hall and Hedgecock, 1991). Defects in the transport regulated by Kif1A lead to impairment in synapse development and accumulation of synaptic precursors in the soma (Yonekawa et al., 1998; Maday et al., 2014). Conversely, overexpression of Kif1A promotes synapse formation (Kondo et al., 2012).

\section{7 "Sushi-model" for dense core vesicle transport}

The one-way model of anterogradely transporting cargo-filled dense core vesicles from the cell soma to release sites doesn't fulfil the possibility of equal distribution of vesicles to all en passant boutons in neurons. Previous work in the Drosophila neuromuscular junction has shown that DCVs follow the "sushi-model" or "conveyer belt model" of DCV transport 
in which there is a continuous circuitous trafficking of DCVs throughout the axon driven by molecular motors just like a conveyer belt in a sushi restaurant (Fig 1.4). Transiting DCVs from the cell soma are routed first to go to the most distal bouton where they switch directionality to move retrogradely and then fulfil the demand of proximal boutons (Wong et al., 2012; Moughamian and Holzbaur, 2012).

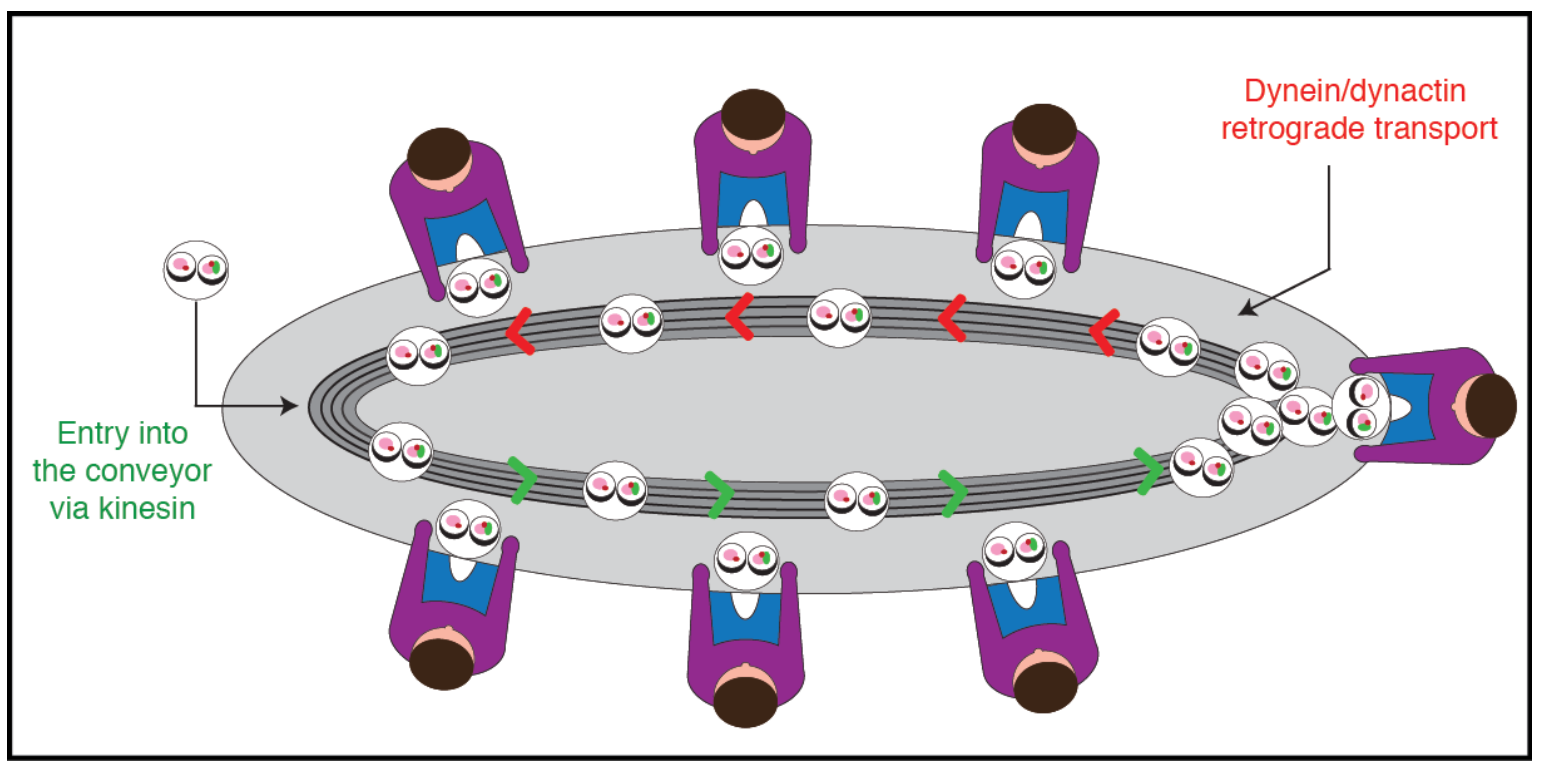

Figure 1.4 "Sushi-Model" or "Conveyer-belt" model for efficient delivery of dense core vesicles at synapses

Dense core vesicle delivery to release sites is analogous to people at a conveyer belt in a sushi restaurant. Each plate of sushi is like a DCV with cargo and patrons are synapses. As the sushi travels around the conveyer belt and patrons take plates from the belt whenever they want, similarly, dense core vesicles traffic in neuron in a circuitous manner and synapses take DCVs from the transiting pool whenever required. As the conveyer belt model fulfils the demand of every patron sitting around the table equally, in the same fashion, dense core vesicles demand of all synapses are fulfilled (Adapted from (Moughamian and Holzbaur, 2012) with permission from publisher) Elsevier License number 3810860661881.

This circuitous trafficking pattern of DCVs from the soma to distal axonal sites and back again ensures the continuous trafficking of cargo-filled vesicles in axons. These transiting DCVs are captured at synapses in an activity-dependent manner, where the efficiency of capture determines the size of the accessible pool of vesicles at individual sites (Shakiryanova et al., 2006; Wong et al., 2012; Bulgari et al., 2014). The probability that transiting vesicles are captured depends on the demand of the release site. However, whether the circuitous trafficking of DCVs is a general phenomenon that also occurs in mammalian neurons to ensure the uniform presynaptic distribution of neuropeptide stores is still unknown. 


\section{8 Synaptotagmin 4: a membrane protein of DCVs}

The synaptotagmin (Syt) protein family consists of 17 members that play an important role in regulating a variety of membrane trafficking events in the cell. Many Syts share a common structure consisting of five major domains: an intravesicular $\mathrm{NH} 2$-terminal domain, a single trans-membrane domain, a spacer domain and two homologous C2 domains ( $\mathrm{C} 2 \mathrm{~A}$ and $\mathrm{C} 2 \mathrm{~B}$ ) located in the cytoplasmic $\mathrm{COOH}$-terminal region (Yoshihara and Montana, 2004; Bhalla et al., 2008; Mori and Fukuda, 2011). C2 domains in most Syts act as calcium sensors which promote membrane fusion by penetrating lipid bilayers and binding to soluble NSF attachment protein receptors (SNAREs) in a $\mathrm{Ca}^{2+}$ dependent manner (Nalefski and Falke, 1996). Notably, there is a difference in calcium sensitivity and kinetics of Syt interactions among different Syts.

Synaptotagmin 4 (Syt4), a brain-specific $47 \mathrm{kDa}$ Syt, harbours a conserved aspartate to serine substitution in the $\mathrm{C} 2 \mathrm{~A}$ domain leading to decreased $\mathrm{Ca}^{2+}$ dependent phospholipid binding activity, with the exception of Drosophila Syt4 (Ullrich et al., 1994; von Poser et al., 1997). Because of this unique feature in Syt4, its role as a positive or negative regulator in vesicle fusion is a matter of debate. Several studies have indicated that Syt4 doesn't act as a pre-synaptic $\mathrm{Ca}^{2+}$ sensor like Syt1, but rather works as a post-synaptic or glial $\mathrm{Ca}^{2+}$ sensor (Ibata et al., 2002; Zhang et al., 2004; Yoshihara and Montana, 2004). Syt4 has been implicated in increasing; decreasing or modulating $\mathrm{Ca}^{2+}$ evoked release in a context and cell type dependent manner (Thomas et al., 1999; Eaton et al., 2000; Wang et al., 2001; Wang et al., 2003; Tsuboi and Rutter, 2003; Zhang et al., 2004; Machado et al., 2004). Syt4 mRNA is expressed in all brain regions but mainly in the hippocampus, cortex and cerebellum (Berton et al., 1997). The subcellular localization of Syt4 was debated for a long time in differing reports. It was shown to be prominent in Golgi in the cell soma and neurites (Ibata et al., 2000), and on DCVs in PC-12 cells (Osborne et al., 1999; Ibata et al., 2002; Ting et al., 2006). Its localization to SVs in neurons was reported (Littleton et al., 1999; Berton et al., 2000), and later it was reported to be absent from SVs and post-synaptic at the NMJ (Yoshihara et al. Science 2005). Recently, Syt4 was shown to localize to BDNF-containing DCVs in both axons and dendrites of hippocampal neurons (Dean et al., 2009). Furthermore, it was shown to negatively regulate BDNF release leading to changes in synaptic function and plasticity in neurons (Dean et al., 2009). Syt4 is also particularly 
interesting as it is an immediate early gene upregulated by seizures and activity (Vician et al., 1995; Mori and Fukuda, 2011). In humans, it maps to a region of chromosome 18 associated with human psychiatric disorders, schizophrenia and bipolar diseases (Ferguson et al., 2000; Ferguson et al., 2004).

Interestingly, the S135 site of Syt4 located in the spacer region between the transmembrane domain and the C2A domain, is phosphorylated by the protein kinase JNK (c-Jun N-terminal kinase) both in vitro and in vivo (Mori et al., 2008). JNK-dependent phosphorylation of Syt4 at S135 is important for translocating Syt4 from immature to mature secretory vesicles and also increases $\mathrm{Ca}^{2+}$ evoked release in NGF treated PC-12 cells (Mori et al., 2008).

\section{9 JNK and its role in axonal transport}

JNK, also known as stress-activated protein kinase (SAPK), is a proline-directed protein kinase that phosphorylates serine or threonine residues. It comprises of ten splice variants derived from three genes: JNK1 (four isoforms), JNK2 (four isoforms) and JNK3 (two isoforms) that are all either $46 \mathrm{kDa}$ or $54 \mathrm{kDa}$ in size (Kyriakis and Avruch, 2012). However, they differ in expression in the mammalian brain (Lee et al., 1999; Brecht et al., 2005). JNK1 and JNK3 subcellular distributions differ in the cortex, hippocampus and cerebellum. JNK3 is found in $90 \%$ of pyramidal layer neurons whereas JNK1 is majorly seen in CA3, CA4 and the hilus of the dentate gyrus in the hippocampus (Lee et al., 1999).

JNK belongs to the classical mitogen activated protein kinase (MAPK) signalling cascade. In this cascade, MAPK (JNK and p38) are activated by dual phosphorylation of the threonineproline-tyrosine (TPY) motif of upstream MAP kinase kinase (MAPKK) (MKK4 and MKK7 for JNK and MKK3 or MKK6 for p38). These MAPKK are themselves activated by a diverse group of MAP kinase kinase kinases (MAPKKK). The MAPK pathway is a three-tiered signalling cascade, which is activated by various stimuli including stress signals, growth factors, hormones, and cytokines (Fig 1.5) (Davis, 2000).

JNK is amongst the most abundant kinases in the brain (Whalley, 2008) and is important for controlling stress responses in addition to normal physiological processes (Ip and Davis, 1998; Davis, 2000). 
STIMULI Stress, Cytokines, growth hormones etc.

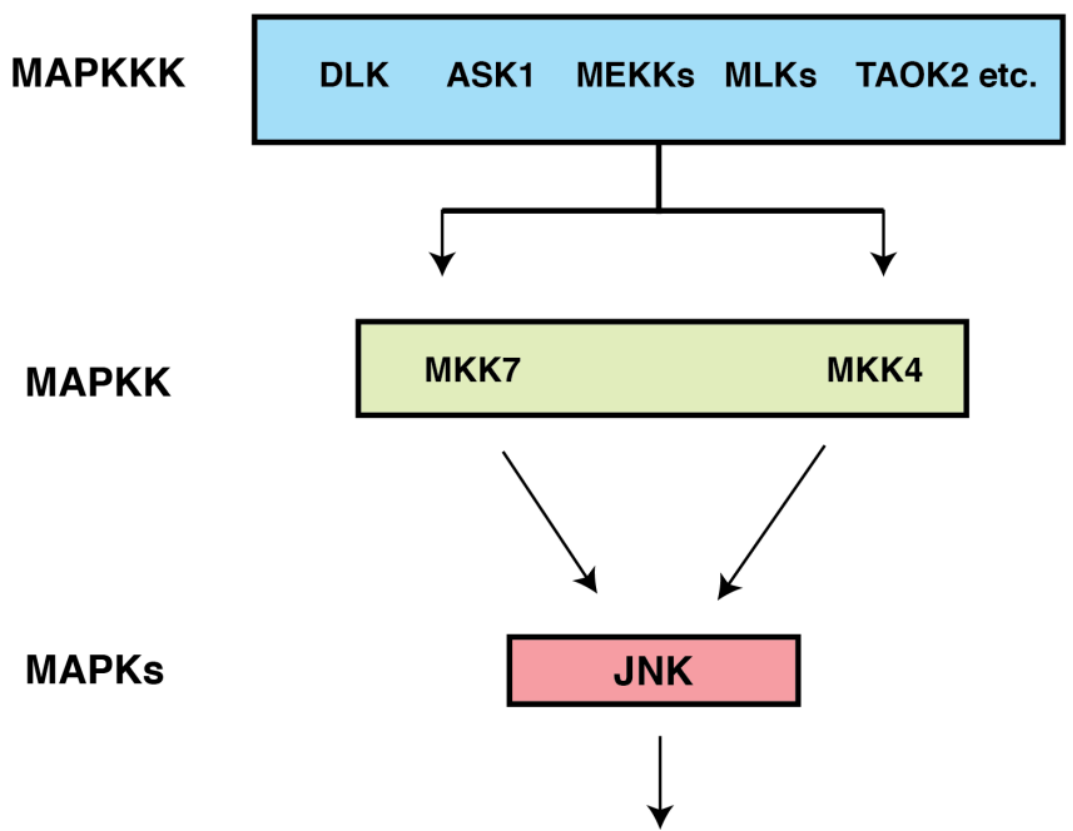

Nuclear and/or cytoplasmic substrates

Figure 1.5 Signaling cascade for activating JNK, a member of mitogen activated protein kinases (MAPK) family

The MAPK pathway is a three-tiered signalling cascade in which JNK (c-Jun N-terminal kinase) is activated by dual phosphorylation of threonine and tyrosine in the TPY motif by two MAPK kinases, MKK7 and MKK4. The MAPKK members are activated, in turn, by phosphorylation by a group of MAPKKKs. The whole signalling cascade is activated in response to stimuli like stress, cytokines, and growth hormones. Activation of JNK in this cascade finally phosphorylates several nuclear and/or cytoplasmic substrates, which play various important roles in the cell. MAPK: mitogen activated protein kinase, ASK1: apoptosis signal regulating kinase1, DLK: dual leucine zipper bearing kinase, MEKKs and MLKs: MAPKK kinases, TAOK2: thousand-and-one amino acid kinase 2 (Adapted from (Davis, 2000; Coffey, 2014) Permissions taken from Nature publishing group. License number 3810870567210.

Apart from being well known for its role in neuronal apoptosis, it also promotes neuronal differentiation and migration, synaptic plasticity and memory formation (Behrens et al., 1999; Oliva et al., 2006; Westerlund et al., 2011; Coffey, 2014). In addition, JNK has been reported to be involved in long range vesicle trafficking to support neuronal functions. It regulates axonal transport by phosphorylating motor and adapter proteins and cargoes. For example, JNK3 phosphorylates the motor protein Kif5C at S176; reducing kinesin-1 interaction with microtubules and in turn inhibiting fast anterograde axonal transport, in squid axoplasm (Morfini et al., 2006; Morfini et al., 2009). JNK also controls the directionality of transport via phosphorylation of adapter proteins for both kinesins and dyneins, such as JNK-interacting protein (JIP1). Phosphorylation of JIP1 at S421 promotes 
anterograde axonal transport by kinesin-1 and non-phosphorylated JIP1 favours retrograde axonal transport of amyloid precursor protein (APP) in rodent neurons (Fu and Holzbaur, 2013). Unloading of kinesin-transported cargoes from tubulin tracks has also been reported to involve the JNK pathway in neurons (Stagi et al., 2006; Thomas et al., 2009; Wu et al., 2013; Gibbs et al., 2015).

As robust axonal transport is key for neuronal function and development, deregulation of axonal transport by protein kinases has been implicated in several neurological disorders including Alzheimer's disease, Amyotrophic Lateral Sclerosis, and Huntington's disease. In Huntington's disease, JNK3 is activated by mutant huntingtin (polyQ-htt) resulting in phosphorylation of the kinesin-1 motor domain. Phosphorylation of kinesin-1 causes dissociation of motor proteins from microtubules leading to impaired axonal transport (Morfini et al., 2009).

\section{10 Capture of transiting DCVs}

The efficient circuitous trafficking of DCVs is important to supply cargo filled vesicles from the cell soma to en passant boutons; the heterogeneity in DCV stores at these synapses indicates the importance of vesicle capture from the transiting pool. It was long believed that the cell soma controls the delivery of DCVs at various release sites and that these release sites just wait passively to receive cargo. However, this model is incapable of delivering cargoes specifically to active synapses and doesn't support different neuropeptidergic stores at different synapses. In addition, this system would involve significant delays in cargo traffic from the cell soma to distal boutons in neurons; especially given that some sensory and motor neurons have terminals $>1 \mathrm{~m}$ away from the cell soma.

Efficient vesicle capture by synapses is likely important to maintain and modulate neurotransmission quickly. In Drosophila, DCV capture has been shown to be under transcriptional control influencing neuron-specific variation in peptidergic function (Bulgari et al., 2014). In addition, the same group provided evidence that difference in DCV number at synapses is not due to differences in delivery from the cell soma but is a result of presynaptic capture. DCVs move in both anterograde and retrograde directions to supply vesicles and these transiting vesicles are captured bi-directionally in axons (Wong et al., 2012; Wong et al., 2015). 
The specificity of certain synapse to have more DCVs compared to others is conferred by the activity dependence of vesicle capture. Activity-dependent capture of vesicles at synapses provides a mechanism to fulfil the demand of even distally located nerve terminals (Shakiryanova et al., 2006; Wong et al., 2015) in a synapse-specific manner. Another remarkable property of activity-dependent capture of DCVs is that the process itself is reversible and repeatable (Shakiryanova et al., 2006). The reversibility of the capture process indicates that captured vesicles have the ability to return to transiting pool and the repeatable nature of capture suggests that DCVs can fuse multiple times at presynaptic sites (Shakiryanova et al., 2006; Wong et al., 2012).

\section{11 Fusion and release of cargo from DCVs}

Unlike SVs, which recycle locally at synapses, dense core vesicles undergo long-range trafficking, are captured from the transiting pool of vesicles and undergo fusion whenever required. Release of cargo from DCVs requires typically high frequency stimuli compared to that required for SVs fusion (Scalettar, 2006).

DCVs generally are seen in $20-30 \%$ of axonal boutons in mature hippocampal neurons; ranging 1-10 in absolute number. They are usually located away from the active zone, and hence might require intra-bouton trafficking on actin filaments before being docked and tethered at synaptic sites (Sorra et al., 2006). DCV cargo can also be released from nonsynaptic/extra-synaptic sites, but upon more prolonged stimulation compared to synaptic DCVs (van de Bospoort et al., 2012; Trueta and De-Miguel, 2012). Several studies have shown various types of DCVs fusion, ranging from full fusion resulting in complete release of cargo, to kiss-and-run fusion leading to partial release of cargo, and "crash fusion" - an accelerated fusion that doesn't involve the classical linear pathway of steps requiring docking-tethering and subsequent fusion of vesicles with the plasma membrane (Verhage and Sorensen, 2008; Xia et al., 2009; Matsuda et al., 2009; Wu et al., 2014).

While much is known about the synaptic vesicle fusion machinery, very little is known about DCV fusion machinery in neurons. Though the transport and exocytotic mechanisms of SVs are different compared to DCVs, these two vesicle types still share common properties like the need for functional SNARE proteins and calcium influx through voltagegated calcium channels to promote fusion ( $X u$ and $X u, 2008)$.Munc13-1/2 are essential priming proteins involved in SV exocytosis (Burns and Augustine, 1995; Varoqueaux et al., 
2002; Rhee et al., 2002; Basu et al., 2007) whereas for DCVs, these proteins help in priming but are not critical for release. Knockouts of these proteins reduce synaptic DCV release, and in its absence prolonged stimulation is required at synapses to release remaining vesicles (van de Bospoort et al., 2012). Calcium activator protein for secretion-1 (CAPS-1) is another essential protein reported for priming of DCVs for fusion at both synaptic and non-synaptic sites. CAPS-1 deletion has been shown to impair fusion competence of stationary DCVs in pre-synaptic terminals of mammalian neurons (Farina et al., 2015). Another important class of proteins for vesicle fusion are synaptotagmins which act as $\mathrm{Ca}^{2+}$ sensors and facilitate fusion of vesicles with the target plasma membrane (Sudhof, 2002). Syt1 is a well-characterized protein present on SVs responsible for their fusion and neurotransmission, but it is not clear which Syt isoform (or other calcium-sensing protein) is responsible for DCV fusion in neurons. In PC-12 cells, DCVs are very heterogeneous, and different Syt isoforms, including Syt 1, 4, 7 and 9 were shown to regulate the exocytosis of DCVs depending on the type of fusion (Zhang et al., 2011). By using pHluorins (pH-sensitive markers whose fluorescence is quenched in acidic environments and dequenched/visible in a basic environment), it was demonstrated that even in hippocampal neurons, there might be multiple Syts isoforms responsible for DCV fusion (Dean et al., 2012). Syt4pHluorin present on BDNF-containing dense core vesicles shows distinct kinetics compared to Syt1-phluorin in hippocampal neurons (Dean et al., 2009). A pHluorin attached to Syt1 on the luminal side of SVs exhibits a fast increase in fluorescence on depolarization (corresponding to exocytosis), followed by a steep decline within 60 - $90 \mathrm{~s}$ indicating endocytosis and reacidification of synaptic vesicles (Miesenbock et al., 1998; Sankaranarayanan and Ryan, 2000; Dean et al., 2012). On the contrary, Syt4-pHluorin shows a slower rise followed by prolonged elevation of fluorescence for several minutes upon depolarization further indicating its distinct localization to DCVs. The majority of other Syts show DCV-like fusion properties in hippocampal neurons, suggesting that multiple Syt isoforms may regulate DCV fusion on similar or distinct vesicle subpopulations. 


\section{12 Aim and scope of thesis}

As mentioned above, neuronal dense core vesicles (DCVs) facilitate many crucial functions including neuronal differentiation, migration and plasticity by transporting and releasing neurotrophins and neuropeptides at sites where they are needed (Scalettar, 2006; Wong et al., 2012). Unlike synaptic vesicles (SVs), which are locally refilled and recycled at synaptic terminals in neurons, DCVs are filled with cargo at the Golgi in the cell soma and are then trafficked long distance along cytoskeletal elements with the help of motor proteins (Burgoyne and Morgan, 2003; Kim et al., 2006; Wong et al., 2012). So far, several studies have been done in PC-12 cells, other neuroendocrine cells, C.elegans, Drosophila, and mammalian neurons, but we still lack of complete understanding of how cargo-filled DCVs are transported, and captured at release sites. This motivated us to explore these properties in mammalian hippocampal neurons in the current study.

In PC-12 cells, Synaptotagmin 4 (Syt4), an integral DCV protein, is phosphorylated at S135 by JNK. This promotes a change in localization of Syt4 from immature DCVs at the Golgi to mature secretory DCVs away from the Golgi, and increases $\mathrm{Ca}^{2+}$ evoked release following NGF stimulation (Mori et al., 2008). It was also reported that in Drosophila neurons there is circuitous trafficking of DCVs in axons and active synapses capture these vesicles from the transiting pool when needed in a reversible and repeatable manner (Shakiryanova et al., 2006; Wong et al., 2012). In mammalian neurons, Syt4 is integral to brain-derived neurotrophic factor (BDNF)-harboring DCVs (Dean et al., 2009), which are recruited to synapses following increases in neuronal activity (Dean et al., 2012). In addition, JNK has also been reported to be involved in vesicle trafficking and unloading of kinesin transported cargoes from tubulin tracks in neurons (Stagi et al., 2006; Gibbs et al., 2015).

We hypothesized, based on all these evidences that the circuitous trafficking of DCVs observed in Drosophila might be the general phenomenon that also occurs in mammalian neurons. We further hypothesized that phosphorylation of Syt4 at S135 in neurons may be important for the trafficking, and capture of these DCVs at release sites in neuronal processes. To capture DCVs from transiting pool, highly active synapses might promote phosphorylation of JNK that could then phosphorylate transiting Syt4 vesicles at S135, causing them to detach from microtubules. Apart from capture, we also sought to 
investigate if phosphorylation of Syt4 at S135 affects fusion of DCVs at synaptic or nonsynaptic sites in hippocampal neurons.

This regulated transport, capture and release of DCVs in neurons would potentially allow fast recruitment of Syt4 containing DCVs to active synapses, bypassing the delays associated with signalling between synapses and the soma. This would in turn ensure robust maintenance and modulation of neurotransmission in hippocampal neurons via dense core vesicles. 


\section{Materials and Methods}

\subsection{Materials}

\subsubsection{Antibodies used}

All primary and secondary antibodies used in this dissertation are specified in Table 2 and Table 3 below:

Table 2 List of primary antibodies used

\begin{tabular}{|c|c|c|c|c|}
\hline Primary Antibodies & Host & Catalogue & Application & Company \\
\hline $\begin{array}{l}\text { Chromogranin A } \\
\text { (CgA) }\end{array}$ & Sheep & $2095-0220$ & ICC (1:2000) & AbD Serotec \\
\hline JNK/SAPK1 & Mouse & 610627 & $\begin{array}{l}\text { ICC (1:400) } \\
\text { WB (1:1000) }\end{array}$ & $\begin{array}{l}\text { BD } \\
\text { Transduction } \\
\text { Laboratories }\end{array}$ \\
\hline Kif1a & Mouse & 612094 & WB (1:500) & $\begin{array}{l}\text { BD } \\
\text { Transduction } \\
\text { Laboratories }\end{array}$ \\
\hline Map2 & Chick & 5543 & $\operatorname{ICC}(1: 3000)$ & Millipore \\
\hline Phospho- SAPK/JNK & Mouse & 9255 & $\begin{array}{l}\text { ICC (1:400) } \\
\text { WB (1:1000) }\end{array}$ & $\begin{array}{l}\text { Cell Signaling } \\
\text { Technology }\end{array}$ \\
\hline Phospho-c-Jun & Rabbit & 9261 & WB (1:1000) & $\begin{array}{l}\text { Cell Signaling } \\
\text { Technology }\end{array}$ \\
\hline PSD-95 & Mouse & 124011 & WB (1:1000) & $\begin{array}{l}\text { Synaptic } \\
\text { Systems }\end{array}$ \\
\hline Rab-GDI & Mouse & 130011 & WB (1:1000) & $\begin{array}{l}\text { Synaptic } \\
\text { Systems }\end{array}$ \\
\hline $\begin{array}{l}\text { Synaptobrevin } 2 \\
\text { (VAMP2) }\end{array}$ & Mouse & $\begin{array}{l}104211 \\
\text { (Clone 69.1) }\end{array}$ & WB (1:1000) & $\begin{array}{l}\text { Synaptic } \\
\text { Systems }\end{array}$ \\
\hline Synaptophysin & $\begin{array}{l}\text { Guinea } \\
\text { pig }\end{array}$ & 101004 & ICC (1:2000) & $\begin{array}{l}\text { Synaptic } \\
\text { Systems }\end{array}$ \\
\hline Synaptophysin & Mouse & $\begin{array}{ll}\text { 101011 } & \text { (Clone } \\
7.2) & \end{array}$ & WB (1:1000) & $\begin{array}{l}\text { Synaptic } \\
\text { Systems }\end{array}$ \\
\hline Synaptotagmin 1 & Mouse & 105101 & WB (1:1000) & $\begin{array}{l}\text { Synaptic } \\
\text { Systems }\end{array}$ \\
\hline
\end{tabular}




\begin{tabular}{|l|l|l|l|l|}
\hline Synaptotagmin 4 & Rabbit & 105043 & $\begin{array}{l}\text { ICC (1:1000) } \\
\text { WB (1:1000) }\end{array}$ & $\begin{array}{l}\text { Synaptic } \\
\text { Systems }\end{array}$ \\
\hline Tubulin & Mouse & 302211 & WB (1:1000) & $\begin{array}{l}\text { Synaptic } \\
\text { Systems }\end{array}$ \\
\hline
\end{tabular}

Table 3 List of secondary antibodies used

\begin{tabular}{|l|l|l|l|l|}
\hline $\begin{array}{l}\text { Secondary Antibodies } \\
\text { with conjugated dye }\end{array}$ & Host & $\begin{array}{l}\text { Catalogue } \\
\text { Number }\end{array}$ & Applications & Company \\
\hline $\begin{array}{l}\text { Anti- guinea pig Alexa } \\
647\end{array}$ & Goat & A-21450 & ICC (1:2000) & Invitrogen \\
\hline Anti- rabbit Alexa 546 & Goat & A-11010 & ICC (1:2000) & Invitrogen \\
\hline Anti-chick Alexa 405 & Goat & 175765 & ICC (1:2000) & Abcam \\
\hline Anti-mouse Alexa 488 & Donkey & A-21202 & ICC (1:2000) & Invitrogen \\
\hline $\begin{array}{l}\text { HRP-coupled } \\
\text { monoclonal mouse }\end{array}$ & Mouse & 1706516 & WB (1:2000) & Bio-Rad \\
\hline $\begin{array}{l}\text { HRP-coupled rabbit } \\
\text { polyclonal }\end{array}$ & Rabbit & 1706515 & WB (1:2000) & Bio-Rad \\
\hline $\begin{array}{l}\text { Anti- guinea pig Alexa } \\
647\end{array}$ & Goat & A-21450 & ICC (1:2000) & Invitrogen \\
\hline Anti- rabbit Alexa 546 & Goat & A-11010 & ICC (1:2000) & Invitrogen \\
\hline Anti-chick Alexa 405 & Goat & 175765 & ICC (1:2000) & Abcam \\
\hline
\end{tabular}

\subsubsection{Mammalian Expression constructs}

All plasmids used in this study are listed below in Table 4:

Table 4 List of all plasmids used in this study for over-expression

\begin{tabular}{|r|l|}
\hline Constructs Name & Source \\
\hline 1) Kif1a-GFP & $\begin{array}{l}\text { M.A. Silverman, Burnaby, BC, } \\
\text { Canada. }\end{array}$ \\
\hline 1) AAV-ESYN-mCherry-rSytIV ( Syt4 & $\begin{array}{l}\text { Jonathan T. Ting, Duke University, } \\
\text { control) } \\
\text { 2) }\end{array}$ \\
\begin{tabular}{l} 
(Syt4 phosphom, NC, United States \\
\hline
\end{tabular} & \\
\hline
\end{tabular}




\begin{tabular}{|c|c|c|}
\hline 3) & $\begin{array}{l}\text { AAV-ESYN-mCherry-rSytIV (S135E) } \\
\text { (Syt4 phosphomimetic) }\end{array}$ & \\
\hline $\begin{array}{l}\text { 1) } \\
\text { 2) } \\
\text { 3) } \\
\text { 4) } \\
\text { 5) }\end{array}$ & $\begin{array}{l}\text { Bassoon-GFP } \\
\text { CgA-GFP } \\
\text { LifeAct-RFP } \\
\text { PSD-95 GFP } \\
\text { Synaptophysin-GFP }\end{array}$ & $\begin{array}{l}\text { Thomas Dresbach, University of } \\
\text { Goettingen }\end{array}$ \\
\hline $\begin{array}{l}\text { 1) } \\
\text { 2) }\end{array}$ & $\begin{array}{l}\text { pAAV-SYN-mCherry-SytIV-P2A- } \\
\text { FlagJNK1a1(apf) [JNK1(APF)] } \\
\text { pAAV-SYN-mCherry-SytIV-P2A- } \\
\text { FlagMKK7B2Jnk1a1 [MKK7-JNK1] } \\
\text { pAAV-SYN-mCherry-SytIV(S135A)- } \\
\text { P2A-FlagMKK7B2Jnk1a1 } \\
\text { [S135A/MKK7-JNK1] }\end{array}$ & $\begin{array}{l}\text { Were cloned by using the insert from } \\
\text { pCDNA3 Flag MKK7B2Jnk1a1 } \\
\text { (Addgene \# 19726) or from pCDNA3 } \\
\text { Flag Jnk1a1 (apf) (Addgene \# 13846) } \\
\text { in Syt4 control or deficient construct } \\
\text { in addition to a P2A domain (Derijard } \\
\text { et al., 1994; Lei et al., 2002; Kim et } \\
\text { al., 2011) to ensure co-transfection. }\end{array}$ \\
\hline 1) & Syt4 (control)-pHluorin & $\begin{array}{l}\text { Edwin R. Chapman, University of } \\
\text { Wisconsin, Madison, Wisconsin }\end{array}$ \\
\hline 1) & $\begin{array}{l}\text { Syt4 (S135A)-pHluorin } \\
\text { Syt4 (S135E)-pHluorin }\end{array}$ & $\begin{array}{l}\text { Site directed mutagenesis was done } \\
\text { using the Syt4 control-pHluorin } \\
\text { construct to get these pHluorin } \\
\text { mutant constructs. }\end{array}$ \\
\hline
\end{tabular}

\subsubsection{Buffers and Solutions}

The various buffers and solutions used are listed in Table 5:

Table 5 List of buffers and solutions used

\begin{tabular}{|c|c|}
\hline Buffers and solutions & Ingredients \\
\hline Anode Buffer (10X) & $2 \mathrm{M}$ Tris in $\mathrm{dH}_{2} \mathrm{O}$ adjusted to $\mathrm{pH} 8.9$ with $\mathrm{HCl}$ \\
\hline Base Solution & $\begin{array}{l}140 \mathrm{mM} \mathrm{NaCl}, 5 \mathrm{mM} \mathrm{KCl}, 2 \mathrm{mM} \mathrm{CaCl}, 2 \mathrm{mM} \mathrm{MgCl}_{2}, 5.5 \mathrm{mM} \\
\text { glucose, } 20 \mathrm{mM} \text { Hepes, } \mathrm{pH}=7.3\end{array}$ \\
\hline Blot Buffer & 200 mM glycine, 25 mM Tris, 0.04\% SDS, 20\% methanol \\
\hline Buffer D & $\begin{array}{l}2 \% \text { donkey serum, } 0.1 \% \text { Triton } \mathrm{X}-100 \text {, and } 0.05 \% \text { sodium } \\
\text { azide in } 2 \times \text { PBS }\end{array}$ \\
\hline Cathode Buffer & $1 \mathrm{M}$ Tris, $1 \mathrm{M}$ Tricin, $1 \%$ SDS in $\mathrm{dH}_{2} \mathrm{O}(\mathrm{pH}=8.25)$. \\
\hline
\end{tabular}




\begin{tabular}{|c|c|}
\hline Dissection Media & $\begin{array}{l}\text { Hank's Balanced Salt Solution, HBSS (Gibco) buffered with } 10 \\
\text { mM HEPES (Gibco) }\end{array}$ \\
\hline Gel Buffer & $3 \mathrm{M}$ Tris- $\mathrm{HCl}(\mathrm{pH} 8.45), 0.3 \%$ (w/v) SDS \\
\hline HEK cells media & $\begin{array}{l}\text { DMEM (4.5 g/L glucose; [-] glutamine; [-] pyruvate, GIBCO) } \\
\text { supplemented with } 10 \% \text { FBS (Biochrom) and } 1 \% \\
\text { pencillin/streptomycin (Invitrogen) }\end{array}$ \\
\hline High $\mathrm{KCL}$ solution & $\begin{array}{l}100 \mathrm{mM} \mathrm{NaCl}, 90 \mathrm{mM} \mathrm{KCl}, 2 \mathrm{mM} \mathrm{CaCl} \text {, } 2 \mathrm{mM} \mathrm{MgCl}_{2}, 5.5 \mathrm{mM} \\
\text { glucose, } 20 \mathrm{mM} \text { Hepes, } \mathrm{pH}=7.3\end{array}$ \\
\hline $\begin{array}{l}\text { Homogenization } \\
\text { Buffer }\end{array}$ & 320 mM sucrose, 4 mM HEPES; pH 7.4, adjusted with $\mathrm{NaOH}$ \\
\hline Lysis buffer for tails & $\begin{array}{l}100 \mathrm{mM} \text { Tris- } \mathrm{HCl} \text { (pH 8.5), } 5 \text { mM EDTA (pH 8.0), 0.2\% SDS, } \\
200 \mathrm{mM} \mathrm{NaCl}\end{array}$ \\
\hline $\begin{array}{l}\text { Neurobasal }{ }^{\circledR} \text { Plus media } \\
\text { (NB+ media) }\end{array}$ & $\begin{array}{l}\text { Neurobasal }^{\circledast} \quad(\mathrm{NB}), 1 \times \text { B-27 supplement, 1x Glutamax, } \\
\text { penicillin/streptomycin: } 100 \mathrm{U}\end{array}$ \\
\hline $\mathrm{NH}_{4} \mathrm{Cl}$ Solution & $\begin{array}{l}90 \mathrm{mM} \mathrm{NaCl} 50 \mathrm{mM} \mathrm{NH}_{4} \mathrm{Cl}, 5 \mathrm{mM} \mathrm{KCl}, 2 \mathrm{mM} \mathrm{CaCl}, 2 \mathrm{mM} \\
\mathrm{MgCl}_{2}, 5.5 \mathrm{mM} \text { glucose, } 20 \mathrm{mM} \text { Hepes, } \mathrm{pH}=7.3\end{array}$ \\
\hline PBS & $20 \mathrm{mM} \mathrm{Na}_{2} \mathrm{HPO}_{4}, 150 \mathrm{mM} \mathrm{NaCl}, \mathrm{pH} 7.4$ \\
\hline SDS- Sample Buffer & $\begin{array}{l}50 \mathrm{mM} \text { Tris, } 4 \% \text { SDS, } 0.01 \% \text { Serva Blue G, } 12 \% \text { Glycerol to } \mathrm{pH} \\
6.8 \text { with HCl, } 2 \% \text { B-mercaptoethanol }\end{array}$ \\
\hline $\begin{array}{l}\text { Transfection buffer } \\
\text { (used in calcium } \\
\text { phosphate } \\
\text { transfection) }\end{array}$ & $\begin{array}{l}274 \mathrm{mM} \mathrm{NaCl}, 10 \mathrm{mM} \mathrm{KCl}, 1.4 \mathrm{mM} \mathrm{Na}_{2} \mathrm{HPO}_{4}, 15 \mathrm{mM} \text { glucose, } \\
42 \mathrm{mM} \text { Hepes, pH } 7.06\end{array}$ \\
\hline
\end{tabular}

\subsubsection{Chemicals used}

All chemicals used in various experiments are listed below in Table 6:

Table 6 Major chemicals/drugs used in this study

\begin{tabular}{|l|l|l|l|}
\hline Chemical Name & Role/Application & $\begin{array}{l}\text { Catalogue } \\
\text { Number }\end{array}$ & Company \\
\hline Anisomycin & $\begin{array}{l}\text { Acts as a JNK activator (and } \\
\text { inhibits protein synthesis) (Frey } \\
\text { et al., 1988; Moriguchi et al., } \\
\text { 1997; Curtin and Cotter, 2002) }\end{array}$ & Sigma-Aldrich \\
\hline
\end{tabular}




\begin{tabular}{|c|c|c|c|}
\hline Bicuculline & $\begin{array}{l}\text { Increases neuronal activity, is an } \\
\text { antagonist of } \mathrm{GABA}_{\mathrm{A}} \text { receptors } \\
\text { (Heyer et al., 1982; Dean et al., } \\
\text { 2012) }\end{array}$ & 120108 & Abcam \\
\hline Latrunculin B & $\begin{array}{l}\text { Sequesters G-actin and prevents } \\
\text { F-actin assembly (Wakatsuki et } \\
\text { al., 2001; Colin et al., 2008) }\end{array}$ & 3973 & Tocris \\
\hline Nocodazole & $\begin{array}{l}\text { Interferes with the } \\
\text { polymerization of microtubules } \\
\text { (Colin et al., 2008) (Eilers et al., } \\
\text { 1989) }\end{array}$ & 1228 & Tocris \\
\hline SP600125 & $\begin{array}{l}\text { Inhibitor of JNK (Bennett et al., } \\
\text { 2001; Mori et al., 2008) }\end{array}$ & S5567 & Sigma \\
\hline
\end{tabular}

\subsubsection{Mouse lines}

Syt4 wild type or knockout mice (provided by Harvey Herschman, UCLA) were used for immunocytochemistry experiments or brain lysate preparation for Western blotting.

\subsubsection{Genotyping}

Mouse tail biopsy was used to isolate DNA for genotyping. Tail samples obtained from the animal facility were digested with $3.5 \mu$ of $10 \mathrm{mg} / \mathrm{ml}$ proteinase $\mathrm{K}$ in $500 \mu \mathrm{l}$ lysis buffer (Table 5) while shaking for 6 hours or overnight at $55^{\circ} \mathrm{C}$. Samples were then centrifuged in a tabletop centrifuge at maximum speed for $10 \mathrm{~min}$ and supernatants were transferred into a fresh tube. These were then precipitated with $500 \mu \mathrm{l}$ isopropanol and mixed well. Samples were then centrifuged again at maximum speed for $10 \mathrm{~min}$, after which supernatants were removed and $200 \mu \mathrm{l} 70 \%$ ethanol were used to wash each pellet by centrifuging for another $10 \mathrm{~min}$ at maximum speed. Next, the ethanol was removed and the pellets dried for subsequent re-suspension in $100 \mu \mathrm{ldd} \mathrm{H}_{2} \mathrm{O}$.

Genotyping using the DNA isolated from the mouse tails was carried out using specific primers:

Syt4 Wt forward primer: 5'-CACTTCCCTCACGTCAGAGGAG-3'

Syt4 KO forward primer: 5'-AACCACACTGCTCGACATTGGG-3'

Syt4 Reverse primer (for both WT and KO): 5'-GCAAGGAGAGCTCTTGGATGTG-3' 
Wild-type (WT) band is $300 \mathrm{bp}$

Knockout (KO) band is $250 \mathrm{bp}$

For each sample, $2 \mu \mathrm{I}$ DNA were transferred to fresh PCR tubes, and mixed with $48 \mu \mathrm{l}$ of the following PCR mix:

\begin{tabular}{ll} 
Reagent & Volume \\
\hline 10X Buffer & $5 \mu \mathrm{l}$ \\
$25 \mathrm{mM}$ dNTPs & $0.4 \mu \mathrm{l}$ \\
$10 \mu \mathrm{M}$ forward primer & $1 \mu \mathrm{l}$ \\
$10 \mu \mathrm{M}$ reverse primer & $1 \mu \mathrm{l}$ \\
Taq DNA polymerase & $1.25 \mu \mathrm{l}$ \\
$\mathrm{d} \mathrm{H}_{2} \mathrm{O}$ & $39.25 \mu \mathrm{l}$ \\
Total & $48 \mu \mathrm{l} /$ reaction $(+2 \mu \mathrm{l}$ of DNA $)$ \\
\hline
\end{tabular}

Samples were then placed in thermocyclers for PCR to amplify specific DNA segments (Saiki et al., 1988; Mullis et al., 1992). The genotyping PCR thermocycler program was as follows:

\section{PCR program:}

$93{ }^{\circ} \mathrm{C}$ for $10 \mathrm{~min}$

Cycle $(40 \mathrm{X})$ :

$93^{\circ} \mathrm{C}$ for $30 \mathrm{sec}$

$60{ }^{\circ} \mathrm{C}$ for $45 \mathrm{sec}$

$65^{\circ} \mathrm{C}$ for $90 \mathrm{sec}$

$65^{\circ} \mathrm{C}$ for $10 \mathrm{~min}$

$4{ }^{\circ} \mathrm{C}$ hold

Then, to separate the PCR products, $2 \%(\mathrm{w} / \mathrm{v})$ agarose gels in 1 XTAE buffer (including SYBR ${ }^{\circledR}$ Safe DNA Gel Stain, ThermoFisher Scientific) were loaded with PCR samples and $100 \mathrm{bp}$ DNA ladder (ThermoFisher Scientific, Germany) in a separate lane. DNA bands were separated by horizontal gel electrophoresis at $75 \mathrm{~V}$ for $1 \mathrm{~h}$. To document genotyping results, pictures were obtained using a UV illuminator with the INTAS imaging system. 


\subsection{Methods}

\subsubsection{Cell Culture}

All research involving animals was approved by the Institutional Animal Care and Ethics Committees of Goettingen University (T10.31), and was done in accordance with German animal welfare laws. We tried our best to minimize the number of animals used and their suffering. All animals used for experiments in this dissertation were bred and kept in the mouse facility of the European Neuroscience Institute or the animal facility of the University Medical Center in Göttingen, Germany.

\subsubsection{Dissociated rat hippocampal neuron culture preparation}

$12 \mathrm{~mm}$ glass coverslips (Carolina Biologicals) stored in $70 \%$ ethanol were transferred one by one into 24-well plates and washed thrice with autoclaved distilled water. These coverslips were then coated with either $0.04 \%$ polyethyleneimine ( $\mathrm{PEI}$, Sigma) or $0.5 \mathrm{mg} / \mathrm{ml}$ poly-D-lysine (PDL, Sigma). PEl coating was done overnight at $37{ }^{\circ} \mathrm{C}$ whereas PDL coating was done for 4 hours at room temperature. These coated coverslips were then washed and stored in distilled water.

For dissociated hippocampal neuronal cultures, the protocol published in (Banker and Cowan, 1977) was used with minor modifications. E18-19 wistar rats were used for rat hippocampal culture preparation. After euthanizing timed pregnant rats with $\mathrm{CO}_{2}$, embryos were removed and brains of embryos were transferred into $4{ }^{\circ} \mathrm{C}$ dissection media (Table 5). Once meninges were removed carefully from isolated brains, the hippocampi were cut out using fine forceps from both the hemispheres of each brain. Then isolated hippocampi were put into a $15 \mathrm{~mL}$ falcon tube containing $4{ }^{\circ} \mathrm{C}$ dissection media. Following collection of all hippocampi, the dissection media in the falcon tube was aspirated. $2 \mathrm{~mL}$ pre-warmed $0.05 \%$ trypsin (Gibco) at 37 o C was added to the hippocampi and the solution was incubated for $20 \mathrm{~min}$ in a $37^{\circ} \mathrm{C}$ water bath. After incubation, trypsin solution was aspirated carefully and tissue was washed 3 times with $5 \mathrm{~mL} 4{ }^{\circ} \mathrm{C}$ dissection media. Following the third wash, $1 \mathrm{~mL} \mathrm{NB}+$ (Table 5) was added and cells were triturated to obtain a single cell suspension. Afterwards, the cell suspension was filtered through a $100 \mu \mathrm{m}$ cell strainer (BD Falcon) prewet with $4.5 \mathrm{~mL}$ culture media. After filtration, the strainer was washed with another 4.5 $\mathrm{mL}$ culture media so that the final cell suspension was $10 \mathrm{~mL}$. Finally, cell number was determined using a hemocytometer with trypan blue staining. For rat hippocampal 
neuronal cultures, the cell suspension was adjusted to 80,000 cells $/ 500 \mu \mathrm{L}$. Thereafter, 500 $\mu \mathrm{L}$ of cell suspension was added to each well on coated coverslips. Cultures were maintained in a Hera Cell $240 \mathrm{i}$ cell culture incubator (Thermo Scientific) at $37^{\circ} \mathrm{C}$ and $5 \%$ $\mathrm{CO}_{2}$.

\subsubsection{Dissociated mouse hippocampal neuron culture preparation}

PO Syt4 WT/KO mice were used for mouse hippocampal neuronal preparation. Brains were removed directly following decapitation and put into cold dissection media. After this step, the protocol was similar to rat hippocampal neuron preparation with minor modifications. Briefly, isolated hippocampi were kept in $0.25 \%$ of trypsin (Gibco) for tissue digestion in the water bath for 30 minutes. All other steps including trituration, counting and plating were similar to rat hippocampal culture preparation. Mouse hippocampal neurons were plated with comparatively higher density of 100,000 cells /500ul on PDL or PEI coated coverslips. Cultures were maintained in a Hera Cell 240i cell culture incubator (Thermo Scientific) at $37^{\circ} \mathrm{C}$ and $5 \% \mathrm{CO}_{2}$.

\subsubsection{HEK 293T cell culture}

HEK 293 T cells were grown in HEK cell media (Table 5) in $10 \mathrm{~cm}$ culture dishes. Cells were split when the plate was approximately $90 \%$ confluent. These confluent cells were then washed with $5 \mathrm{~mL}$ pre-warmed PBS after removing the media. $1 \mathrm{~mL}$ of $0.25 \%$ trypsin (Gibco) was added for $2 \mathrm{~min}$ to reduce the adherence of cells from the dish. The adhering cells were further removed by adding $9 \mathrm{~mL}$ of HEK cell medium and the cells were pipetted up and down several times to obtain single-cell suspension. The cell suspension was centrifuged at $800 \mathrm{rpm}$ for $5 \mathrm{~min}$ and then the pellet was resuspended in $5 \mathrm{~mL}$ of HEK cell media. The cells were then plated on $10 \mathrm{~cm}$ culture dishes to obtain desired confluency and kept in the incubator.

\subsubsection{Transfection Protocols}

Two different transfection protocols were performed to overexpress desired plasmids. For hippocampal neurons, Lipofectamine 2000 transfection was used and for HEK cells, calcium phosphate transfection was used. Plasmid DNA (Table 4) used for transfection was first amplified using the following protocol. 


\subsubsection{Plasmid DNA amplification}

E. coli strain XL-1 Blue electrocompetent cells (Stratagene, cat. no.: 200249) were used to amplify DNA. $2 \mu \mathrm{l}$ of plasmid DNA was added to an electroporation cuvette (Biozym Scientific $\mathrm{GmbH}$, cat. \# 748010) with $50 \mu$ l E.coli XL1-blue cells. This mixture was then treated with an $1800 \mathrm{~V}$ impulse in an electroporator 2510 (Eppendorf). $1 \mathrm{~mL}$ LB (Roth, cat. no.: X968.1; prepared according to the manufacturer's recommendations) was then added to the cuvette, mixed with the cell suspension, and then transferred into a new tube. This tube was then incubated on an eppendorf thermomixer (for $1.5 \mathrm{ml}$ tubes) at $37^{\circ} \mathrm{C}$ for $1 \mathrm{~h}$ with shaking at $800 \mathrm{rpm}$. Using an ethanol-sprayed, bent glass pipette, $20 \mu \mathrm{l}$ of the bacterial mix was spread over a $10 \mathrm{~cm}$ LB-agar plate (that contained $0.5 \%$ yeast extract, $1 \%$ tryptone (pH 7), $1 \% \mathrm{NaCl}$, and $1.5 \%$ agar in LB medium), together with $100 \mu \mathrm{g} / \mathrm{mL}$ ampicillin (Roth, cat. no.: K029.2) or $50 \mu \mathrm{g} / \mathrm{mL}$ kanamycin (Roth, cat. no.: T832.3)). Plates were then incubated at $37^{\circ} \mathrm{C}$ overnight. Afterwards, $20 \mu \mathrm{l}$ of cell suspension was smeared on a LB agar plate with the required antibiotic and grown overnight at $37^{\circ} \mathrm{C}$ in the incubator. The next day, single isolated bacterial colonies were selected and each was added to an Erlenmeyer flask containing $300 \mathrm{~mL}$ pre-warmed LB medium with $50 \mu \mathrm{g} / \mathrm{mL}$ of the respective antibiotic. Flasks were incubated with shaking at $300 \mathrm{rpm}$ and $37^{\circ} \mathrm{C}$ for $16-24 \mathrm{~h}$. These cultures were then used for plasmid purification.

For plasmid purification, the NucleoBond ${ }^{\circledR}$ Xtra Maxi-Kit from Macherey-Nagel with the suggested protocol was used. The centrifugation steps were done at $4000 \mathrm{~g}$ and $4{ }^{\circ} \mathrm{C}$ for double the time suggested in the protocol. DNA pellets were reconstituted with an appropriate amount of $\mathrm{ddH}_{2} \mathrm{O}$. DNA concentration was measured using a NanoPhotometer Implen (Montreal Biotech Inc.). The constructs were then stored at $-20^{\circ} \mathrm{C}$ until use for transfection.

\subsubsection{Lipofectamine 2000 Transfection}

Neurons were transfected at days in vitro 10 (DIV 10) using Lipofectamine 2000 (Invitrogen). 1uL of Lipofectamine 2000 in $50 \mu \mathrm{L}$ of Neurobasal medium and $0.75 \mu \mathrm{g}$ of DNA in 50 uL of Neurobasal (Gibco) medium were incubated separately for $5 \mathrm{~min}$. Then the DNA solution was mixed with the Lipofectamine solution and incubated for $20 \mathrm{~min}$ at room temperature. Meanwhile, conditioned culture medium (in which cells were growing for the past 10 days) was removed, saved and replaced with $400 \mu \mathrm{L}$ of fresh prewarmed 
Neurobasal medium. The saved conditioned media was stored at $37{ }^{\circ} \mathrm{C}$ and $5 \% \mathrm{CO}_{2}$. After 20 min incubation time, $100 \mu \mathrm{L}$ of Lipofectamine-DNA mixture was added to the well and incubated for four hours at $37{ }^{\circ} \mathrm{C}$ and $5 \% \mathrm{CO}_{2}$. The transfection medium was then removed, cells were washed once with $500 \mu \mathrm{L}$ of Neurobasal pre-warmed to $37{ }^{\circ} \mathrm{C}$ and then exchanged with $500 \mu \mathrm{L}$ of $37{ }^{\circ} \mathrm{C}$ stored conditioned media. Media was immediately replaced after removing the transfection media to avoid exposing neurons to air. After transfection, cells were placed back in the incubator at $37{ }^{\circ} \mathrm{C}$ and $5 \% \mathrm{CO}_{2}$. These transfected cells were then imaged between DIV 13-16.

\subsubsection{Calcium phosphate transfection}

HEK 293 T cells were transfected using calcium phosphate when they were $40-50 \%$ confluent. To prepare the DNA-Ca ${ }^{2+}$ precipitate for one $10 \mathrm{~cm}$ culture dish, $1.3 \mathrm{~mL}$ of transfection buffer (Table 5) was added dropwise to another $1.3 \mathrm{~mL}$ of $\mathrm{ddH}_{2} \mathrm{O}$ containing $20 \mu \mathrm{g}$ of DNA and $166 \mu \mathrm{L}$ of $2 \mathrm{M} \mathrm{CaCl}_{2}$, under gentle vortex. This complete transfection mixture was then added dropwise to the HEK cells culture plate. The culture was then incubated with this transfection mixture overnight at $37{ }^{\circ} \mathrm{C}$ and $5 \% \mathrm{CO}_{2}$. The next day, media was removed and culture was washed twice with prewarmed PBS. After washing, fresh HEK cells media (Table 5) was added to the cells. These transfected HEK cell cultures were then grown until they were $90-95 \%$ confluent and were then used for immunoprecipitation experiments.

\subsubsection{Imaging protocols}

Both fixed sample and live cell imaging were done in this study. Details of the imaging setup and description of imaging protocols are as follows:

\subsubsection{Immunocytochemistry (ICC) and fixed sample imaging}

For immunocytochemistry, cultured neurons were fixed at DIV $14-16$ for $30 \mathrm{~min}$ in $4 \%$ paraformaldehyde in 0.1 M phosphate buffer (Bhatnagar, 1996). These cells were then washed in $1 \mathrm{X}$ PBS for $3 \times 3 \mathrm{~min}$. Fixed samples were then stored in $1 \mathrm{X}$ PBS at $4{ }^{\circ} \mathrm{C}$ or immediately used for immunostaining. To prevent non-specific binding and permeablize the cells, samples were incubated with buffer D (Table 5) for $30 \mathrm{~min}$. After blocking and permeabilization, primary antibodies (diluted in buffer D) were added onto the samples overnight at $4{ }^{\circ} \mathrm{C}$. The following day, samples were washed in 1 X PBS for $3 \times 3 \mathrm{~min}$, after 
which secondary antibodies (diluted in buffer D) were added and incubated at room temperature in the dark for $2 \mathrm{~h}$. After secondary antibody incubation, samples were washed in 1X PBS for $3 \times 3 \mathrm{~min}$. Next, the coverslips were mounted in Fluoromount mounting reagent (Diagnostic BioSystems, cat. \# K048) on glass slides. After mounting, the

edges of coverslips were sealed with transparent nail polish. Samples were stored at $4{ }^{\circ} \mathrm{C}$ in the dark and were then imaged. Fixed sample imaging was done using a 40X/ 1.3 NA oil DIC objective (Zeiss, Plan-Apochromat) on a Zeiss LSM 710 confocal microscope. Images were analyzed using ImageJ software (National Institute of Health, USA, Plug-in Coloc2) or MetaMorph.

\subsubsection{Live cell imaging}

For live cell imaging, transfected neurons on coverslips at DIV 13-15 were transferred to a live imaging chamber (Warner Instruments) containing $150 \mu \mathrm{L}$ of Base solution (Table 5). Images of GFP-tagged constructs were acquired with 450-490ex/505-555em and of mCherry-tagged constructs with 545-570ex/575-680em on a Zeiss AxioObserver inverted microscope with a Photometrics Evolve EMCCD camera, and Lambda DG-4 high-speed wavelength switcher interfaced with Metamorph software.

Trafficking Experiments: Healthy neurons or regions were selected during live cell imaging based on cell morphology and in which vesicles could be seen moving within the field of view before acquiring videos. Images were acquired at $100 \mathrm{~ms}-500 \mathrm{~ms}$ exposure time at 1 sec intervals for $5 \mathrm{~min}$ and in some experiments for $20 \mathrm{~min}$. For experiments testing the impact of different pharmacological treatments (Table 6), cells were treated as follows: Nocodazole $(10 \mu \mathrm{M})$ and Latrunculin $(10 \mu \mathrm{M})$ for $30 \mathrm{~min}$, SP600125 $(10 \mu \mathrm{M})$ and Anisomycin $(50 \mu \mathrm{g} / \mathrm{mL})$ for $2-4 \mathrm{~h}$ each before bringing for imaging. Videos were then analysed using Metamorph and Imaris 7.6.4 (Bitplane).

For activity dependent pause experiments, high $\mathrm{KCL}$ solution (Table 5) was applied by pipette for 3 minutes on the scope itself during the time-lapse recording followed by perfusion with base Tyrode's solution to wash out high $\mathrm{KCL}$ solution. Kymographs were then made using kymograph tool in Metamorph software.

Fusion Experiments: Transfected cells (identified by faint GFP fluorescence in nondepolarizing conditions) in which axons and dendrites were clearly discernible by 
morphology were selected. Images of pHluorins were acquired at $1 \mathrm{sec}$ intervals for $3 \mathrm{~min}$. A baseline of twenty images was collected before addition of high KCL buffer (Table 5) to depolarize neurons. Axonal regions were selected in Metamorph and the fluorescence intensity was plotted versus time. Puncta that did not exhibit any lateral movement during image acquisition were chosen for analysis. For measurement of the surface versus internal amount of pHluorin signal, neurons were first depolarized with high $\mathrm{KCl}$ solution to confirm that recycling events could be detected within the field of view. Subsequent treatment with $\mathrm{NH}_{4} \mathrm{Cl}$ solution (Table 5) was then performed to dequench internal pHluorins and determine total pHluorin signal at regions at which vesicles fused.

\subsubsection{Biochemistry Experiments}

These experiments were done with Dr. Saheeb Ahmed (a postdoc in our lab).

\section{Sample preparation for immunoblotting}

For immunoblotting from cell cultures, dissociated hippocampal cells at DIV 13-15 or HEK 293 cells were cultured as described in sections 2.2 .1 on polystyrene based $10 \mathrm{~cm}$ tissue culture dishes (CellStar). For some experiments, pharmacological treatments were performed as described in the Results section before sample collection. To collect samples, culture medium from the plates was removed and cells were washed with 1X PBS (SigmaAldrich). Cells were then harvested in $500 \mu \mathrm{L} 1 \mathrm{X}$ PBS using a cell scraper (CytoOne, Cat. No.: \#CC76000220) and passed through a 27-gauge needle (HSW Fine-ject, cat.no.: \#4710004020). Lysate was then centrifuged at 4000 rpm in an Eppendorf centrifuge 5424 for $10 \mathrm{~min}$ at $4{ }^{\circ} \mathrm{C}$ to pellet nuclei and cellular debris.

In some experiments, whole brain homogenates were also used for immunoblotting. For these experiments, brains were isolated from 2-4 months old wild type or Syt4 knockout mice and transferred to a petri dish with $4{ }^{\circ} \mathrm{C}$ freshly prepared homogenization buffer on ice. Brains were chopped into smaller pieces and collected in $1.5 \mathrm{~mL}$ Eppendorf tubes containing $500 \mu \mathrm{L}$ of homogenization buffer. Homogenization of brain tissue was done in 9 $\mathrm{mL}$ of $4{ }^{\circ} \mathrm{C}$ homogenization buffer using a glass-teflon homogenizer (10 strokes at $900 \mathrm{rpm}$ ) in the cold room. Tubes with homogenized brain lysates were centrifuged at 1,000 X g for $10 \mathrm{~min}$ at $4{ }^{\circ} \mathrm{C}$. Supernatant was aliquoted and snap-frozen in liquid nitrogen for later use at $-80^{\circ} \mathrm{C}$. 
Protein concentration was determined using a BCA protein assay kit (Novagen, cat. no. \#712853) according to the manufacturer's recommendations.

\subsubsection{SDS-PAGE}

Protein fractionation was done on $10 \%$ denaturing Tris-SDS polyacrylamide gels using an electrophoresis system. Before running SDS-PAGE, gels were cast by mounting glass plates, spacers $(0.8 \mathrm{~mm})$ and combs (10 and 15 wells) onto a Bio-Rad holder. The components of the stacking gel and resolving gels were mixed as per the table shown below (Schägger and von Jagow, 1987).

\begin{tabular}{lll} 
Components & Stacking gel & Resolving gel \\
\hline 30\% Acrylamide & $200 \mu \mathrm{l}$ & $1.66 \mathrm{ml}$ \\
Gel buffer & $375 \mu \mathrm{l}$ & $1.68 \mathrm{ml}$ \\
Distilled water & $925 \mu \mathrm{l}$ & $570 \mu \mathrm{l}$ \\
$50 \%$ glycerol & - & $1.06 \mathrm{ml}$ \\
TEMED & $2 \mu \mathrm{l}$ & $3 \mu \mathrm{l}$ \\
$10 \%$ APS & $10 \mu \mathrm{l}$ & $25 \mu \mathrm{l}$ \\
\hline
\end{tabular}

First, $3.4 \mathrm{ml}$ of resolving gel (10\%) was poured and filled with $1 \mathrm{ml}$ of the stacking gel on top of it, to which a comb was added for casting. The outer gel running chamber was filled with anode buffer and cathode buffer was added to the inner cassette, into which gels were placed (Table 2.1.4). The samples were boiled in 5 X SDS-sample buffer (Table 5) for $5 \mathrm{~min}$ at $95{ }^{\circ} \mathrm{C}$ before being equally loaded into the lanes of the gel. For each gel, $5 \mu \mathrm{L}$ of prestained protein ladder (Fermentas, cat. no. \#SM0671) was loaded into one of the lanes. Electrophoresis of protein samples was then run at $60 \mathrm{~V}$ for $15 \mathrm{~min}$, and then $120 \mathrm{~V}$ for an hour.

\subsubsection{Western blotting}

After gel electrophoresis, separated proteins on gels were transferred onto nitrocellulose membranes. Before transfer, gels were equilibrated in blotting buffer for $10 \mathrm{~min}$ along with 
fibre pads, nitrocellulose membranes and filter papers. The blotting "sandwich" in the blot transfer apparatus was stacked as follows (cathode to anode end): one fibre pad, one filter paper, the gel, one nitrocellulose membrane, one filter paper, one fibre pad. The transfer was run at $100 \mathrm{~mA}$ for $1 \mathrm{~h}$ using a Bio-rad PowerPac 1000 blotting system.

After transfer, membranes were blocked with $5 \%$ milk solution in PBST $(0.05 \%$ Tween-20 in 1 XPBS) for $1 \mathrm{~h}$. The membranes were then incubated with primary antibodies (diluted appropriately in 5\% milk PBST) overnight. Following primary antibody incubation, blots were washed three times, 10 min each, with milk solution and then incubated with secondary antibodies in 5\% milk in PBST for $2 \mathrm{hr}$ at RT. Subsequently, each membrane was washed in $1 X$ PBS for $5 \times 5$ min, followed by applying ECL solution: ECL A (1.28\% (w/v) Tris$\mathrm{HCl}$ and $0.23 \%(w / v)$ luminol $\mathrm{Na}^{+}$salt (Sigma, cat. no.: A4685-1g) in $\mathrm{dH}_{2} \mathrm{O}(\mathrm{pH}$ 8.6), ECL B 0.01\% P-coumaric acid (Sigma, cat. no.: 9008-5g) in DMSO and ECL C solution $0.008 \% \mathrm{H}_{2} \mathrm{O}_{2}$ to the membrane for $1 \mathrm{~min}$. Antibody signal on membranes were detected using the Fujifilm LAS-3000 imaging system (R\&D Systems).

\subsubsection{Co-immunoprecipitation}

For co-immunoprecipitation analysis, transfected HEK 293 cells were harvested in IP-Lysis buffer (50 mM Tris-HCl pH 7.5; 150 mM NaCl; 2 mM EDTA; 0.5\%; NP40; Complete protease inhibitor (Roche)). Cell lysates were then incubated with $30 \mu \mathrm{L}$ of antibody coupled Protein A/G dynabeads (Invitrogen) or GFP-Trap beads (Chromotech) for $2 \mathrm{~h}$ at $4{ }^{\circ} \mathrm{C}$ on a rotator. Uncoupled beads were used for non-specific binding controls. Supernatant was saved as unbound fraction and bound proteins from beads were eluted by incubating them for 10 minutes at $95^{\circ} \mathrm{C}$ in $4 \times$ SDS sample buffer. The samples were then analyzed using SDS-PAGE and Western blotting.

\subsubsection{Immuno-isolation of synaptic vesicles}

Mouse monoclonal antibodies directed against Syt1 or Syb 2 were coupled to Protein A dynabeads (Invitrogen) in $1 \mathrm{mM}$ PBS-EDTA for $1 \mathrm{~h}$. at $4{ }^{\circ} \mathrm{C}$. Lysate was added to beads coupled with antibody and incubated for $2 \mathrm{~h}$. at $4{ }^{\circ} \mathrm{C}$ on a rotator. Magnetic beads were then separated from the non-bound fraction (supernatant) and washed three times with 1 mM PBS. Beads were then resuspended in sample buffer (bound fraction). Input, bound 
fraction and unbound fraction were separated by $12.5 \%$ SDS-PAGE and analyzed by Western blot using antibodies directed against Syt4, Syt1, Syp, Syb2, Rab-GDI and PSD-95.

\subsubsection{Subcellular fractionation of mouse brain}

Fractionation was performed as previously described (Ahmed et al., 2013). Briefly, mouse brain was homogenized in homogenization buffer ( $320 \mathrm{mM}$ sucrose, $4 \mathrm{mM}$ HEPES-KOH, pH 7.4) in a glass-teflon homogenizer (10 strokes at $900 \mathrm{rpm}$ ). The resulting homogenate $(\mathrm{H})$ was differentially centrifuged to obtain fractions.

\subsubsection{In-vitro kinase assay}

Four different peptides were made including S135 Syt4 and S63 c-Jun, known kinase motifs in Syt4 and c-Jun, to test Syt4 phosphorylation using an in vitro kinase assay according to the manufacturer's instructions.

Peptides were as follows:

Syt4 (WT): PETEKEAVSPESLKSST

Syt4 (def): PETEKEAVAPESLKSST

c-Jun (WT): AKNSDLLTSPDVGLLKL

c-Jun (def): AKNSDLLTAPDVGLLK

A JNK1 kinase assay with the ADP-Glo ${ }^{T M}$ Kinase assay kit was used (Promega). In all assays $0.2 \mu \mathrm{g} / \mu \mathrm{l}$ of each peptide and $5 \mu \mathrm{M}$ of ATP were used. First, standard curves were obtained by making a series of ADP-ATP dilutions based on the kit protocol, and the luminescence of each dilution was measured. Then phosphorylation of wild-type Syt4 and c-jun peptides by JNK1 were calculated based on standard curves and reported as \% activity (inclusive of auto-phosphorylation). In addition, phosphodeficient mutants were used as a control for specificity of the kinase at the desired site. The kinase activity was measured and reported relative to the value of the corresponding wild-type peptides. We used P38 peptides as positive control for JNK1 activity. This experiment was performed with our collaborator, Mahdokht Kohansal, a PhD student in Prof. Reinhard Jahn's lab. 


\subsubsection{Kinase prediction (in-silico approach)}

The GPS 3.0 (Zhou et al., 2004) was used as a tool to predict kinases that phosphorylate Syt4 at S135 and c-Jun (control peptide) at S63 of c-Jun. Peptides for wild-type Syt4: PETEKEAVSPESLKSST and wild-type c-Jun: AKNSDLLTSPDVGLLKL were submitted to the prediction software with a threshold set to medium (which corresponds to a false positive rate (FPR) equal to 6). All available serine/threonine kinases were activated as potential kinases. The software reported a score and a cut off value for each kinase. All kinases with Score/Cutoff $>2$ were assumed as significant predicted kinases for phosphorylation of the submitted sites. This experiment was done by our collaborator, Mahdokht Kohansal, a PhD student in Prof. Reinhard Jahn's lab.

\subsubsection{Quantification and data analysis}

\subsubsection{Co-localization analysis}

For co-localization analysis, we used ImageJ software (National Institute of Health, USA, Plug-in Coloc2). Pearson's coefficients were calculated to obtain cell wide correlation of fluorescence intensities (Farina et al., 2015). In activity-dependent capture experiments, the percentage of colocalization was determined as the percentage of the total thresholded area of one channel that overlapped with the thresholded area of the other channel using Metamorph (Dean et al., 2009).

\subsubsection{Live cell imaging analysis}

After acquisition, DCV mobility was tracked using the particle-tracking module of Imaris 7.6.4 (Bitplane). Positional data of vesicles was also obtained using this software.

For quantification of vesicle movement and pausing, positional data were imported into Matlab (Mathworks, Natick, MA, USA) and analyzed with custom-written programs. Matlab code was written by our collaborator Michael Siebrecht, MSc student in the lab. Vesicles that were present for less than $3 \mathrm{~s}$ within the field of view were not included in analysis. Track Speed Mean and Track Displacement was calculated for each condition. The mobile vesicle percentage was calculated as the percent of vesicles in each video with a track duration of at least $10 \mathrm{~s}$ and track displacement of at least $1 \mu \mathrm{m}$. For pause analysis, a pause was defined as a drop of vesicle velocity to $0 \pm 0.1 \mu \mathrm{m} / \mathrm{s}$ (Bury and Sabo, 2011). Average 
pause time was calculated only for those vesicles that could be tracked for the entire duration of the movie. Vesicles that were moving or paused for the entire duration of a time-lapse were excluded from average pause time analysis. Vesicles were used for $n$ number of all trafficking parameters except mobile percentage (for which video number was used), since measured parameters - determined by variance and standard deviation analysis - were the most variable for vesicles, compared to videos including many vesicles. Multiple axons from different transfected cells were imaged in each region selected for trafficking analysis, in which axons could not be traced back to specific cells. For this reason we did not use number of cells as the n number.

\subsubsection{Activity-dependent pause analysis}

For analysing activity-dependent pauses, immobile vesicles were not considered. A vesicle was considered paused during stimulation only if it was visible for the entire duration of $\mathrm{KCl}$ stimulation, and paused at or after stimulation for at least two thirds of the total time of stimulation (i.e. for $2 \mathrm{~min}$ ). Vesicles that satisfied these criteria were then counted to calculate percentage of moving vesicles that paused during stimulation.

Statistical significance for all quantification was determined by a Student's t test. For data having multiple comparisons, significance was evaluated using Student's t-test with Bonferroni correction. 



\section{Results}

\subsection{Syt4 is present on DCVs, distinct from SVs proteins}

To understand the trafficking of dense core vesicles, we first verified the localization of Syt4 in hippocampal neurons using a Syt4 antibody. Syt4 was previously shown to be present on DCVs in PC12 cells (Vician et al., 1995; Yoshihara and Montana, 2004) and on BDNFcontaining DCVs in hippocampal neurons (Dean et al., 2009). We confirmed the specificity of the Syt4 antibody using wild-type and Syt4 KO neurons. We found that Syt4 was prominent in the Golgi and was also in neurites. It showed a punctate distribution in wildtype neurons only, and was absent in Syt4 knockout by immunocytochemistry (ICC) (Fig 3.1 A). Syt4 has been reported to localize to BDNF-containing DCVs in hippocampal neurons, but Syt4 vesicles devoid of BDNF have also been observed (Dean et al., 2009). To test if Syt4 is a general marker of DCVs, we tested its co-localization with Chromogranin A $(\operatorname{CgA})$, a known cargo in the majority of DCVs, and compared it with a synaptic vesicle marker, synaptophysin (Syp) (Adams et al., 1993; McMahon et al., 1996; Machado et al., 2010). We found Syt4 highly co-localized with CgA in hippocampal neurons (Fig 3.1 B and C). $68.3 \pm$ $1.5 \%$ of CgA vesicles co-localized with Syt 4 and $82.4 \pm 1.1 \%$ of Syt 4 vesicles had $\operatorname{CgA}$, which was significantly higher than the colocalization of Syt4 and Syp (24.1 $\pm 2.6 \%$ of Syt 4 vesicles colocalized with Syp and $29.8 \pm 3.7 \%$ Syp vesicles were Syt4 positive) ( $n=6$ images for CgA and Syt4, 18 images for Syp and Syt4 staining). Because we planned to use mCherry-tagged Syt4 in trafficking experiments, we also tested if mCherry-Syt4 and CgA-GFP co-localized. We found a similar percentage of colocalization $(77.3 \pm 3.4 \%$ of CgA-GFP vesicles were Syt4-mCherry positive and $82.2 \pm 2.2 \%$ of Syt4-mCherry vesicles were co-localized with CgA-GFP) (n= 10 images for Syt4-mcherry and CgA-GFP co-transfection).

We further confirmed the specificity and localization of Syt4 by biochemistry experiments. We found a single band corresponding to the molecular weight ( $47 \mathrm{kDa}$ ) of Syt 4 in wildtype brain lysates, but not in Syt4 knockout lysates, by Western blotting (Fig 3.1 D). Also, to check localization of Syt4, we carried out subcellular fractionation from mouse brain homogenates and found Syt4 was not enriched in the SV fraction. Syb2, a known SV protein was used as a control for this experiment and was highly enriched in SV fractions (Fig 3.1 D). Furthermore, in immuno-organelle isolation from brain homogenate using anti-Syt1 and anti-Syb2 to immuno-isolate SVs, we found Syt4 in the supernatant and not in the 
bound fraction, confirming Syt4s distinct localization compared to SVs proteins (Fig 3.1 E).

All biochemistry experiments were done with Dr. Saheeb Ahmed (a postdoc in our lab). Together, these immunocytochemistry and biochemistry experiments show that Syt4 localises to DCVs, and not to SVs.

A

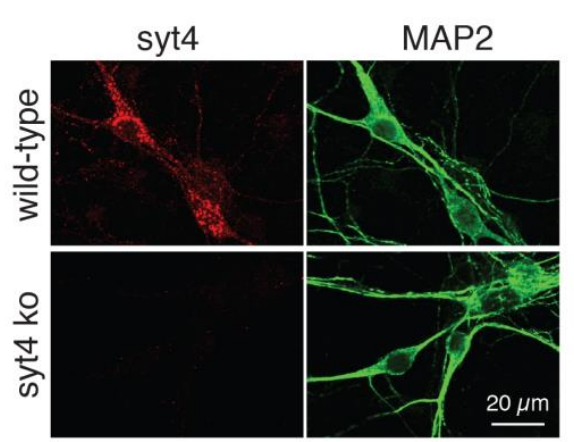

C

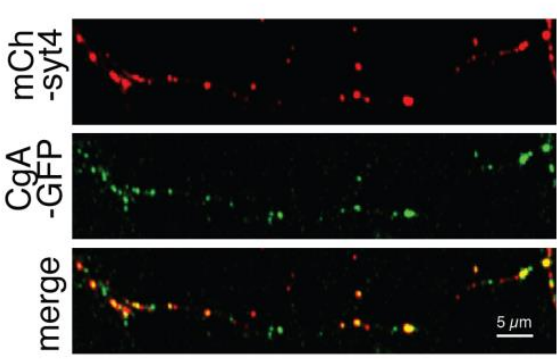

B
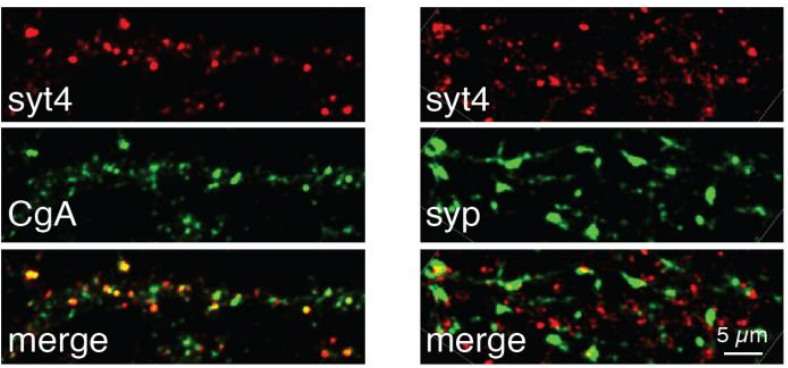

$\mathrm{D}$

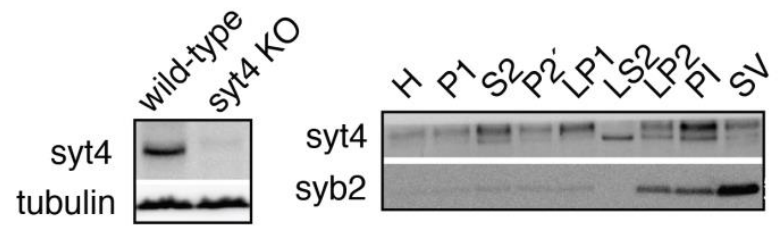

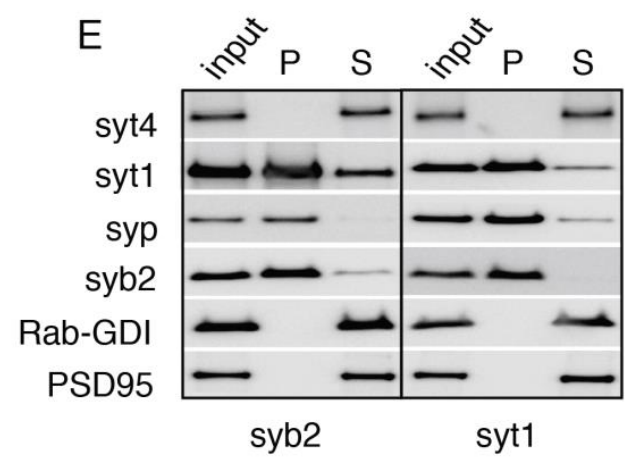

Figure 3.1 Syt4 is localized to DCVs

(A) Example images of hippocampal mouse neurons from wild-type and Syt4 knockouts (Syt4 KO) immunostained for endogenous Syt4 (red) and Map2 (green), a dendritic marker. Scale bar is $20 \mu \mathrm{m}$. (B) Hippocampal rat neurons (DIV14) stained with Syt4 (red) and CgA (green) a known DCV cargo, or the synaptic vesicle marker, Syp. Scale bar is $5 \mu \mathrm{m}$. C) Images of neurons co-transfected with mCherry-Syt4 (red) and CgAGFP (green) showing high co-localization of Syt4 with CgA. Scale bar is $5 \mu \mathrm{m}$ (D) Syt4 antibody testing in Western blots of wild-type and Syt4 knockout brain homogenates, and its localization in subcellular fractions of brain homogenate. (E) Immuno-organelle isolation of SVs with Syt1 and Syb2 from brain homogenate. Antibodies conjugated to beads are indicated below the blots and antibodies used for Western blotting are indicated to the left. Syt4 in both the blots was seen only in the supernatant confirming it's absence on synaptic vesicles. 


\subsection{DCVs are highly mobile on microtubules in axons}

Unlike SVs, DCVs are synthesized in the cell soma and are then trafficked long-distances along microtubules to release sites in axons (Scalettar, 2006). We verified that Syt4 vesicles were also highly mobile (Fig 3.2 A). To confirm the trafficking of DCVs on microtubules, hippocampal neurons transfected with mCherry-Syt4 were treated with $10 \mu \mathrm{M}$ nocodazole, a microtubule dissociating drug, for 30 minutes prior to imaging (Eilers et al., 1989; Colin et al., 2008). We observed a significant decrease in vesicle displacement/sec and also in the mobile vesicle percentage following this treatment (Fig 3.2 B). In control mCherry-Syt4 transfected axons, displacement/s was $0.07 \pm 0.0018 \mu \mathrm{m} / \mathrm{s}$ whereas in nocodazole treated cultures it was significantly decreased to $0.038 \pm 0.0011 \mu \mathrm{m} / \mathrm{s}$ ( $n=4950$ and 4766 vesicles for control and nocodazole treated from 3 different cultures). Similarly, the mobile vesicle percentage of control was $19.7 \pm 2.15 \%$ and of nocodazole treated was $11.4 \pm 2.2 \%$ of vesicles $(n=13$ and 13 videos of control and nocodazole treated from 3 different experiments, respectively). This decrease in displacement and mobility of Syt4containing vesicles on treatment with nocodazole indicates that Syt4 vesicles move along microtubules in axons.

At the Drosophila NMJ, long-range trafficking of DCVs shows a circuitous trafficking pattern termed as "sushi-model". In this model, DCVs filled with cargoes are routed to go first from the cell soma to the very distal end of axon where they switch directions and are then actively circulated retrogradely to ensure an even distribution of DCVs at all synapses (Moughamian and Holzbaur, 2012; Wong et al., 2012). To determine if this property of DCVs is also true in hippocampal neurons, we scanned $\sim 5 \mathrm{~mm}$ of mid-axonal processes in hippocampal neurons and observed 992 vesicles. We found that in mid-axon regions $62.5 \%$ of vesicles were stationary, $37.2 \%$ of vesicles moved unidirectionally (either in the anterograde or retrograde direction) and interestingly, only a very minimal fraction of around $0.3 \%$ of vesicles switched direction in mid axon regions (Fig $3.2 \mathrm{C}$ ). 
A

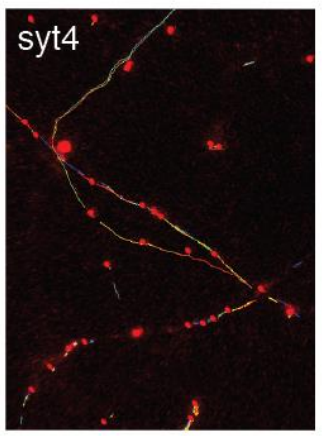

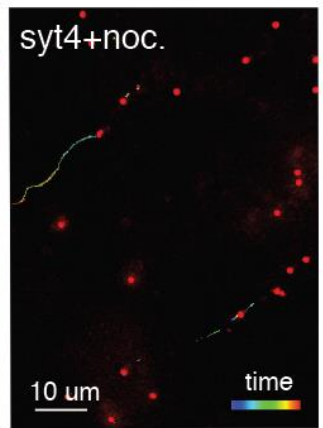

$\mathrm{B}$
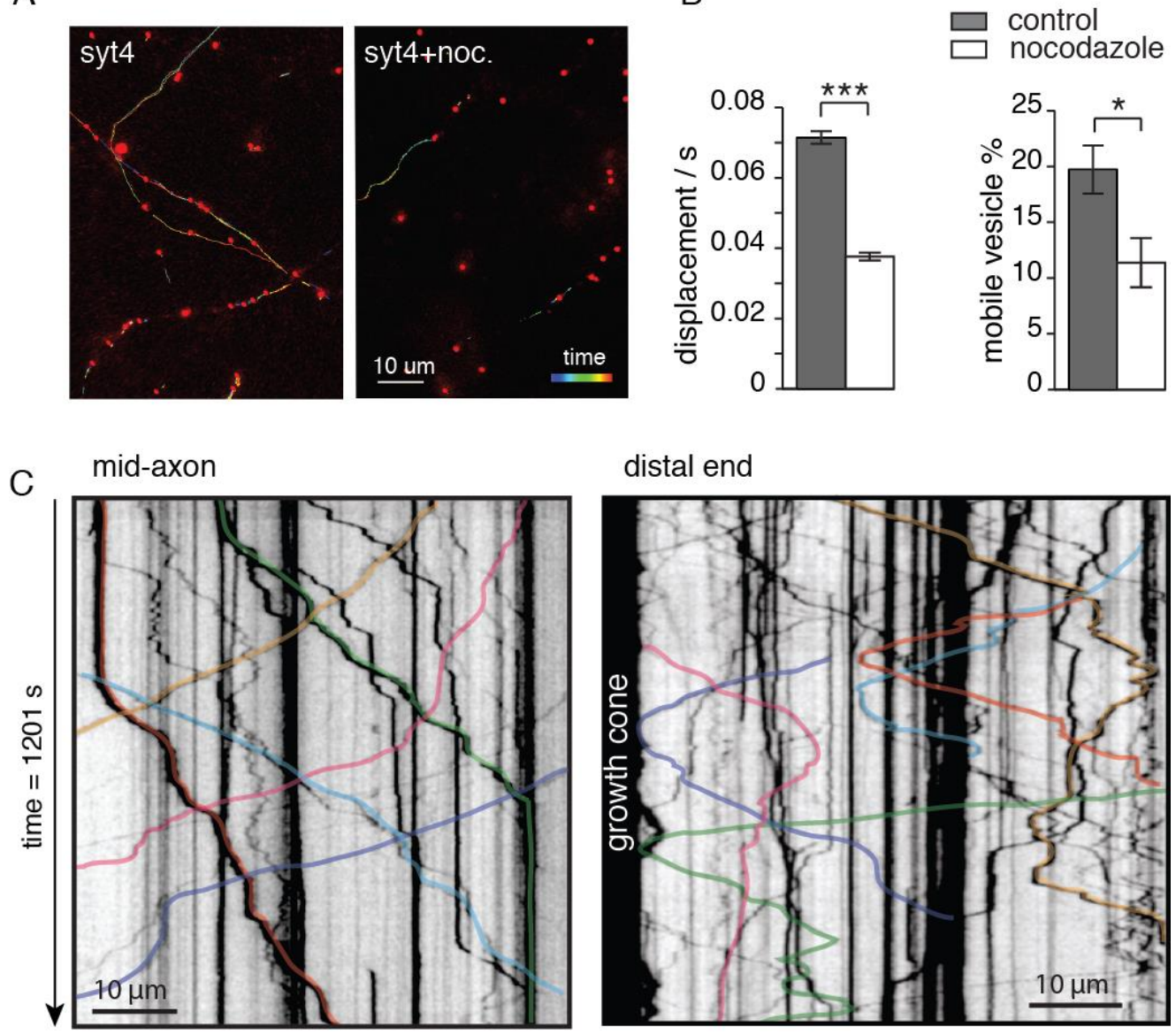

distal end

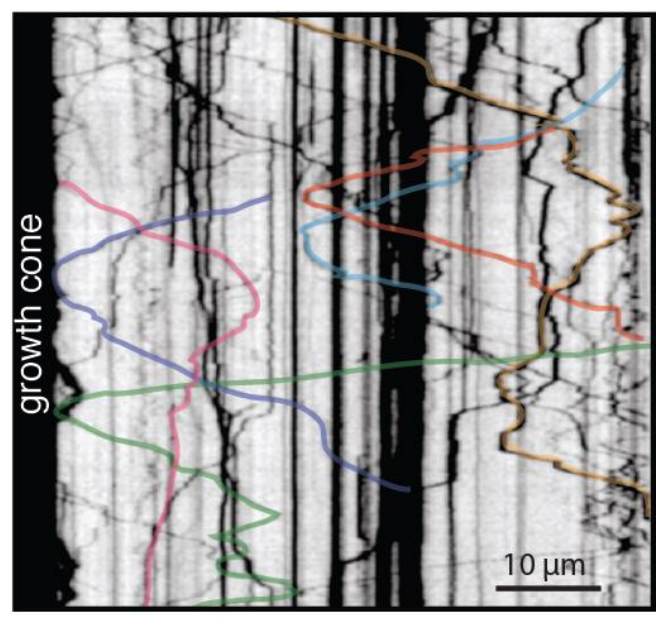

D

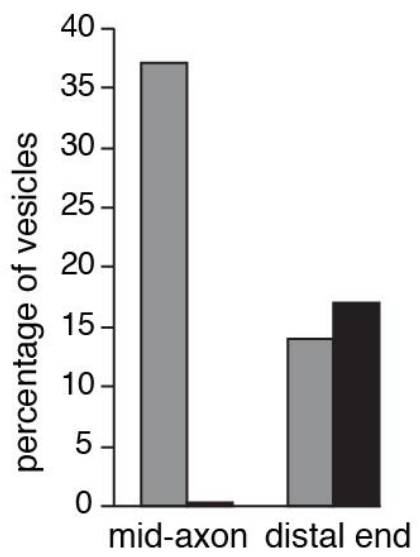

口 unidirectional

- switching directions

Figure 3.2 DCVs undergo long-range trafficking on microtubules in axon.

(A) Snapshots of trafficking of Syt4 vesicles in control and nocodazole treated mCherry-Syt4 transfected cultures. Scale bar is $10 \mu \mathrm{m}$. (B) Quantification of displacement/s and mobile vesicle percentage of the two conditions indicating that Syt4 mobility is microtubule dependent $(n=4950$ and 4766 vesicles for displacement/s and for mobile percentage $n=13$ and 13 videos of control and nocodazole treated from 3 different experiments, respectively). (C) Kymographs showing long-range trafficking of Syt4 vesicles in midaxon regions and distal ends of axons (where the black region is the growth cone). Colored trajectories in kymographs show the unidirectional or switching directions of vesicles. (D) Quantified percentage of vesicles in mid-axon and distal ends of axons shows mainly unidirectional movement in mid-axon regions and a higher percentage of switching in distal ends near the growth cone in hippocampal neurons. (Total length of axon scanned for mid-axon $=4948.16 \mu \mathrm{m}$ and total number of vesicles $=992$, total length of distal axon/growth cone regions examined $=1,008 \mu \mathrm{m}$ and total number of vesicles $=200$ ). Scale bar is $10 \mu \mathrm{m}$. Error indicates S.E.M. Significance was determined by Student's t-tests with Bonferroni correction $\left(^{*} p<0.05\right.$ and $* * *$ $\mathrm{p}<0.001)$. 
On the other hand, in distal axonal ends specifically near growth cone regions, we examined 200 vesicles in $1,008 \mu \mathrm{m}$ of regions and found that a comparatively large fraction of vesicles (17\%) switch directions (Fig 3.2 D). DCVs were seen switching direction along a relatively large region within approximately $100 \mu \mathrm{m}$ of the growth cone and not exactly at the very end, thus the vesicles defined as unidirectional near the growth cone may have switched directions outside the field of view. These data suggest that DCVs in hippocampal neurons also follow the "sushi-model" mode of trafficking. They are routed circuitously, from the soma to the distal end of axons, where they change direction and traffic back to the soma.

After observing the property of long-range trafficking of Syt4 vesicles, we were interested in knowing whether and how release sites in axons capture these transiting vesicles. Protein kinases have been shown to play an important role in regulating the transport of cargoes by either phosphorylating motor proteins, adaptor proteins, cargoes or by modifying microtubule networks (Mcllvain et al., 1994; Kumar et al., 2000; Gibbs et al., 2015). Syt4 is phosphorylated at S135 by JNK, which enhances $\mathrm{Ca}^{2+}$ evoked release in PC12 cells (Mori et al., 2008). Hence, we wanted to test if Syt4 phosphorylation at S135 also affects trafficking, capture and fusion in hippocampal neurons.

\subsection{More number of synapses at DIV13 compared to DIV6}

We used Syt4 phosphorylation mutants; Syt4 S135A (phosphodeficient) and Syt4 S135E (phosphomimetic) compared to control Syt4 to investigate the effects of phosphorylating Syt4 at S135 on the trafficking of DCVs before and after synapse formation. First, we compared DIV6 and DIV13 timepoints for the number of synapses in neurons (Ichikawa et al., 1993; Biffi et al., 2013), since we aimed to test the hypothesis that differences in trafficking of Syt4 phosphomutants may occur developmentally. We stained hippocampal neurons with endogenous Syp to mark pre-synaptic sites, PSD-95 to mark post-synaptic sites and Map2 as dendritic marker at both DIV6 and DIV13. As expected, we found significantly more synapses, identified by puncta containing both pre- and post synaptic marker at DIV13 compared to DIV6 (Fig 3.3 A and B). 
A DIV 6 - MAP2 / PSD95 / syp DIV 13 - MAP2 / PSD95 / syp

B
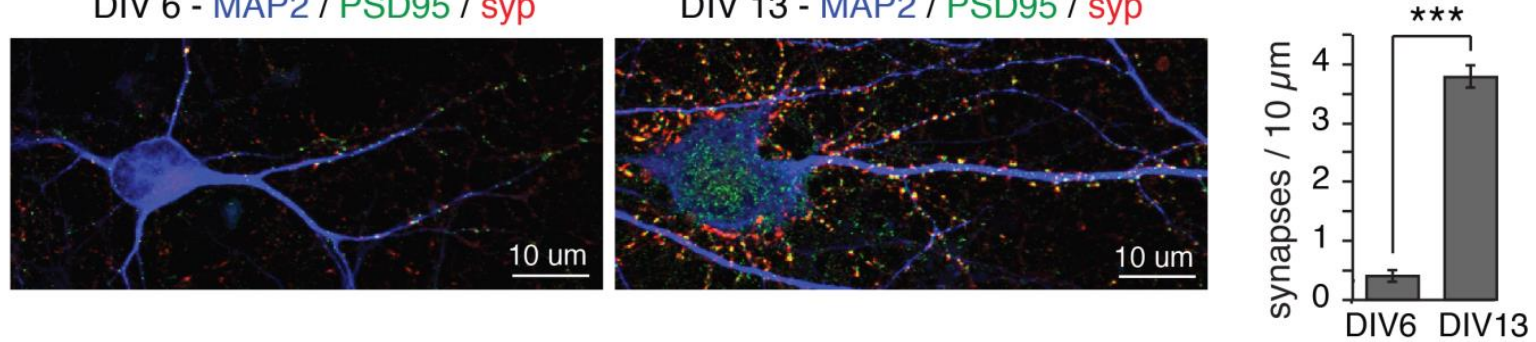

Figure 3.3 Comparison of synapses at DIV 6 and DIV 13

(A) Immunofluorescence images of hippocampal neurons at DIV6 and DIV13 stained with MAP2, PSD-95 and Syp to mark dendrites, postsynaptic and presynaptic sites, respectively. Scale bar is $10 \mu \mathrm{m}$. (B) Quantification of the number of synapses at DIV6 and DIV13 showing significantly more synapses/10 um at DIV13 than DIV6 ( $n=17$ images each of DIV6 and DIV13).

\subsection{Phosphorylating Syt4 at S135 affects DCVs trafficking}

We then aimed to test the effect of phosphorylation of Syt4 at S135 on vesicle trafficking before synapse formation, DIV6. Hippocampal neurons were transfected at DIV3 with mCherry-tagged control, S135A or S135E Syt4 and vesicle trafficking was assessed by live cell imaging at DIV6. We found that all three Syt4 control, Syt4 S135A and Syt4 S135E were highly mobile at DIV6 after visualized by color-coded tracks in Imaris and in kymographs (Fig 3.4 A top and bottom panels). We quantified the population dynamics of vesicle trafficking by analyzing several parameters including average vesicle speed, displacement and mobile vesicle percentage using Imaris and a customized Matlab code.

Frequency distribution and quantification of average speed $(\mu \mathrm{m} / \mathrm{s})$ showed that control Syt4 vesicles had an average speed of $0.135 \pm 0.0021 \mu \mathrm{m} / \mathrm{s}$, S135A of $0.119 \pm 0.0018 \mu \mathrm{m} / \mathrm{s}$ and S135E of $0.124 \pm 0.0015 \mu \mathrm{m} / \mathrm{s}$ (Fig 3.4 B and C). Because speed does not measure translocation (i.e. a vesicle could have a high speed but move only back and forth a small distance) we also calculated displacement/s of the vesicle population. Calculated displacement/s of control, S135A and S135E vesicles were $0.056 \pm 0.0018 \mu \mathrm{m} / \mathrm{s}, 0.049 \pm$ $0.0016 \mu \mathrm{m} / \mathrm{s}$ and $0.051 \pm 0.0013 \mu \mathrm{m} / \mathrm{s}$ respectively (Fig 3.4 D) $(n=3703,4619$ and 3937 vesicles for average speed and displacement/s, from 3 different cultures). In brief, there was no significant difference in average speed and displacement between phosphodeficient and phosphomimetic Syt4 vesicles at DIV6, whereas control Syt4 had a higher speed and displacement compared to the mutants. 
A
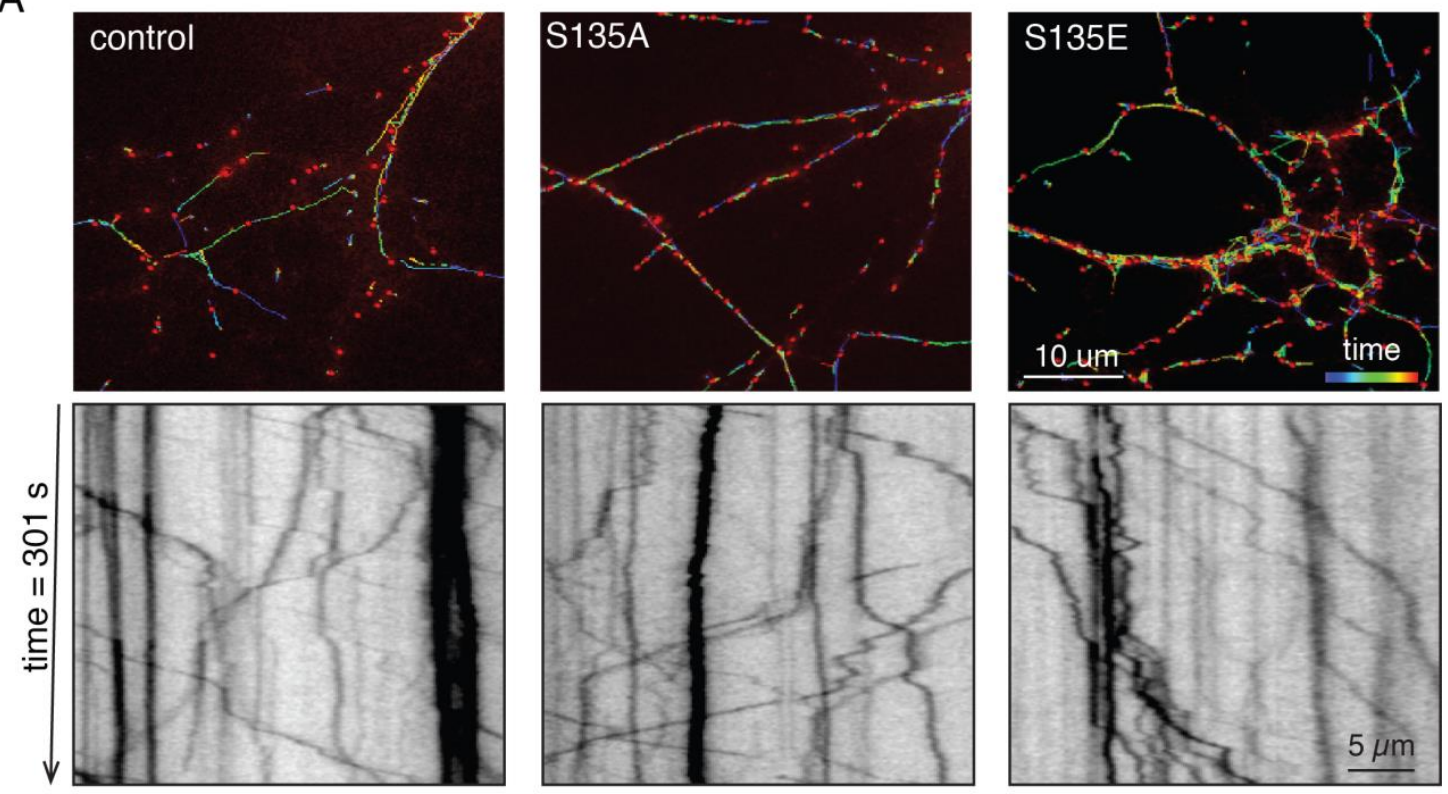

B

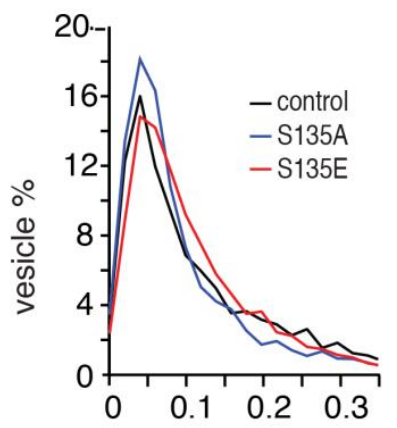

avg. speed $(\mu \mathrm{m} / \mathrm{s})$

D

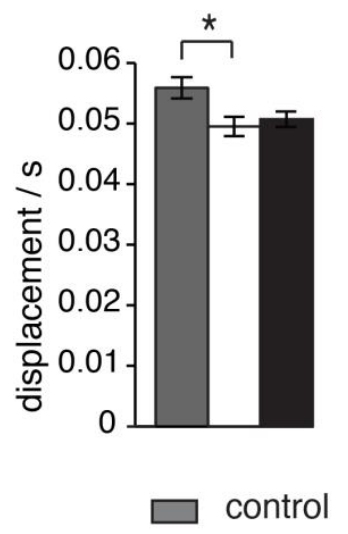

C

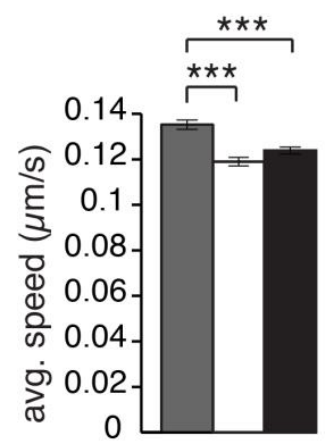

E

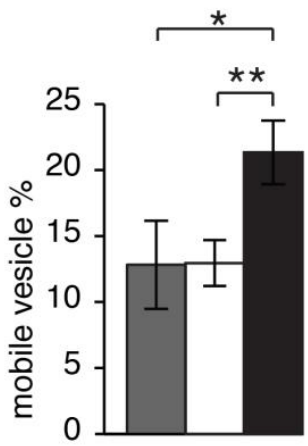

S135E

Figure 3.4 Phosphorylating Syt4 at S135 increases DCV mobility before synapse formation at DIV6

(A) Immunofluorescence images of hippocampal neurons at DIV6 and DIV13 immunostained with MAP2, PSD-95 and Syp to mark dendrites, postsynaptic and presynaptic sides respectively. Scale bar is $10 \mu \mathrm{m}$. (B) Quantification of number of synapses at DIV6 and DIV13 showing significantly more number of synapses/10 $\mu \mathrm{m}$ at DIV13. (C) Snapshots of trafficking videos and kymographs of control, S135A and S135E transfected hippocampal neurons at DIV6 imaged for $5 \mathrm{~min}(301 \mathrm{~s})$. Scale bar of snapshot is $10 \mu \mathrm{m}$ and in kymographs is $5 \mu \mathrm{m}$. (D) Comparison of frequency distribution of average speed of vesicles (um/s) between control, S135A and S135E. (E) Quantification of average speed $(\mu \mathrm{m} / \mathrm{s})$, displacement $/ \mathrm{s}(\mathrm{F})$ and mobile vesicle percentage $(\mathrm{G})$ indicating that control Syt4 vesicles have significantly higher speed compared to S135A and S135E vesicles. 
Also, displacement/s of control vesicles was slightly more than that of S135A. The mobile vesicle percentage of S135E was significantly higher compared to control and S135A ( $n=3703,4619$ and 3937 vesicles for average speed and displacement/s and for mobile percentage, $n=11,13$ and 12 videos of control, S135A and S135E from 3 different cultures). Error indicates S.E.M. Significance was determined by Student's t-tests with Bonferroni correction $\left({ }^{*} p<0.05,{ }^{*} p<0.01\right.$ and $\left.* * * p<0.001\right)$.

Interestingly, the mobile vesicle percentage (defined as vesicles with a displacement of at least $1 \mu \mathrm{m}$ over the duration of each 5 minute timelapse) of S135E vesicles was significantly higher than control and S135A vesicles. Mobile vesicle percentage of control, S135A and S135E vesicles were $12.8 \pm 3.35 \%, 12.95 \pm 1.74 \%$ and $21.33 \pm 2.41 \%$, respectively (Fig 3.4 E) ( $n=11,13$ and 12 videos for control, S135A and S135E, respectively, from 3 different cultures). Together, these data indicate that average speed and displacement/s of phosphomutants (S135A and S135E) vesicles were not significantly different before synapse formation. But interestingly, phosphorylation of Syt4 at S135 increases the mobile vesicle percentage of DCVs at DIV 6.

\subsection{Phosphorylating Syt4 at S135 changes DCV mobility at DIV13}

Next, we investigated if trafficking of Syt4 phosphomutants changes at DIV13 after synapse formation. We transfected hippocampal neurons with all three constructs - control, phophodeficient and phosphomimetic Syt4 at DIV 10 and imaged vesicle movement at DIV13. By live cell imaging, we found that all three mutants were still mobile, although interestingly at DIV13, Syt4 control and S135E mobility was reduced compared to their respective mobility at DIV6 analyzed both by Imaris color-coded tracks and by kymographs (Fig $3.5 \mathrm{~A}$ ). The frequency distribution of average speed $(\mu \mathrm{m} / \mathrm{s})$ revealed that S135A had more high moving vesicles whereas S135E had more slow speed vesicles (Fig 3.5 B). Compared to DIV6, average speed of control and S135E vesicles at DIV13 were decreased to $0.093 \pm 0.0019 \mu \mathrm{m} / \mathrm{s}$ and $0.086 \pm 0.0018 \mu \mathrm{m} / \mathrm{s}$ whereas S135A was moving with almost the same speed of $0.114 \pm 0.0021 \mu \mathrm{m} / \mathrm{s}$ (Fig 3.5 C). Vesicle displacement/s also showed the same trend; control and S135E were reduced to $0.036 \pm 0.0016 \mu \mathrm{m} / \mathrm{s}$ and $0.030 \pm 0.0014$ $\mu \mathrm{m} / \mathrm{s}$ at DIV13 whereas S135A diplacement/s remained similar to DIV6, at $0.054 \pm 0.0020$ $\mu \mathrm{m} / \mathrm{s}$ (Fig 3.5 D) ( $\mathrm{n}=2686,2965$ and 3189 vesicles for average speed and displacement/s of control, S135A and S135E, respectively). In summary, while S135E and control vesicles were less mobile after synapse formation, S135A vesicles were as mobile as at DIV6, and there was no change in the speed and displacement of this phosphodeficient mutant at DIV13. 
A
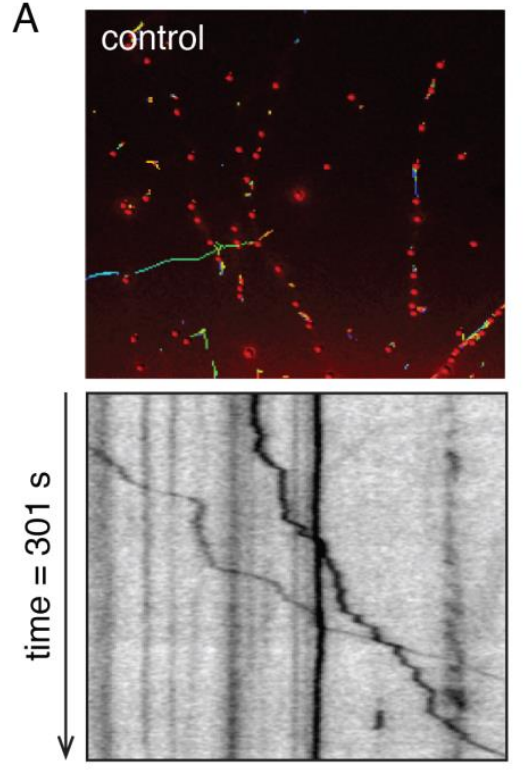

B

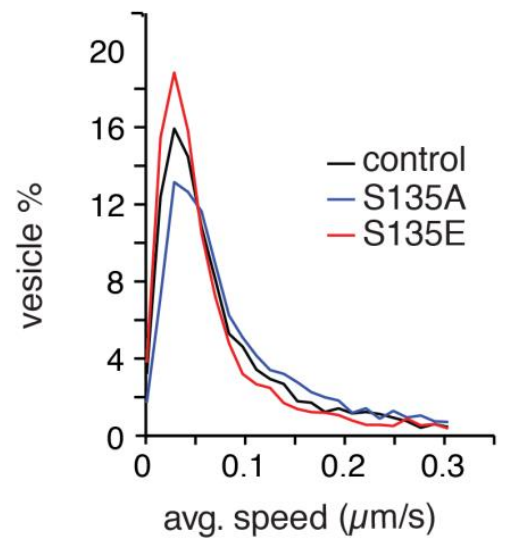

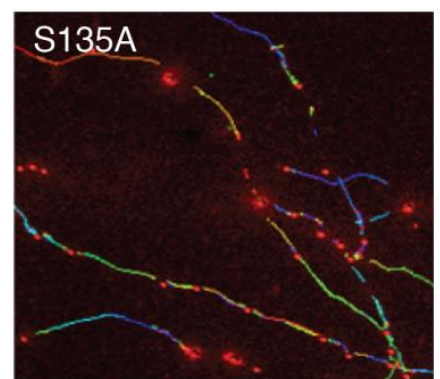

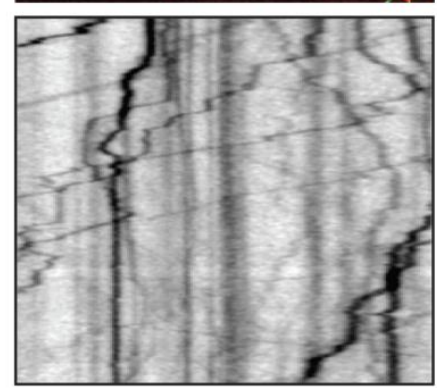

C

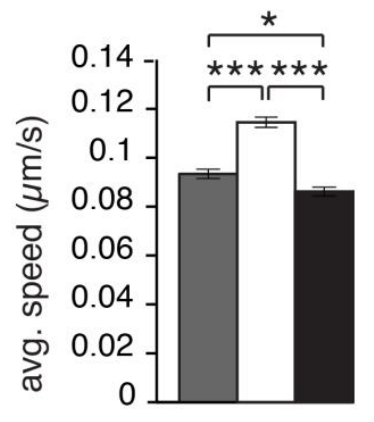

E

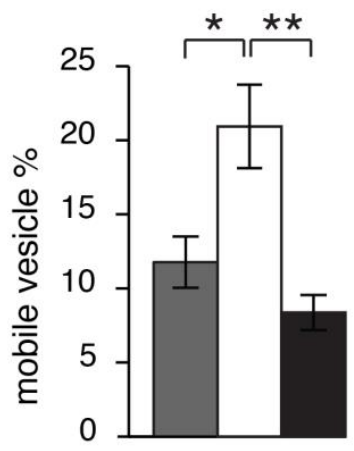

S135E

\section{Figure 3.5 Synapse maturation changes DCVs mobility}

(A) Snapshots of trafficking videos and kymographs of control, S135A and S135E transfected hippocampal neurons at DIV13 imaged for $5 \mathrm{~min}(301 \mathrm{~s})$. (B) Frequency distribution of average speed $(\mu \mathrm{m} / \mathrm{s})$ of control, S135A and S135E vesicles at DIV13 showed that S135A Syt4 vesicles had a higher mobile percentage with higher velocity. (C) With synapse maturation at DIV13, average speed and displacement/ S (D) of S135A remained similar to DIV6 but average speed and displacement of control and S135E decreased significantly. Also, avg. speed of S135E vesicles was the lowest ( $n=2686,2965$ and 3189 vesicles for average speed and 
displacement/s of control, S135A and S135E, respectively). (E) Quantified mobile vesicle percentage showed that S135A vesicles were highly mobile and S135E were least mobile (for mobile percentage, $n=12,11$ and 14 videos of control, S135A and S135E, respectively from 3 different cultures). Scale of fluorescent images is $10 \mu \mathrm{m}$ and of kymographs is $5 \mu \mathrm{m}$. Error indicates S.E.M. Significance was determined by Student's t-tests with Bonferroni correction ( $* p<0.05, * * p<0.01$ and $\left.{ }^{* * *} p<0.001\right)$.

Surprisingly, we found that the mobile vesicle percentage of S135E, which was highest in DIV6, was lowest at DIV13. It was reduced to $8.37 \pm 1.19 \%$ of vesicles. S135A had $20.9 \pm$ $2.81 \%$ mobile vesicles whereas control remained similar, at $11.8 \pm 1.73 \%$ mobile vesicles (Fig 3.5 E) (For mobile percentage, $n=12,11$ and 14 videos of control, S135A and S135E, respectively, from 3 different cultures). These trafficking experiments showed that with synapse formation, there is a significant decrease in mobility of phosphorylated vesicles, whereas phosphodeficient vesicles show similar trafficking at DIV13 and DIV6. Also, S135E vesicles that were highly mobile at DIV6 were now least mobile at DIV13, whereas S135A vesicles had a significantly higher mobile vesicle percentage at DIV13 than DIV6. This could indicate that phosphorylation of Syt4 is important for reducing the trafficking of DCVs and aiding in capture of DCVs at release sites in mature cultures. 


\subsection{S135E vesicles are least mobile and S135A vesicles are highly mobile in axons}

Since DCVs are known to be more mobile in axons compared to dendrites (de Wit et al., 2006), we examined if the phosphorylation effect on trafficking of DCVs was different in axons and dendrites and if it was more prominent in axons at DIV13. We co-transfected hippocampal neurons at DIV10 with mCherry-tagged control or mCherry-tagged Syt4 phosphomutants and EGFP (a neuronal volumetric marker). We distinguished axons and dendrites in the culture based on morphology marked by GFP. Dendrites had a larger diameter and were decorated by dendritic spines, and were branched at angles less than ninety degrees within approximately $100 \mu \mathrm{m}$ of the cell soma, whereas axons were much thinner and could be identified as the only process that projected far away from the cell body. After identifying these regions, we performed time lapse imaging to visualize the differences in vesicle trafficking of phosphorylation mutants of Syt4 in axons and dendrites specifically.

In axons, we observed by Imaris and kymographs that S135A vesicles were the most mobile

(Fig 3.6 A). Frequency distribution and average speed quantification showed that S135E vesicles were the least mobile with an average speed of $0.113 \pm 0.0017 \mu \mathrm{m} / \mathrm{s}$ for S135A and $0.123 \pm 0.0018 \mu \mathrm{m} / \mathrm{s}$ for control (Fig 3.6 B, C). Vesicle displacement/s also showed that S135A and control covered more distance compared to S135E (displacement/s of control, S135A and S135E was $0.058 \pm 0.0017 \mu \mathrm{m} / \mathrm{s}, 0.075 \pm 0.0017 \mu \mathrm{m} / \mathrm{s}$ and $0.049 \pm 0.0015 \mu \mathrm{m} / \mathrm{s}$ respectively (Fig 3.6 D). The percentage of S135E mobile vesicles was significantly less than control and S135A (mobile vesicle percentage of control, S135A and S135E were $17.1 \pm$ $1.51 \%, 20.9 \pm 1.43 \%$ and $11.6 \pm 1.49 \%$, respectively) (Fig $3.6 \mathrm{E}$ ). These results indicate that in axons at DIV13, phosphodeficient vesicles are highly mobile and phosphomimetic vesicles are least mobile. Thus phosphorylation of Syt4 at S135 decreases DCVs mobility. 


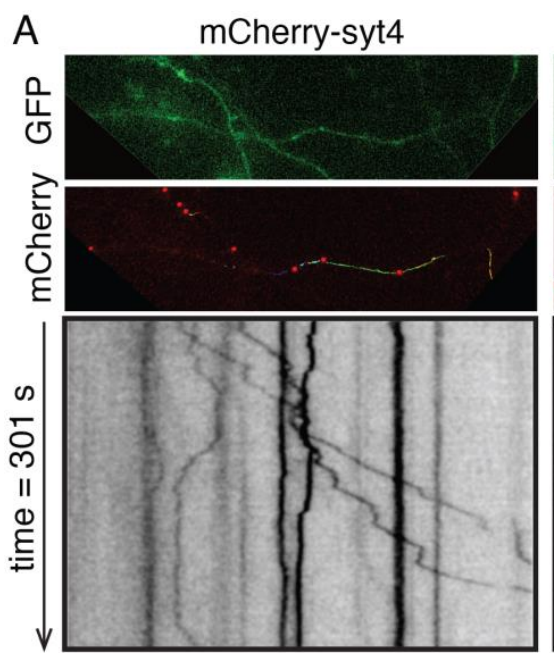

B

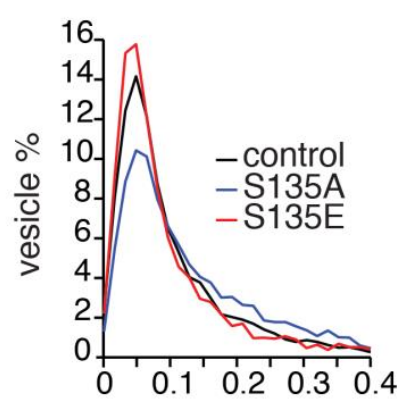

avg. speed $(\mu \mathrm{m} / \mathrm{s})$
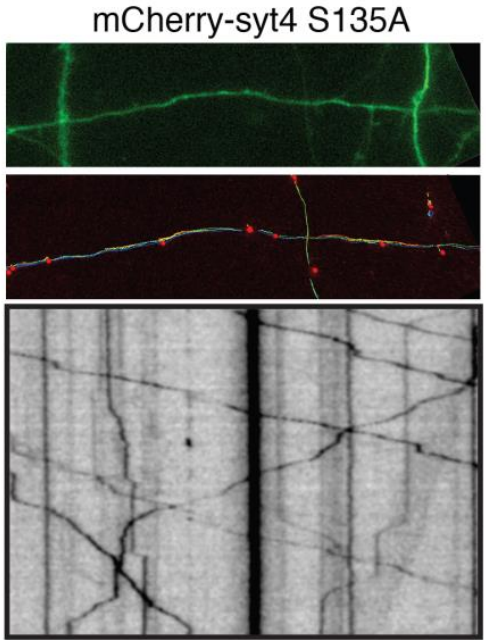

C

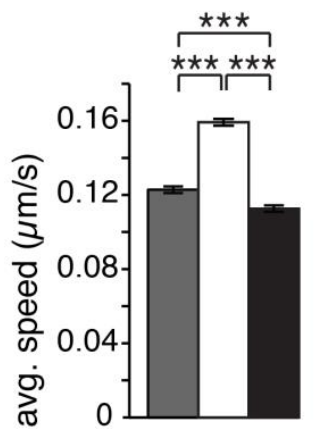

F

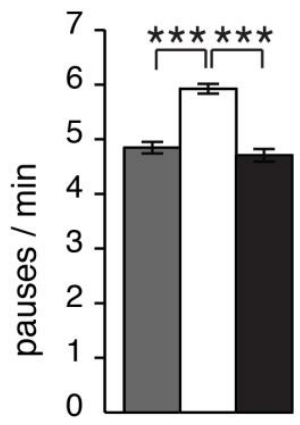

mCherry-syt4 S135E
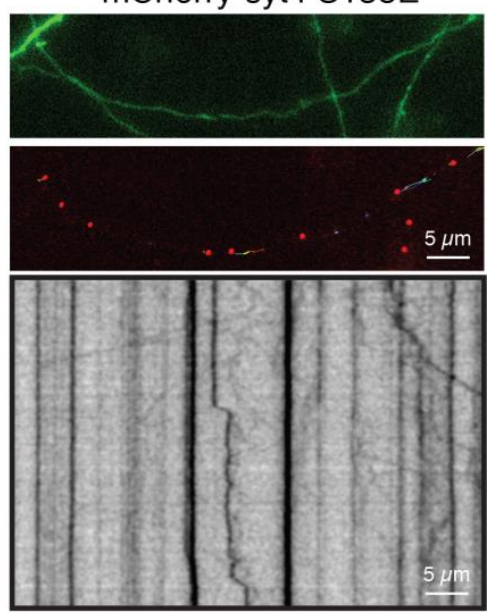

D

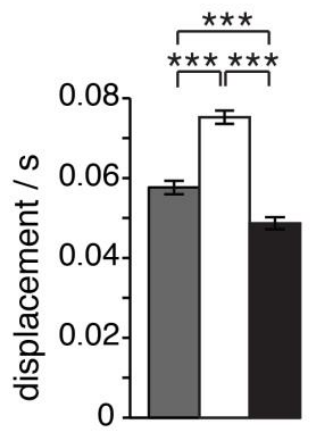

G

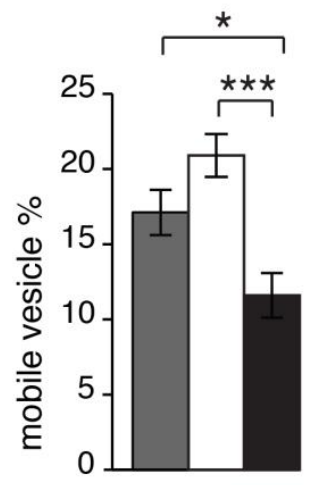

control

S135A

S135E

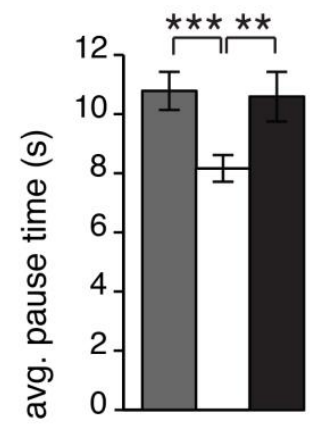

Figure 3.6 Syt4 vesicles are highly mobile in axons at DIV13. S135E vesicles are least mobile with decreased speed and displacement/s compared to control and S135A vesicles.

Hippocampal neurons were co-transfected with eGFP and mCherry-Syt4, mCherry-syt4 S135A or mCherrySyt4 S135E. Axonal and dendritic regions were chosen based on morphology marked by eGFP and then time lapse images were taken for $5 \mathrm{~min}$ (301 s) in the mCherry channel. (A) Images indicating axonal regions marked with eGFP, snapshots from the trafficking videos of these selected regions and respective kymographs of control, S135A and S135E shows the difference in trafficking of DCVs in axons at DIV13. (B) Frequency distribution of average speed $(\mu \mathrm{m} / \mathrm{s})$ of control, S135A and S135E indicating that S135A has more vesicles with higher avg. speed. (C) Quantified avg. speed and displacement/s (D) showed that S135A vesicles move with significantly higher speed and were cover larger distances compared to control and S135E. S135E 
vesicles move with the least speed and covered significantly less distance. (E) Mobile vesicle percentage indicates that S135A vesicles were highly mobile and S135E were least mobile. (F) Quantification of pauses/min shows that S135A vesicles have a higher number of pauses/min compared to control and S135E. (G) Average pause time of S135A vesicles was significantly less than control and S135E vesicles ( $n=4476,6502$ and 4228 vesicles for average speed, displacement/s, pauses/min and avg. pause time of control, S135A and S135E, whereas for mobile percentage $n=27,31$ and 32 videos, respectively from 4 different cultures). Scale bar of fluorescent images and of kymographs is $5 \mu \mathrm{m}$. Error indicates S.E.M. Significance was determined by Student's t-tests with Bonferroni correction ( ${ }^{*} p<0.05, * * p<0.01$ and $\left.* * * p<0.001\right)$.

Consequently, we examined the possible reason for this effect. We investigated pauses/min and average pause time of vesicles in each condition, since decreased mobility could be due to either an increased number of pauses or increased duration of pause time. We found that the number of pauses/ min was surprisingly more for highly mobile S135A vesicles compared to S135E and control (pauses/min of control, S1135A and S135E were $4.85 \pm 0.11,5.92 \pm 0.09$, and $4.71 \pm 0.12$, respectively (Fig 3.6 F). One would expect the number of pauses per min to be less if vesicles are highly mobile, which was not the case in our pause analysis. Then, we examined the average pause time of these vesicles and, indeed, the average pause time of S135A was the least. Average pause time of control, S135A and S135E vesicles were $10.79 \pm 0.64 \mathrm{~s}, 8.16 \pm 0.45 \mathrm{~s}$ and $10.59 \pm 0.84 \mathrm{~s}$, respectively

(Fig 3.6 G). This suggests that because phosphodeficient vesicles traffic the most, they scan more "capture/release sites" resulting in a higher frequency of pauses, but the pause duration at these sites are not that long, unlike phosphomimetic vesicles and control vesicles, which have less pauses but longer pause duration $(n=4476,6502$ and 4228 vesicles for average speed, displacement/s, pauses/min and avg. pause time of control, S135A and S135E vesicles, respectivley and for mobile percentage $n=27,31$ and 32 videos, respectively, from 4 different cultures).

\subsection{Syt4 vesicles are comparatively less mobile in dendrites}

We then observed the trafficking of Syt4 vesicles in dendrites and the effect of phosphorylating Syt4 at S135. We found that the mobility of vesicles in dendrites was much less than in axons for control and phosphomutant vesicles analysed both by Imaris and kymographs (Fig 3.7 A). But we observed the same phosphorylation effect on average speed and displacement/s in dendrites as in axons. S135A vesicles were most mobile covering more distance compared to S135E and control vesicles (avg. speed of control, S135A and S135E were $0.058 \pm 0.0016 \mu \mathrm{m} / \mathrm{s}, 0.068 \pm 0.0024 \mu \mathrm{m} / \mathrm{s}$ and $0.059 \pm 0.0017 \mu \mathrm{m} / \mathrm{s}$, 
whereas displacement/s was $0.017 \pm 0.0013 \mu \mathrm{m} / \mathrm{s}, 0.028 \pm 0.0021 \mu \mathrm{m} / \mathrm{s}$ and $0.018 \pm 0.0014$ $\mu \mathrm{m} / \mathrm{s}$, respectively) (Fig 3.7 B, C and D). Mobile percentage of control and both mutants was significantly less (<5\%) compared to axons (Fig 3.7 E) ( $n=1319,1489$ and 1088 vesicles for average speed, displacement/s of control, S135A and S135E, and for mobile percentage $\mathrm{n}=13,13$ and 14 videos from 4 different cultures). Together, these experiments are consistent with the literature that DCVs are more mobile in axons than in dendrites (Adachi et al., 2005; de Wit et al., 2006). In addition, we showed that S135E vesicles are least mobile compared to control and S135A vesicles at DIV 13.

A
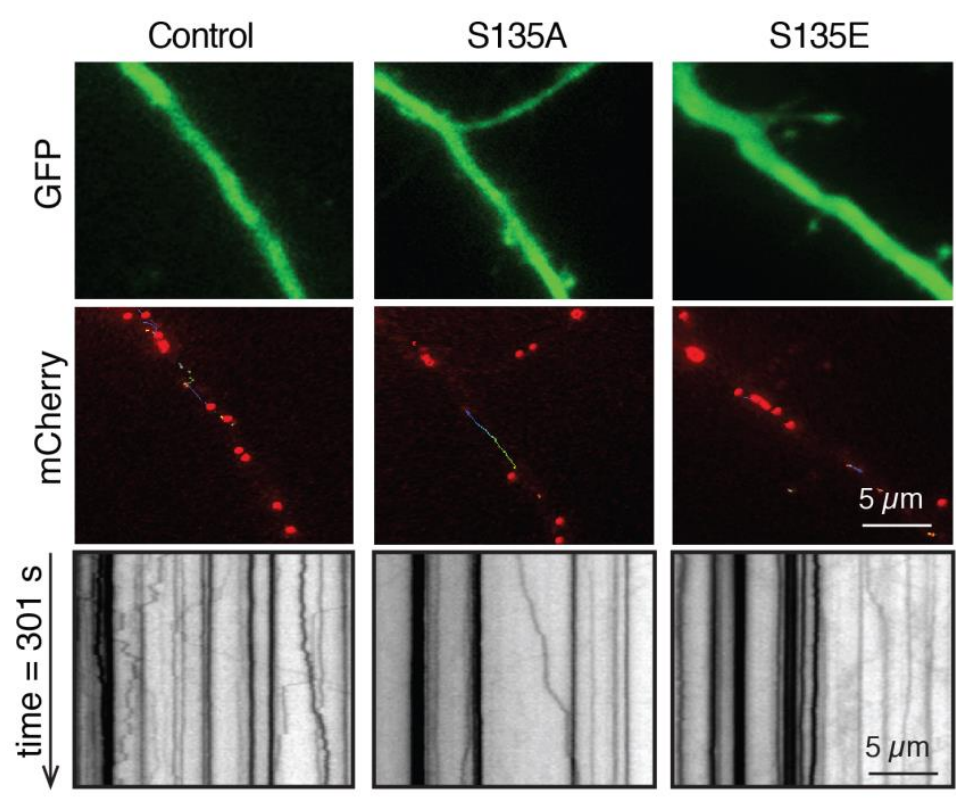

B

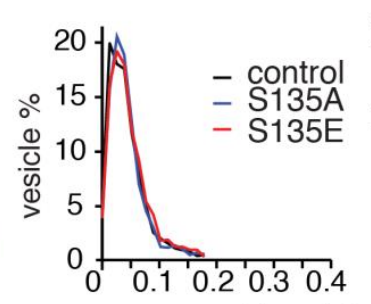
avg. speed $(\mu \mathrm{m} / \mathrm{s})$

$D$

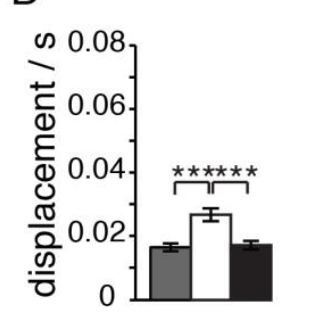

$\square$ control $\square \mathrm{S} 135 \mathrm{~A}$
C

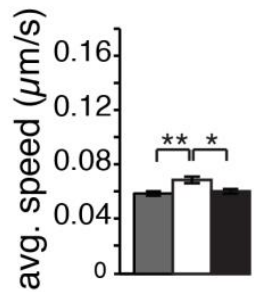

E

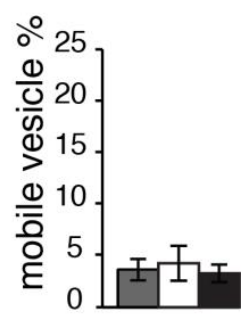

S135E

Figure 3.7 Syt4 vesicles are less mobile in dendrites at DIV13

(A) Images indicating dendritic regions marked with eGFP, snapshots from the trafficking videos of these selected regions and respective kymographs of control, S135A and S135E transfected neurons at DIV13. (B) Comparison of frequency distribution of average speed $(\mu \mathrm{m} / \mathrm{s})$ of control, S135A and S135E vesicles. (C), Quantification of average speed (um/s), displacement/s (D) and mobile vesicle percentage (E). Avg. speed and displacement/s showed the same trend as in axons but with comparatively low values indicating that DCVs are more mobile in axons compared to dendrites. Mobile vesicle percentage was not different in all three conditions in dendrites ( $n=1319,1489$ and 1088 vesicles for average speed and displacement/s of control, S135A and S135E, whereas for mobile percentage $n=13,13$ and 14 videos, respectively, from 4 different cultures). Scale bar of fluorescent images and kymograph is $5 \mu \mathrm{m}$. Error indicates S.E.M. Significance was determined by Student's t-tests with Bonferroni correction ( ${ }^{*} p<0.05,{ }^{* *} p<0.01$ and $\left.{ }^{* * *} p<0.001\right)$. 


\subsection{S135E shows less interaction with Kif1A motor protein}

Kif1A is the primary microtubule dependent motor protein responsible for fast axonal transport of DCVs in hippocampal neurons (Lo et al., 2011) and Syt4 has been reported to bind Kif1A (Arthur et al., 2010). Based on these evidences, we next tested if S135E, the least mobile vesicles, had a difference in interaction with the Kif1A motor protein compared to control and S135A. Differences in binding to motor proteins could possibly explain the decrease in mobility of S135E vesicles. We tested this by performing coimmunoprecipitation (Co-IP) experiments in HEK293 cells co-transfected with Kif1A-GFP and mCherry-tagged Syt4 control, mCherry-Syt4 (S135A) or mCherry-Syt4 (S135E) using GFP-Trap beads (Fig 3.8 A). A specific Kif1A-GFP band around 230 kDa (200 kDa of Kif1A and $27 \mathrm{kDa}$ of GFP) was seen in bound fractions in all three cases. Then, we blotted for Syt4, to check its interaction with Kif1A (Fig 3.8 B). We found that Syt4 control and Syt4 S135A showed a relatively high intensity band around 78 kDa (49 kDa Syt4 and 28.8 kDa mCherry) compared to S135E. The reduced intensity of the Syt4 S135E band indicated a reduced interaction of Syt4 S135E with Kif1A compared to control and S135A. This experiment was done with the help of Dr. Katja Burk (a postdoc in the lab). This result shows that phosphorylation may result in destabilization of motor-microtubule complexes with Syt4, leading to decreased trafficking and increased capture.

A

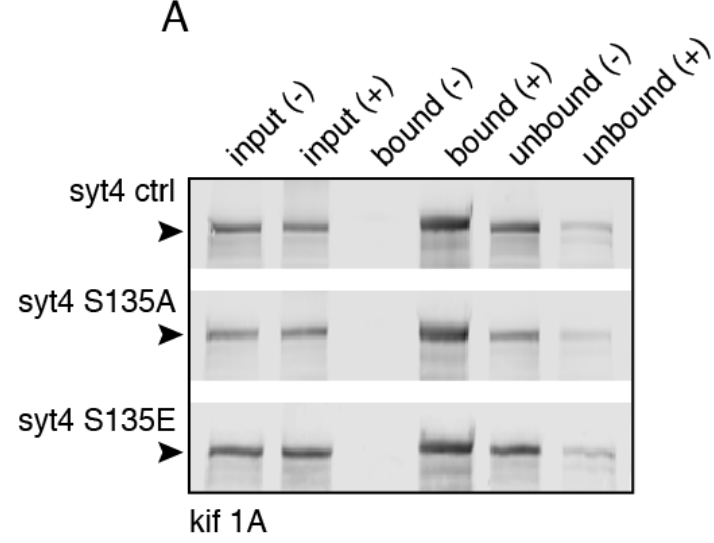

B

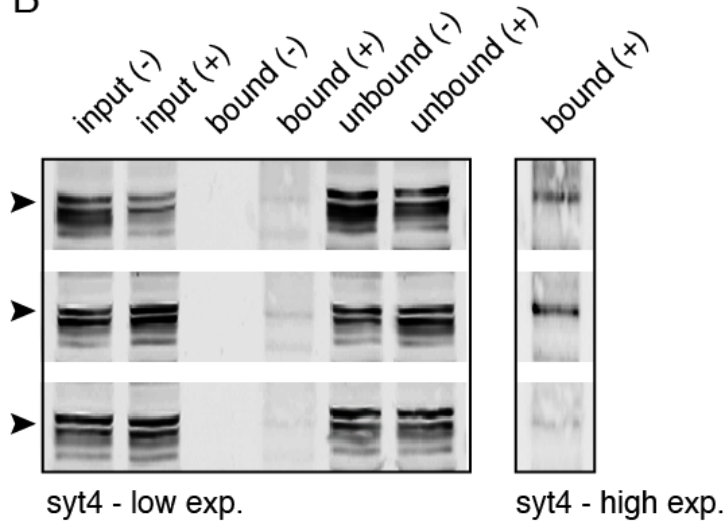

Figure 3.8 Syt4 S135E shows less interaction with Kif1A motor protein

(A) Blots for Kif1A and Syt4 (B) in co-immunoprecipitation experiments of Kif1A-GFP with mCherry-Syt4 control, mCherry-Syt4 S135A or mCherry-Syt4 S135E co-expressed in HEK 293 cells. GFP-Trap beads (marked by plus sign (+)) against the GFP fluorophore of Kif1A were used to co-immunoprecipitate Kif1A and to test and compare the interaction of Kif1A and the phosphomutants. A binding control of agarose beads were used as positive controls marked by minus signs (-). Low and high exposures of Syt4 blots are shown. Input (HEK 
cells transfected lysate), bound and unbound fractions of control or GFP-trap beads are labelled above each lane on the top of the blot. Three conditions were tested: Syt4 (ctrl), Syt4 (S135A) and Syt4 (S135E) which are labelled on the left of blots. Black arrow on left of blots marks correct size bands ( $n=3$ different experiments).

\subsection{Overexpression of Kif1A rescues S135E vesicles mobility}

Since S135E has the least mobility compared to S135A and control, and S135E Syt4 has reduced binding to Kif1A, we tested if over-expression of Kif1A has an effect on trafficking of Syt4 control and phosphomutant vesicles (Wong et al., 2002; Kondo et al., 2012). We cotransfected hippocampal neurons with Kif1A-GFP and control, S135A or S135E mCherrySyt4 at DIV10 and imaged trafficking on DIV13. First, we found co-transport of Kif1A with Syt4 vesicles in all three conditions. The percentage of S135E and S135A vesicles travelling with Kif1A was significantly higher compared to control Syt4 vesicles traveling with Kif1A (percentage of control, S135A and S135E vesicles traveling with Kif1A was $70.4 \pm 6.87 \%$, $85.8 \pm 4.22 \%$ and $86.4 \pm 3.15 \%$, respectively) (Fig 3.9 C). We also quantified percentage of Kif1A travelling with Syt4 vesicles and found a similar trend; Kif1A trafficked significantly more with S135E and S135A vesicles compared to control Syt4 (percentage of Kif1A travelling with control, S135A and S135E vesicles were $61.8 \pm 5.67 \%, 78.1 \pm 5.09 \%$ and 79.2 $\pm 3.96 \%$, respectively) ( $n=7,9$ and 9 videos for control, S135A and S135E with Kif1A cotransport, respectively).

Next, we quantified trafficking parameters for control, S135A and S135E vesicles when we co-over-expressed Kif1A-GFP. We found higher mobility of vesicles in all three conditions compared to conditions without Kif1A-GFP overexpression, both by Imaris and in kymographs. Surprisingly, with Kif1A-GFP overexpression, S135E vesicles became highly mobile (Fig 3.9 A and B). Quantification and comparison of trafficking parameters with and without Kif1A-GFP overexpressed showed significant differences. We found that with Kif1A over expression, average speed ( $\mu \mathrm{m} / \mathrm{s})$ and displacement/s of all three conditions; control, S135A and S135E were significantly higher compared to normal Kif1A levels. We also compared average speed $(\mu \mathrm{m} / \mathrm{s})$ and displacement/s of control, S135A and S135E with Kif1A over-expression and found that S135A and S135E were very similar and significantly higher than control Syt4 with Kif1A overexpression (Fig 3.9 D and E). Then, we investigated mobile vesicle percentage and found that with Kif1A overexpression, S135A and S135E had a significantly higher percentage of mobile vesicles compared to normal Kif1A levels. 
Importantly, there was no difference in trafficking among control, S135A and S135E vesicles with Kif1A over-expression (Fig 3.9 F).
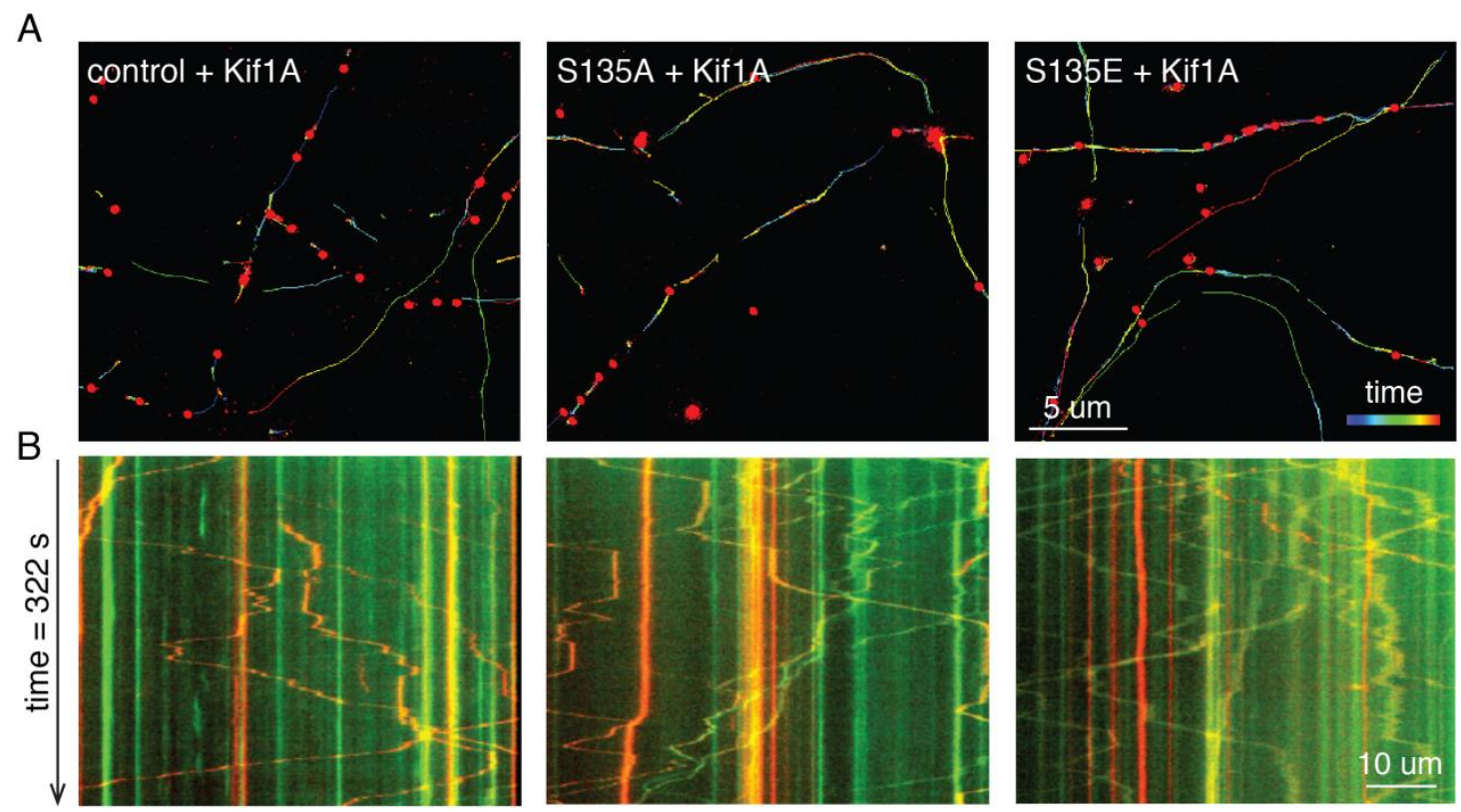

C

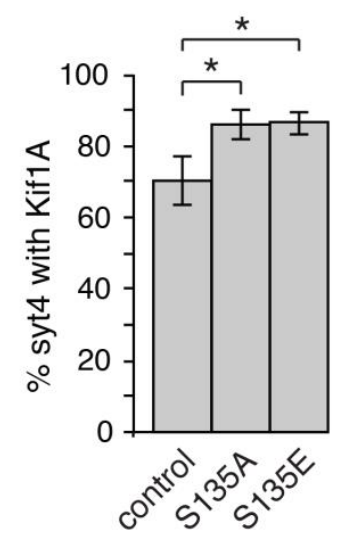

$\mathrm{F}$

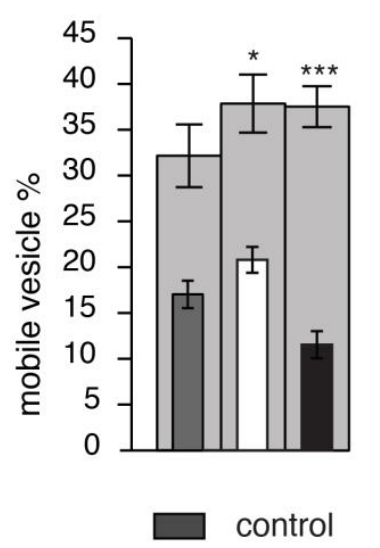

$\mathrm{D}$

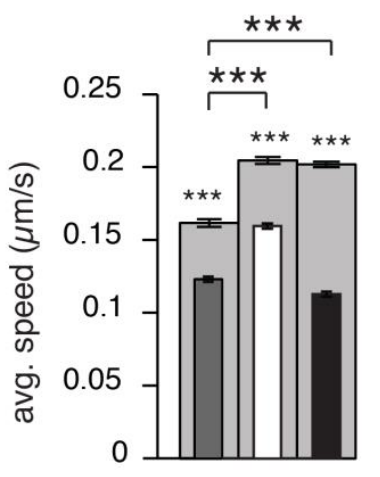

G

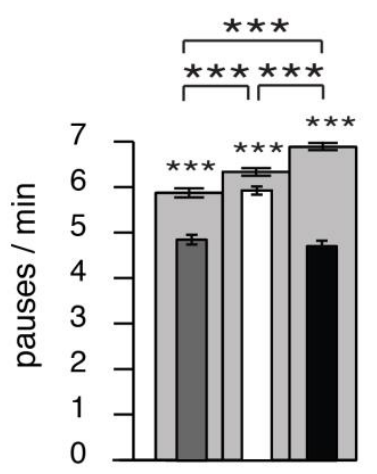

E

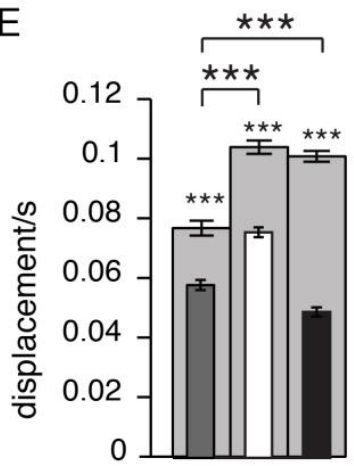

$\mathrm{H}$

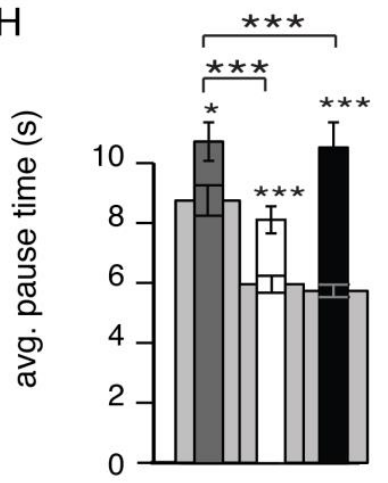

\section{S135A}

S135E

Overexpressed Kif1A

Figure 3.9 Over-expressing Kif1A rescues S135E vesicle mobility

(A) Snapshots of trafficking videos of control, S135A and S135E vesicles when overexpressed with Kif1A-GFP. Scale bar is $5 \mu \mathrm{m}$. (B) Respective kymographs of Syt4 vesicles with Kif1A-GFP overexpression at DIV13 imaged for $322 \mathrm{~s}$. Scale bar is $10 \mu \mathrm{m}$. (C) Quantification of average speed $(\mu \mathrm{m} / \mathrm{s})$ and displacement $/ \mathrm{s}$ (D) of control, S135A and S135E vesicles and with Kif1A-GFP overexpression. Kif1A co-transfection causes a significant 
increase in both average speed and displacement/s compared to single transfection. (E) Percentage of mobile S135A and S135E vesicles with Kif1A co-transfected were significantly higher than in single transfected conditions. (F) Pauses/min of vesicles in Kif1A overexpressed conditions was also significantly higher compared to single transfection of control, S135A and S135E Syt4. (G) Quantification of average pause time showed that Kif1A overexpression reduced the pause duration compared to single transfected conditions ( $n=4278,6325$ and 9507 vesicles for average speed, displacement/s, pauses/min and average pause time, whereas for mobile percentage $n=7,9$ and 12 videos, respectively, from 3 different cultures for Kif1A cotransfected conditions). Error indicates S.E.M. Significance was determined by Student's t-tests with Bonferroni correction $\left({ }^{*} p<0.05, * * p<0.01\right.$ and $\left.* * * p<0.001\right)$.

We also examined the frequency and average pause time of these trafficking vesicles and found that highly mobile vesicles in all conditions had more pauses/min but significantly shorter pause durations consistent with the previous data. Quantification of pauses/min showed that with Kif1A over-expression, the frequency of pauses increases in all three conditions and interestingly, the number of pauses/min was highest in S135E vesicles compared to control and S135A vesicles (Fig 3.9 G). In addition, average pause time decreased significantly in all three conditions compared to normal Kif1A levels indicating that these vesicles with Kif1A overexpression traffic more and pause for less time (Fig $3.9 \mathrm{H})$. Together, these data show that over-expression of Kif1A rescues the decreased motility of S135E vesicles because of which S135E vesicles traffic similar to S135A vesicles.

\subsection{S135E vesicles are more localised at pre-synaptic sites}

After observing the decreased mobility of S135E vesicles, we next tested the localisation of Syt4 phosphomutants to axons versus dendrites and more specifically at pre- or postsynaptic sites. To distinguish axons and dendrites, we immunostained hippocampal cultures transfected with GFP-tagged Syt4, or S135A or S135E mutants with MAP2 to mark dendrites (Fig 3.10 A). We found no difference in localization of these vesicles in both dendrites and axons. In dendrites, the number of puncta per $10 \mu \mathrm{m}$ of control, S135A and S135E were $3.18 \pm 0.31 \%, 3.31 \pm 0.22 \%$ and $3.34 \pm 0.25 \%$, respectively (Fig 3.10 B). Similarly, in axons, the number of vesicles $/ 10 \mu \mathrm{m}$ was $2.66 \pm 0.14 \%, 2.42 \pm 0.14 \%$ and $2.85 \pm 0.17 \%$ for control, S135A and S135E, respectively (Fig 3.10 C).

Then, we specifically examined pre- and postsynaptic sites by co-transfecting Syt4 mutants with SypGFP or PSD-95 GFP, respectively (Fig 3.10 D and E). We found that S135E vesicles (that were least mobile in trafficking experiments) co-localized more with SypGFP compared to control (Pearson's coefficient of control, S135A and S135E were $0.37 \pm 0.024$, 
$0.38 \pm 0.030$ and $0.45 \pm 0.028$, respectively; $n=37,33$ and 33 cells from 3 different cultures)

(Fig 3.10 E).

A
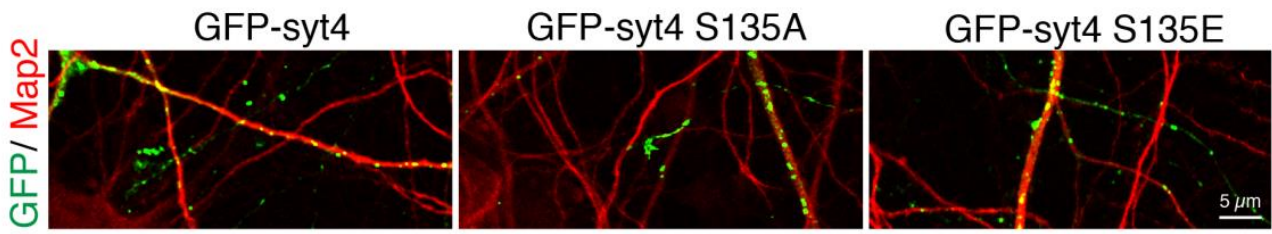

$\mathrm{B}$

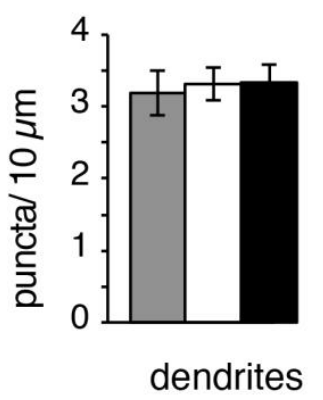

D

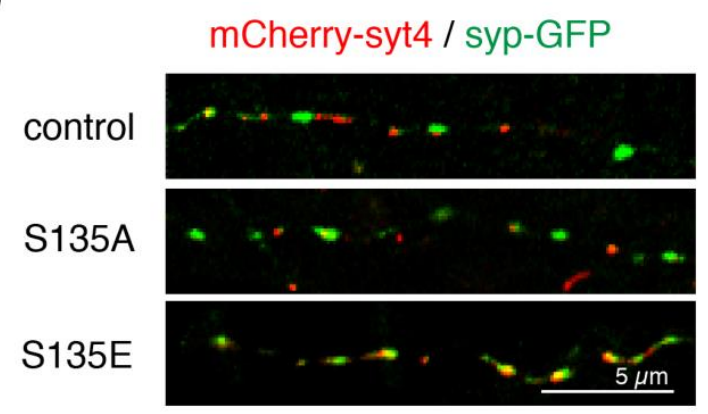

F

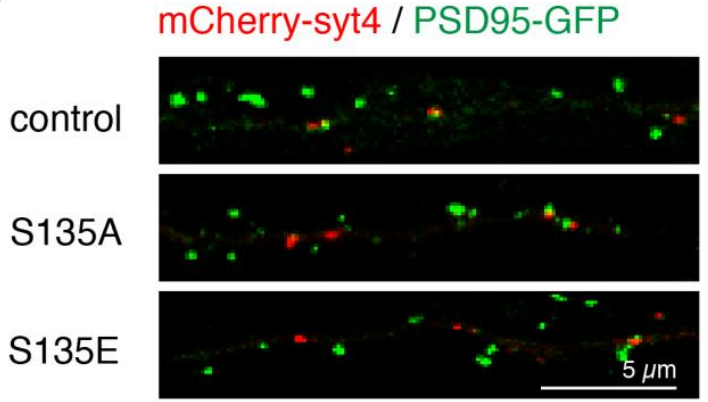

C

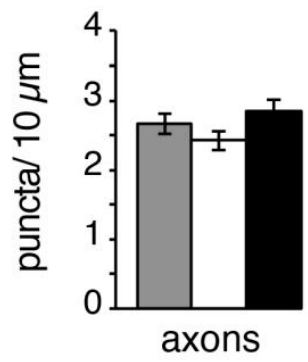

$E$

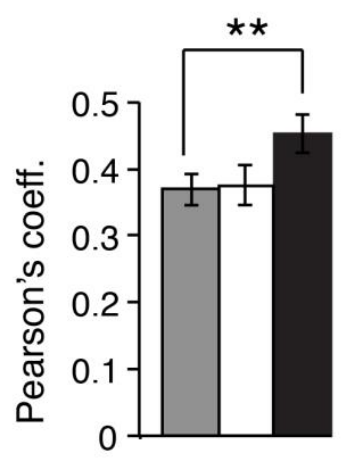

G

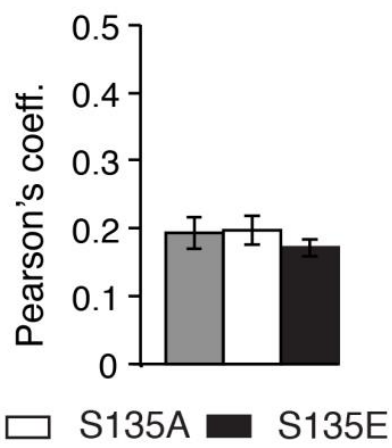

\section{Figure 3.10 Syt4 S135E vesicles are captured at pre-synaptic sites}

(A) Images of GFP-Syt4, GFP-Syt4 S135A and GFP-Syt4 S135E transfected cells immunostained for MAP2 to mark dendrites. (B) Quantitation of dendritic (GFP puncta in MAP2-positive processes) and axonal (C; GFP puncta in MAP-2 negative processes) vesicle distribution of GFP-Syt4, GFP-Syt4 S135A and GFP-Syt4 S135E. (D) Images of hippocampal neurons co-transfected with Syp-GFP and mCherry-tagged Syt4, Syt4 S135A or Syt4 S135E. (E) Quantified Pearson's coefficient showing that mCherry-Syt4 S135E is more co-localized at synapses than control or S135A Syt4 ( $n=37,33$ and 33 cells for Syp-GFP co-transfected with mCherry-Syt4, mCherry-Syt4 S135A and mCherry-Syt4 S135E, respectively, from 3 different cultures). (F) Images of neurons co-transfected with PSD95-GFP and mCherry-tagged Syt4, Syt4 S135A or Syt4 S135E. (G) Quantified Pearson's 
coefficient showing no difference in co-localization of PSD95-GFP with mCherry-Syt4, mCherry-Syt4 S135A or mCherry-Syt4 S135E ( $n=23,19$ and 20 cells for PSD95-GFP co-transfected with mCherry-Syt4, mCherry-Syt4 S135A and mCherry-Syt4 S135E, respectively, from 3 different cultures). Error indicates S.E.M. Significance was determined by Student's t-tests with Bonferroni correction (**p<0.01).

In contrast, colocalisation at post-synaptic sites marked with PSD-95GFP didn't show any significant difference (Pearson's coefficients were $0.19 \pm 0.02,0.20 \pm 0.02$ and $0.17 \pm 0.01$ for control, S135A and S135E, respectively) (Fig 3.10 F and G). These data show that phosphomimetic vesicles (S135E) are specifically captured at pre-synaptic sites.

\subsection{Disrupting actin increases the mobility of phosphorylated vesicles and does not change S135A vesicle trafficking}

In mature neurons, pre-synaptic sites are known to be enriched in F-actin that helps in modulating the efficacy of the terminal (Hirokawa et al., 1989; Cingolani and Goda, 2008). To verify this, we transfected hippocampal neurons at DIV10 with LifeAct-RFP and bassoonGFP. We then imaged at DIV14 and observed high co-localization of LifeAct-RFP with the pre-synaptic marker bassoon-GFP confirming concentrated F-actin at pre-synaptic sites (Fig

3.11 A). F-actin disruption has also been shown to increase the mobility of neuropeptidergic DCVs in PC-12 cells ( $\mathrm{Ng}$ et al., 2002). To test whether actin depolymerisation affects the mobility of DCVs in neurons, we treated our mCherry-tagged Syt4 control and phosphomutant transfected hippocampal neurons with $10 \mu \mathrm{M}$ of the actin depolymerizing agent latrunculin B for 30 minutes. We then imaged the treated and untreated control, S135A and S135E Syt4 transfected neurons. We observed a significantly higher average speed of latrunculin treated S135E and control vesicles compared to untreated (latrunculin treated control and S135 average speed was $0.18 \pm 0.0027 \mu \mathrm{m} / \mathrm{s}$ and $0.23 \pm 0.0044 \mu \mathrm{m} / \mathrm{s}$, respectively, whereas untreated control and S135 average speed were $0.15 \pm 0.0032 \mu \mathrm{m} / \mathrm{s}$ and $0.14 \pm 0.0025 \mu \mathrm{m} / \mathrm{s}$, respectively) (Fig 3.11 B). Vesicle displacement/s of control and S135E were also higher for treated cultures in comparison to untreated ones (displacement/s of control and S135E treated was $0.1 \pm 0.0025 \mu \mathrm{m} / \mathrm{s}$ and $0.12 \pm 0.0043 \mu \mathrm{m} / \mathrm{s}$, respectively, whereas for untreated control and S135E, it was $0.07 \pm$ $0.0031 \mu \mathrm{m} / \mathrm{s}$ and $0.06 \pm 0.0024 \mu \mathrm{m} / \mathrm{s}$, respectively) (Fig $3.11 \mathrm{C}$ ). Average speed and displacement/s for S135A vesicles in treated and untreated conditions were very similar (avg. speed of treated and untreated S135A vesicles were $0.18 \pm 0.0023 \mu \mathrm{m} / \mathrm{s}$ and $0.19 \pm$ $0.0027 \mu \mathrm{m} / \mathrm{s}$, respectively, whereas displacement/s of treated and untreated were $0.09 \pm$ 
$0.0022 \mu \mathrm{m} / \mathrm{s}$ and $0.09 \pm 0.0026 \mu \mathrm{m} / \mathrm{s}$ respectively). Interestingly, quantification of mobile vesicle percentage of treated and untreated conditions was not significantly different (Fig 3.11 D; $30.7 \pm 3.4 \%, 29.5 \pm 2.97 \%$ and $40.1 \pm 8.37 \%$ for treated control, S135E and S135A, respectively, and $24.7 \pm 3.36 \%, 33.9 \pm 3.88 \%$ and $23.1 \pm 2.57 \%$ for untreated control, S135E and S135A, respectively).

A

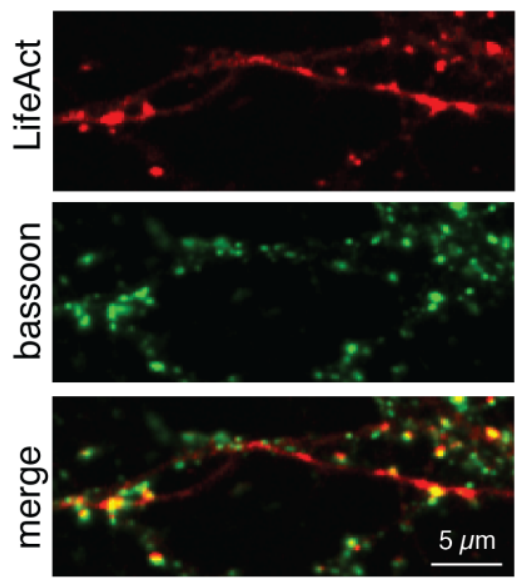

$\mathrm{D}$

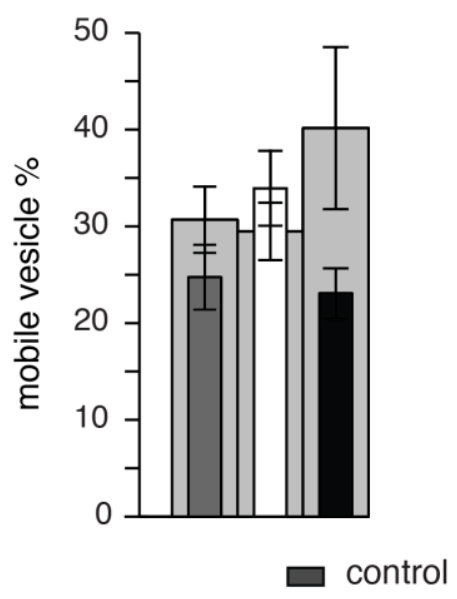

B

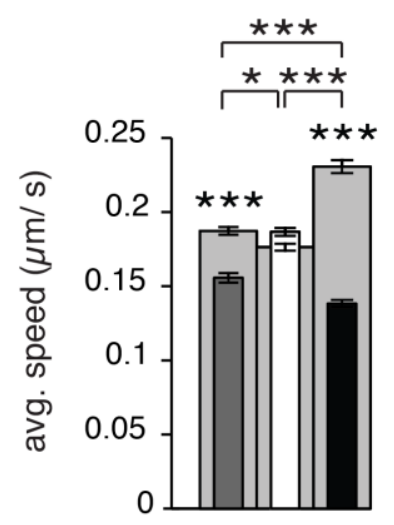

$\mathrm{E}$

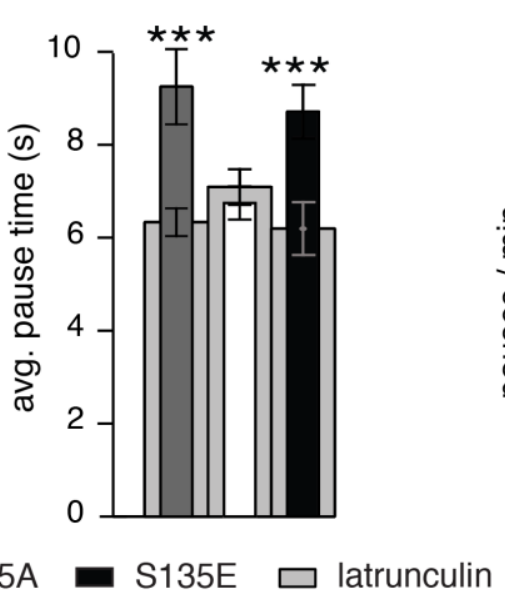

C

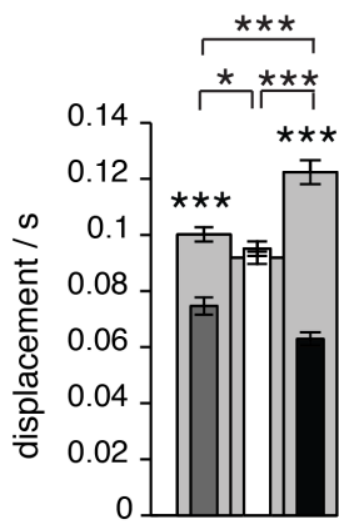

$\mathrm{F}$

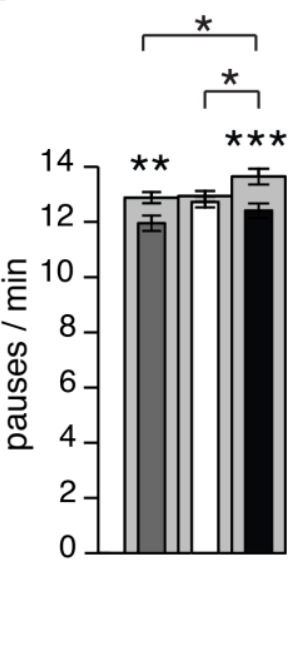

Figure 3.11 Actin disruption leads to more mobility of control and S135E vesicles without changing the trafficking parameters of S135A

(A) Example images of axons from hippocampal neurons transfected with LifeAct-RFP and Bassoon-GFP showing concentrated actin at pre-synaptic sites. (B) Quantification of average speed $(\mu \mathrm{m} / \mathrm{s})$ and displacement/s (C) of control, S135A and S135E in untreated and latrunculin treated conditions. Latrunculin treatment shows a significant increase in both average speed and displacement/s of control and S135E but not on S135A. (D) Percentage of mobile vesicles in latrunculin treated and non-treated conditions had no difference. (E) Average pause time of vesicles in latrunculin treated conditions was significantly lower than in non-treated conditions for control and S135E. (F) Quantification of pauses/min showed that latrunculin treated control and S135E vesicles had more pauses compared to non-treated conditions ( $n=5109,5609$ and 2317 for average speed, displacement/s, pauses/min and average pause time, whereas for mobile percentage $n=22,21$ and 15 videos from 4-5 different cultures for control, S135E and S135A, respectively in latrunculin 
treated conditions). Scale bar in fluorescent images is $5 \mu \mathrm{m}$. Error indicates S.E.M. Significance was determined by Student's t-tests with Bonferroni correction $\left({ }^{*} p<0.05,{ }^{* *} p<0.01\right.$ and $\left.{ }^{* * *} p<0.001\right)$.

We also analysed vesicle pauses for treated and untreated vesicles and observed that latrunculin treated S135E and control vesicles had more pauses per min but significantly less average pause time similar to highly mobile S135A untreated vesicles (Fig 3.11 E and F; $\mathrm{n}=5109,5609$ and 2317 vesicles for average speed, displacement/s, pauses/min and average pause time, whereas for mobile percentage $n=22,21$ and 15 videos from 4-5 different cultures for control, S135E and S135A, respectively, in latrunculin treated conditions). To summarize the above results, we found that actin plays a significant role in capturing phosphorylated DCVs at release sites.

\subsection{JNK phosphorylates Syt4 at the S135 site}

For capturing of vesicles, there is a need for unloading cargoes from microtubule tracks in axons. The c-Jun N-terminal kinase (JNK) pathway has been shown to involve in this step in cultured neurons (Stagi et al., 2006). In addition, JNK has been reported to phosphorylate Syt4 at S135 in PC-12 cells (Mori et al., 2008). To confirm that JNK and Syt4 interact, and JNK phosphorylates Syt4 we used biochemistry and in silico approaches. We co-transfected HEK 293 cells with Flag-MKK7-JNK1 and GFP-Syt4. In Co-IPs, we were able to pull down GFP-Syt4 with anti-Flag antibody and in reverse Co-IPs with an anti-GFP antibody we were able to pull down Flag-MKK7-JNK1 (Fig 3.12 A and B). We also used an in silico approach to determine if the S135 site of Syt4 is phosphorylated by JNK. We used c-Jun, a known substrate as a control for JNK kinase (Ip and Davis, 1998). Indeed, we found that JNK was the predicted kinase for phosphorylating Syt4 at S135 (Score/cut-off of JNK for Syt4 was 2.76 and of C-Jun was 3.84) (Fig 3.12 C).

In addition, we used an in vitro kinase assay to further confirm that Syt4 is phosphorylated by JNK at S135. We found that the percentage of JNK activity was reduced significantly (>50\%) when S135 in Syt4 WT was replaced with S135A (Fig 3.12 D). As a control, we used a c-Jun peptide containing S63, which is phosphorylated by JNK1. When the S63 site of cJun was replaced with S63A, we found an approximate two-third reduction in \% of JNK1 phosphorylation, similar to the reduction in the percentage of JNK activity for S135A Syt4. Co-IP experiments were performed with Dr. Saheeb Ahmed (a postdoc in the lab) and kinase prediction and in vitro kinase assays were done with our collaborator Mahdokht 
Kohansal, a PhD student in Prof. Reinhard Jahn's lab. These results cumulatively confirm that JNK phosphorylates Syt4 at the S135 site, which is consistent with Mori et al 2008.

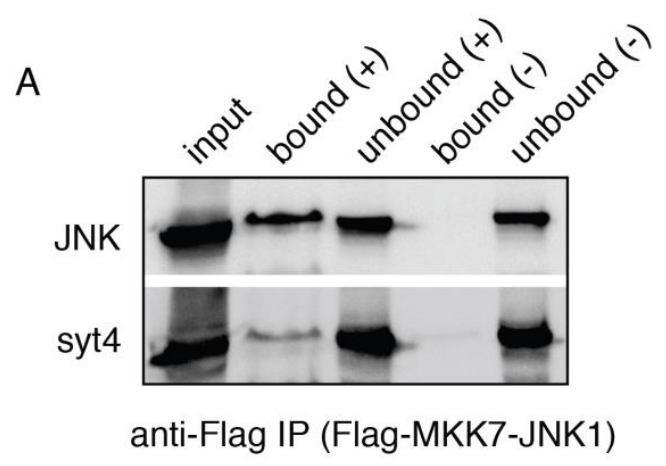

C

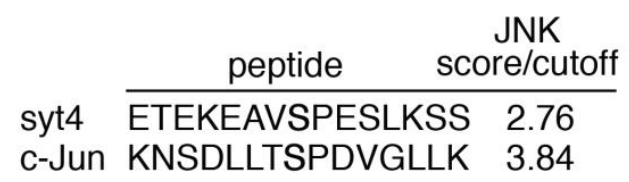

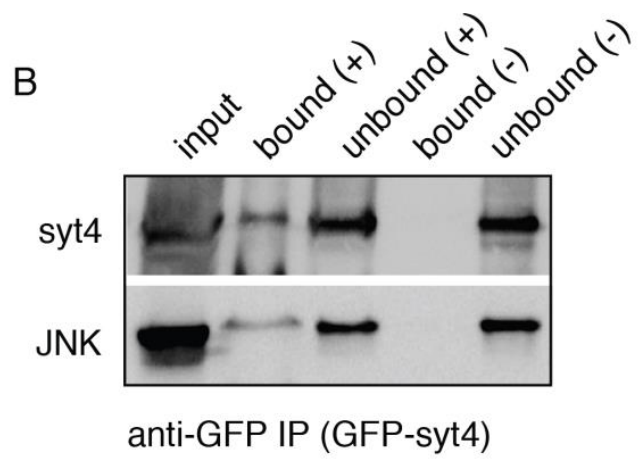

$\mathrm{D}$

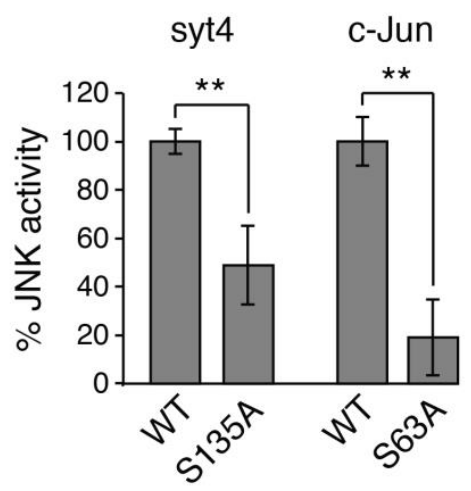

Figure 3.12 JNK phosphorylates Syt4 at the S135 site

(A) Western blots of JNK (Flag-MKK7-JNK1) and Syt4 (GFP-Syt4) co-immunoprecipitated from co-expressing HEK cells with anti-Flag and anti-GFP (B) antibodies. Plus sign indicates the pull-downs with anti-Flag or antiGFP antibodies attached to beads and minus signs show the binding controls. Respective bound and unbound fractions are indicated on the top of each lane. Antibodies used for Westerns are shown to the left of the blots. (C) GPS kinase prediction results showed that Syt4 and c-Jun (a known JNK substrate) peptide are phosphorylated by JNK (Score/cutoff of above 2 is a positive result). (D) In vitro kinase assay showed significantly higher percentage of JNK phosphorylation in Syt4 (WT) and c-Jun (WT) compared to phosphodeficient mutants. Error indicates S.E.M. Significance was determined by Student's t-tests with Bonferroni correction $(* * p<0.01)$.

\subsection{Phosphorylation of JNK at Syt4 S135 decreases the trafficking of DCVs}

To test the effect of JNK phosphorylation, specifically, on trafficking of Syt4 vesicles, we first tried activating and inhibiting JNK using pharmacological treatments with anisomycin (a JNK-activator) and SP600125 (a JNK inhibitor) (Bennett et al., 2001; Curtin and Cotter, 2002) in time-lapse imaging experiments of mCherry-Syt4 transfected hippocampal neurons. We saw a significant decrease in the mobility of Syt4 vesicles with anisomycin treatment, but little or no change in vesicle trafficking upon SP600125 treatment. Analysed tracks in Imaris and pause detection by kymographs showed that Syt4 vesicles were highly 
mobile in control and SP600125 treated cultures, whereas anisomycin treatment decreased the mobility of these vesicles (Fig 3.13 A and B).

Frequency distributions also showed that anisomycin treated cultures had more vesicles with low average speed (Fig 3.13 C). We found that mCherry-Syt4 vesicles in cultures treated with anisomycin had a significant decrease in average speed of vesicles and displacement/s compared to control and SP600125 treated cells (Fig 3.13 D and E). Frequency distribution and average speed quantification showed that anisomycin treated Syt4 vesicles had the lowest average speed of $0.105 \pm 0.0024 \mu \mathrm{m} / \mathrm{s}$ compared to control and SP600125 treated cultures (control avg. speed was $0.145 \pm 0.0030 \mu \mathrm{m} / \mathrm{s}$ and SP600125 treated culture average vesicle speed was $0.147 \pm 0.0028 \mu \mathrm{m} / \mathrm{s})$. Vesicle displacement $/ \mathrm{s}$ also showed that SP600125 treated and control Syt4 vesicles covered more distance compared to anisomycin treated vesicles (displacement/s of control, SP600125 and anisomycin treated vesicles was $0.074 \pm 0.0068 \mu \mathrm{m} / \mathrm{s}, 0.063 \pm 0.007 \mu \mathrm{m} / \mathrm{s}$ and $0.047 \pm$ $0.0075 \mu \mathrm{m} / \mathrm{s}$, respectively; $\mathrm{n}=2643,2362$ and 2151 vesicles for average speed, displacement/s, pauses/min and avg. pause time of control, SP6 and anisomycin treated cultures, respectively). We also examined mobile vesicle percentage and found that anisomycin treated cultures had a trend towards less mobile vesicle (Fig 3.13 F). The percentage of mobile vesicles in anisomycin treated cultures was $19.7 \pm 3.01 \%$ which was significantly less compared to mobile vesicle percentage of control and SP600125 treated cultures $(29.7 \pm 3.06 \%$ and $25.3 \pm 1.91 \%$, respectively; $n=12,9$ and 16 videos for control, SP6 and anisomycin, respectively from 3 different cultures).

In addition, we found by pause analysis of these treated cultures that there were significantly less pauses in anisomycin treated cells and higher average pause time compared to control and SP600125 treated cultures (Fig 3.13 G and H). Trafficking parameters of SP600125 treated cells were not significantly different compared to control. In addition, Westerns also showed that anisomycin treated cultures had significantly higher p-JNK compared to control and SP600125 treated cultures (Fig 3.13 I; Westerns were done by Dr. Saheeb Ahmed). We found that p-JNK protein levels in control and SP600125 treated cultures were not significantly different, which explains why we did not see a change in trafficking of vesicles with SP6 treatment of hippocampal neurons. These results show that 
upon anisomycin treatment (which activates JNK), the mobility of Syt4 DCVs decreases substantially compared to control and SP600125 treated cultures.

A
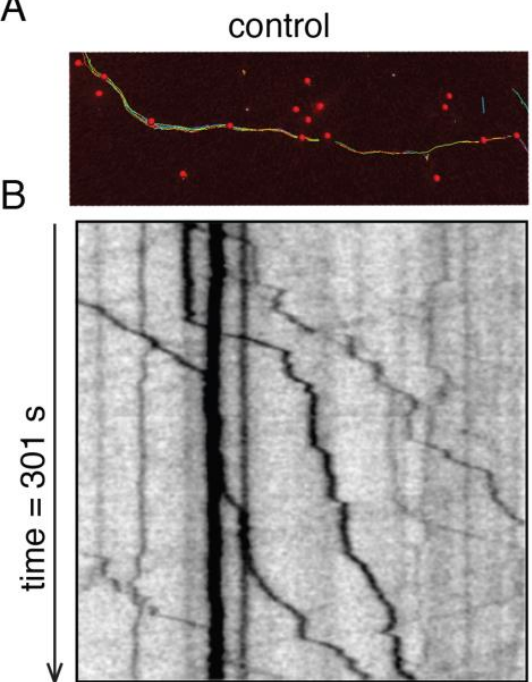

C

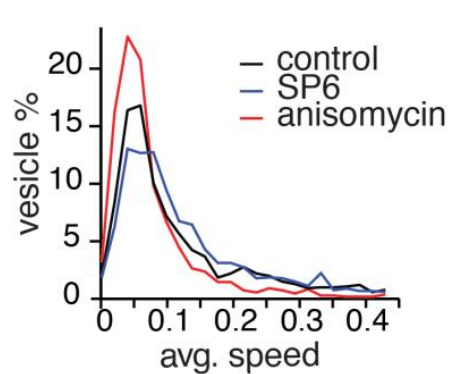

G

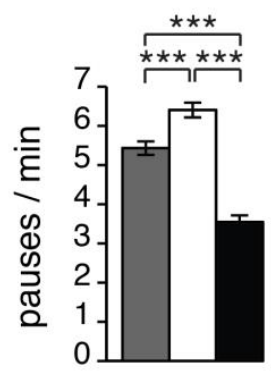

D

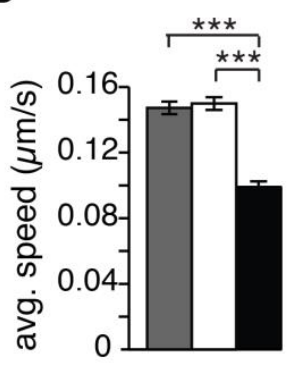

$\mathrm{H}$

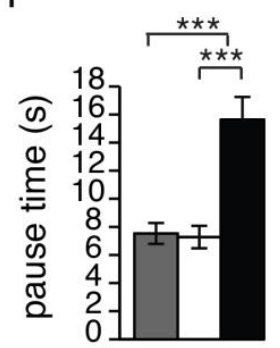

SP600125
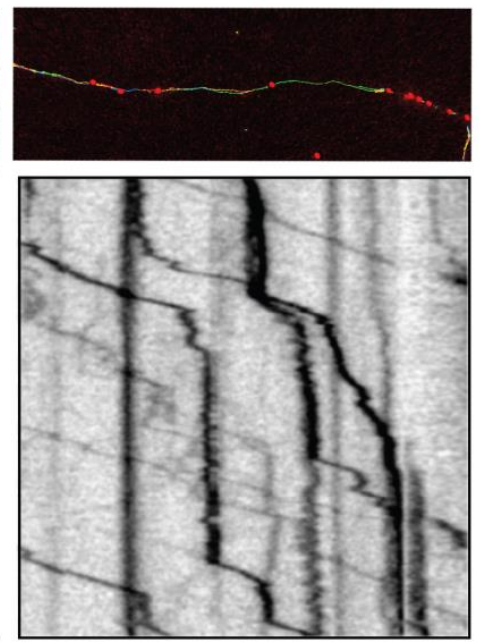

E
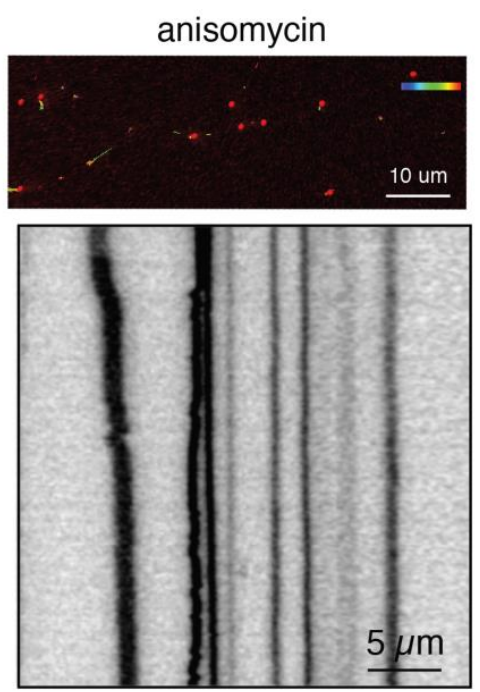

$\mathrm{F}$
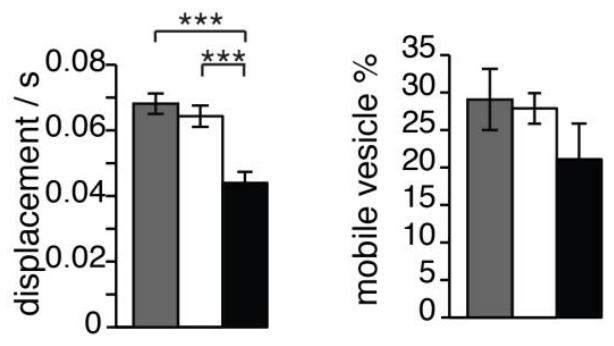

I
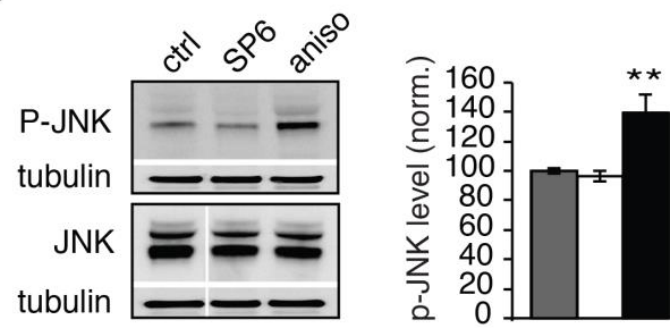

$\square$ Control $\square$ SP600125

anisomycin

Figure 3.13 Anisomycin and SP600125 effect on trafficking of DCVs

(A) Snapshots of trafficking videos of control, SP600125 and anisomycin treated hippocampal neurons. Scale bar is $10 \mathrm{um}$. (B) Respective kymographs of all conditions. Scale bar is $5 \mu \mathrm{m}$. (C) Comparison of frequency distribution of avg. speed of control, SP600125 and anisomycin conditions. (D) Quantified average speed $(\mu \mathrm{m} / \mathrm{s})$ and displacement $/ \mathrm{s}(\mathrm{E})$ shows that vesicles in the anisomycin treated condition have the lowest speed and cover the least distance. (F) Mobile vesicle percentage was quantified. (G) Pauses/ min and average pause time (H) was quantified for control, SP600125 and anisomycin treated conditions ( $n=2643,2362$ and 2151 vesicles for average speed, displacement/s, pauses/min and avg. pause time of control, SP600125 and anisomycin treated conditions, respectively whereas for mobile percentage $n=12,9$ and 16 videos for control, SP600125 and anisomycin treated conditions, respectively, from 3 different cultures). (I) Western for JNK and p-JNK in hippocampal neuron cell lysates of SP600125 and anisomycin treated samples compared 
to control. Right panel indicates quantification of $\mathrm{p}-\mathrm{JNK}$ band intensity in all three conditions. Error indicates S.E.M. Significance was determined by Student's t-tests with Bonferroni correction $\left({ }^{*} p<0.05,{ }^{* *} p<0.01\right.$ and $* * * p<0.001)$.

\subsection{Phosphorylation of Syt4 S135 by over-expressing JNK also reduces the trafficking of DCVs}

Considering the unspecific effects pharmacological treatments could have, we tested the effect of JNK phosphorylation of Syt4 vesicles using a more specific approach. We transfected hippocampal neurons with control mCherry-Syt4, mCherry-Syt4-P2A-JNK1 (APF), a dominant negative form of JNK1, or mCherry-Syt4-P2A-MKK7-JNK1 to examine the effects of increased activated JNK1 on Syt4 trafficking in isolated cells (Derijard et al., 1994; Lei et al., 2002; Kim et al., 2011). To further confirm that the S135 site of Syt4 is phosphorylated by JNK, we transfected hippocampal neurons with mCherry-Syt4 (S135A)P2A-MKK7-JNK1. We examined vesicle trafficking by time-lapse imaging in these four conditions at DIV11, and analysed vesicle mobility. We observed high mobility of vesicles in JNK1 (APF) and S135A/MKK7-JNK1 whereas control Syt4 vesicles with over-expressed MKK7-JNK1 were least mobile both by Imaris and kymograph analysis (Fig 3.14 A). Frequency distributions of average speed $(\mu \mathrm{m} / \mathrm{s})$ also showed more vesicles with MKK7JNK1 co-expression with slower speed whereas S135A vesicles with MKK7-JNK1 coexpression had higher speeds (Fig 3.14 B). Average speed and displacement/s of vesicles in JNK1 (APF) and S135A/MKK7-JNK1 conditions were significantly higher than control and MKK7-JNK1 conditions (Fig 3.14 C and D). Mobile vesicle percentage of Syt4 MKK7-JNK1 was the lowest compared to control, JNK1 (APF) and S135A/MKK7-JNK1 conditions (Fig 3.14 E). Vesicle pause analysis revealed that vesicles that were highly mobile, as in JNK1 (APF) and S135A/MKK7-JNK1 conditions, had a higher number of pauses/min but significantly less average pause time(s). On the other hand, pauses/min of control and MKK7-JNK1 were significantly lower whereas average pause time in these conditions was significantly higher than JNK1 (APF) or S135A/MKK7-JNK1 conditions (Fig 3.14 F and G).

Furthermore, we tested the co-localization of control, JNK1 (APF) and MKK7-JNK1 vesicles with the pre-synaptic marker Syp-GFP by ICC (Fig 3.14 H). We found that MKK7-JNK1 vesicles were highly co-localised with Syp-GFP compared to other conditions indicating that these least mobile vesicles are more localised at pre-synaptic sites in hippocampal neurons 
(Fig 3.14 I; Pearson's coefficient was $0.38 \pm 0.04$ for MKK7-JNK1, $0.33 \pm 0.02$ for JNK1 (APF) and $0.30 \pm 0.02$ for control).

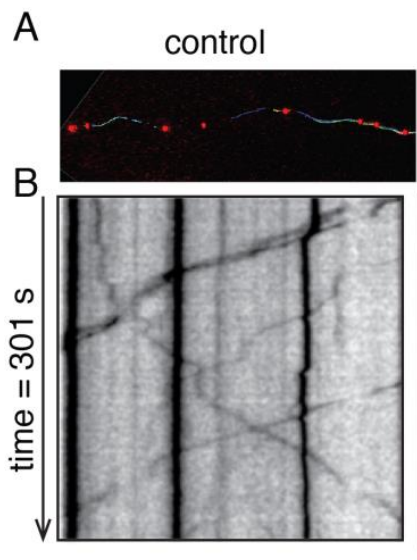

C

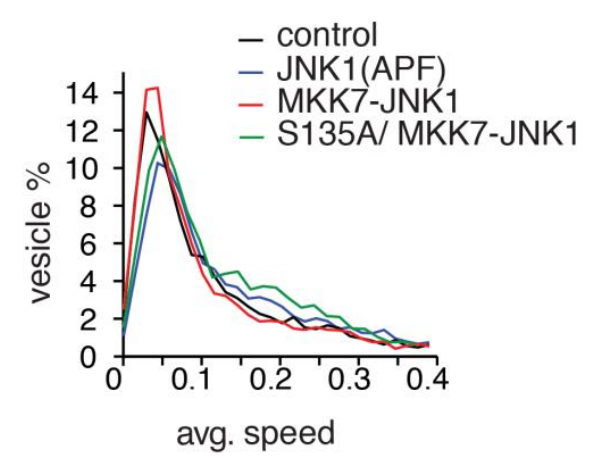

$\mathrm{F}$

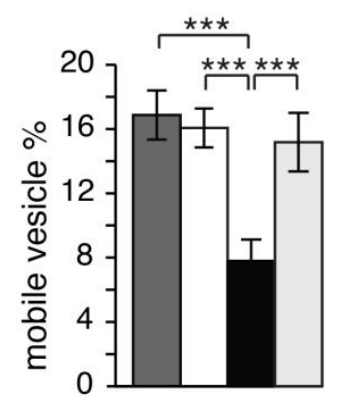

I
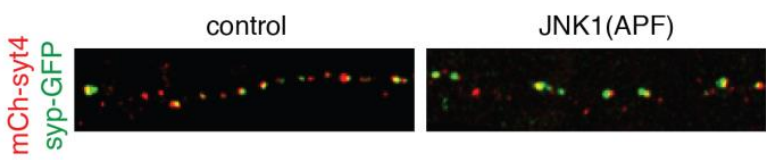

D

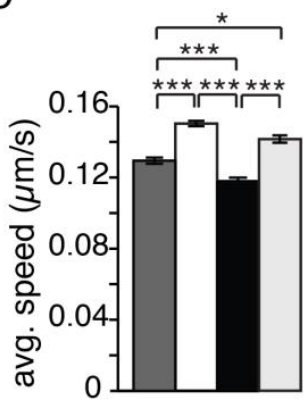

G

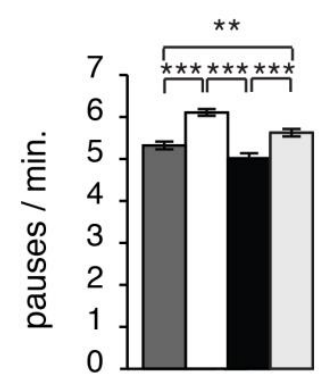

S135A/ MKK7-JNK1
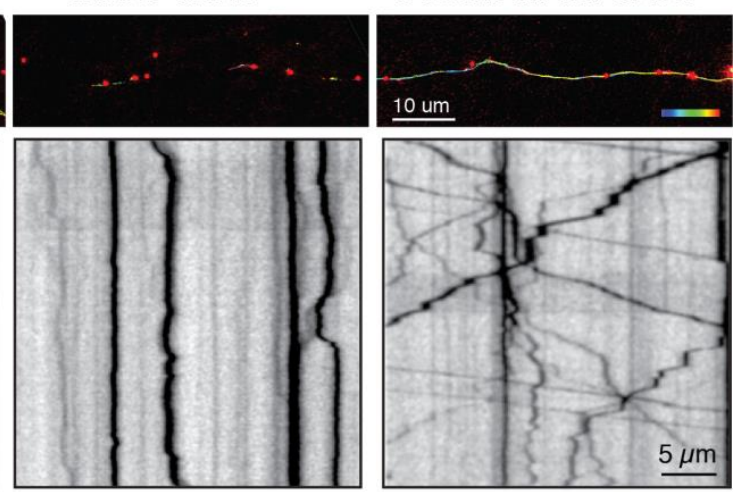

E

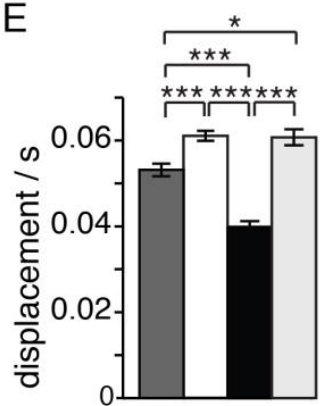

$\mathrm{H}$

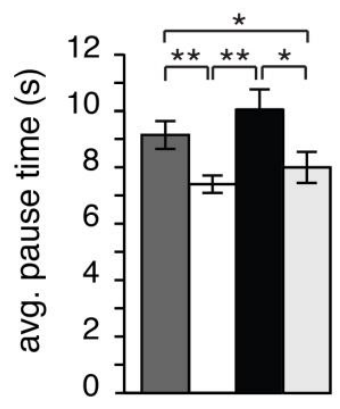

$\square$ control $\square$ JNK1(APF) $\square$ MKK7-JNK1 $\square$ S135A/MKK7-JNK1

Figure 3.14 Phosphorylation of Syt4 at S135 by JNK decreases DCVs mobility and localises these vesicles to synapses

(A) Images from videos of hippocampal neurons transfected with Syt4 control, Syt4 control with dominant negative JNK (JNK1(APF)), Syt4 control with over-expressed JNK (MKK7-JNK1) and Syt4 S135A with overexpressed JNK (S135A/MKK7-JNK1). Scale bar is $10 \mu \mathrm{m}$. (B) Respective kymographs of all conditions. Scale bar 
is $5 \mu \mathrm{m}$. (C) Frequency distribution of average speed of vesicles in control, JNK1 (APF), MKK7-JNK1 and S135A/MKK7-JNK1 conditions. (D) Quantified avg. speed and displacement/s (E) showed that JNK1(APF) and S135A/MKK7-JNK1 vesicles moved with significantly higher speed and covered larger distances compared to control and MKK7-JNK1 conditions. (F) Mobile vesicle percentage quantification indicates that vesicles in the MKK7-JNK1 condition were the least mobile of all conditions. (G) Quantification of pauses/min shows that JNK1(APF) and S135A/MKK7-JNK1 have a higher number of pauses/min compared to control and MKK7-JNK1 conditions. (H) Average pause time of vesicles in the MKK7-JNK1 condition was the highest compared to other conditions ( $n=5216,7478,3172$ and 4232 vesicles for average speed, displacement/s, pauses/min and avg. pause time of control, JNK1(APF), MKK7-JNK1 and S135a/MKK7-JNK1, respectively, whereas for mobile percentage $n=22,27,27$ and 18 videos from 4 different cultures). (I) Images of neurons co-transfected with Syp-GFP and control, JNK1(APF) or MKK7-JNK1. Scale bar is $10 \mu \mathrm{m}$. (J) Quantified Pearson's coefficient showing more colocalisation of vesicles with Syp-GFP in the MKK7-JNK1 condition compared to all other conditions ( $n=12,17$, and 21 cells for control, JNK1(APF) and MKK7-JNK1 conditions, respectively, from 3 different cultures). Error indicates S.E.M. Significance was determined by Student's t-tests with Bonferroni correction $\left({ }^{*} p<0.05,{ }^{* *} p<0.01\right.$ and $\left.{ }^{* * *} p<0.001\right)$.

These data reveal that JNK phosphorylation of Syt4 specifically at S135 leads to a decrease in the mobility of transiting Syt4 vesicles and increases their localisation at synapses in hippocampal neurons.

\subsection{Activity-dependent phosphorylation of S135 captures transiting Syt4 vesicles}

At the Drosophila NMJ, the probability of capturing transiting vesicles depends on neuronal activity. Highly active synapses capture vesicles from the transiting pool, which maintains the neuropeptidergic store at synapses (Shakiryanova et al., 2006; Bulgari et al., 2014). To determine whether the same phenomenon is true for Syt4-harboring DCVs in mammalian hippocampal neurons, we examined the trafficking of Syt4 vesicles by transiently increasing neuronal activity. First we transfected hippocampal cultures at DIV10 with mCherry-Syt4 control, mCherry-Syt4 S135A or mCherry-Syt4-P2A-JNK1 (APF). We applied high KCl solution for 3 minutes to depolarize neurons in the middle of 10-minute time-lapse recordings at DIV11. In mCherry-Syt4 WT, we found that many transiting vesicles were stopped during the time of $\mathrm{KCl}$ stimulation (Fig 3.15 A). These vesicles then either resumed movement at the cessation of stimulus, or remained immobilized. Whereas in the case of mCherry-Syt4 S135A, there were very few pausing vesicles during the stimulation time (Fig $3.15 \mathrm{~B})$. 
A

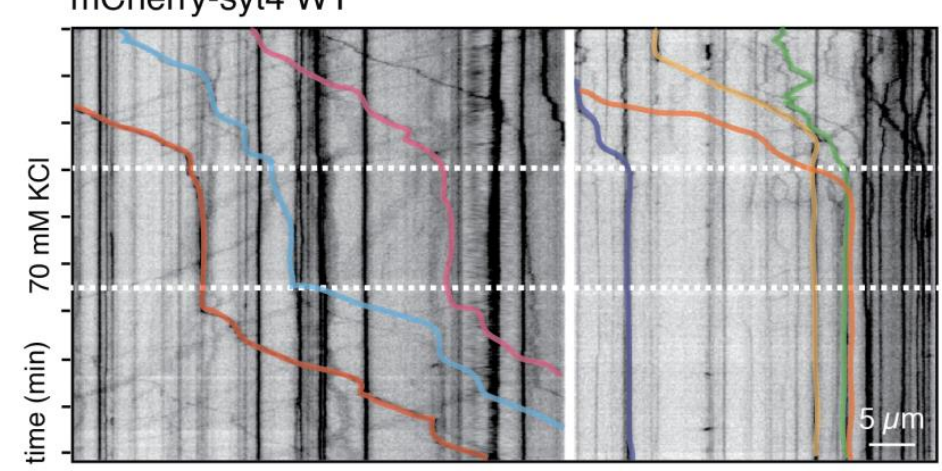

B mCherry-syt4 S135A

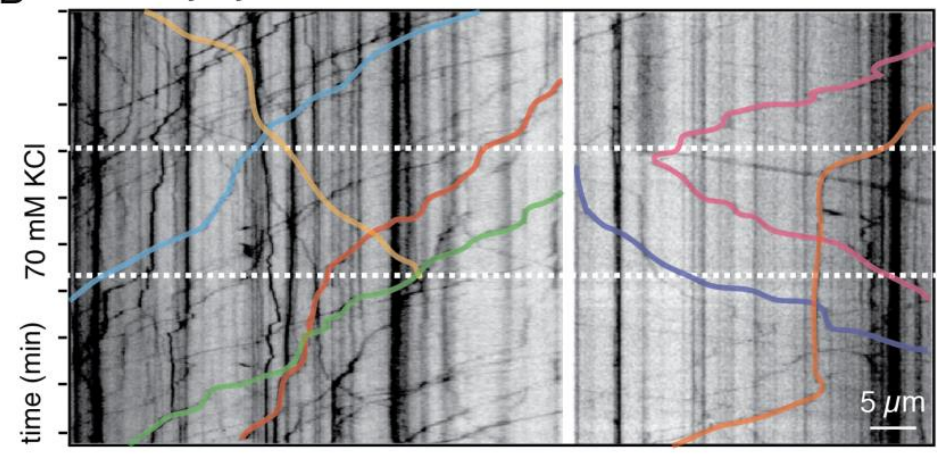

C mCherry-syt4 / JNK1(APF)

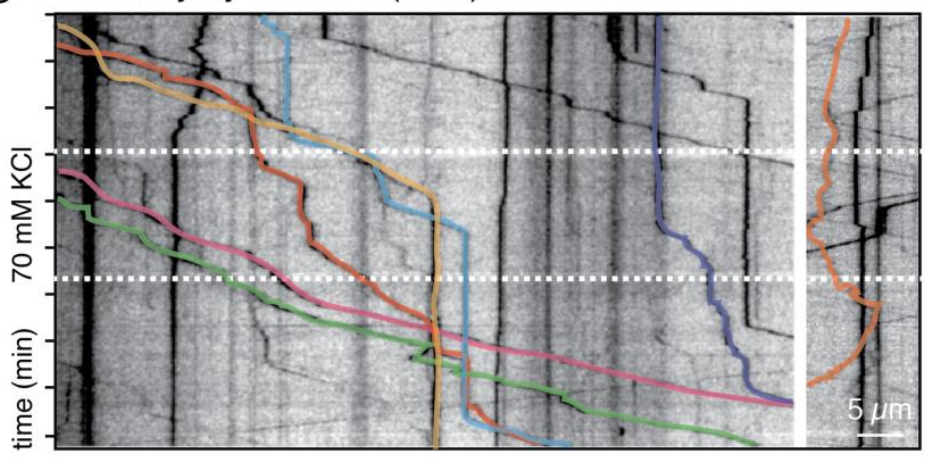

D mCherry-syt4 WT

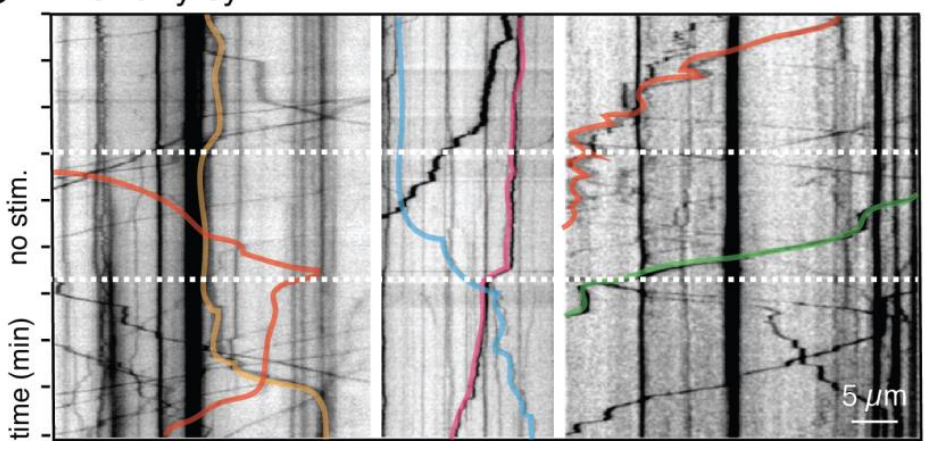

E

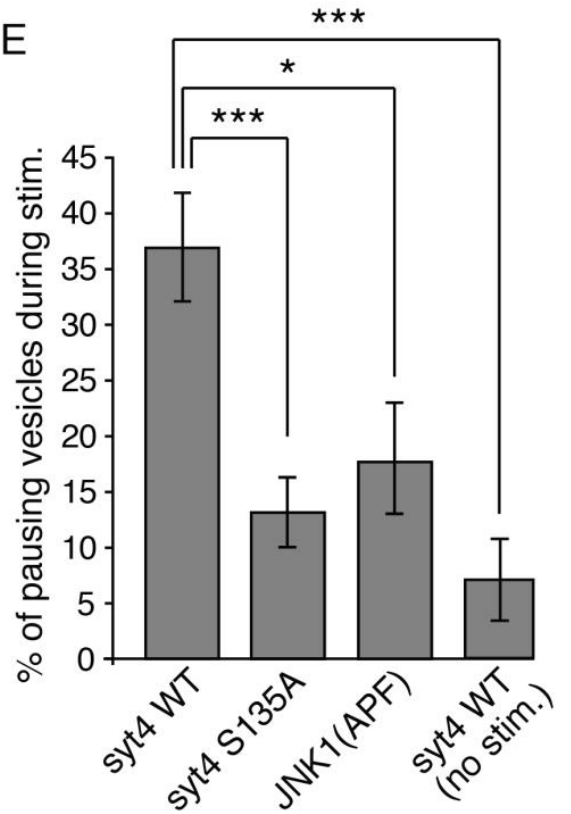

Figure 3.15 Activity dependent phosphorylation causes transiting vesicles to pause.

(A) Kymographs of Syt4 vesicle trafficking in mCherry-Syt4 WT, mCherry-Syt4 S135A (B) and mCherry-Syt4 WT/JNK1 (APF) (C) conditions during stimulation with $70 \mathrm{mM} \mathrm{KCl}$. Dotted white line represents the $70 \mathrm{mM}$ $\mathrm{KCl}$ application during time-lapse imaging. (D) Kymograph of mCherry-Syt4 WT vesicles without $\mathrm{KCl}$ stimulation. Coloured tracks highlight the trajectories of moving vesicles. In mCherry-Syt4 WT conditions, there were more pausing vesicles during $\mathrm{KCl}$ stimulation compared to mCherry-Syt4 S135A and mCherry-Syt4 WT/ JNK1 (APF) conditions. (E) Quantification of percentage of vesicles pausing during stimulation (and 
without stimulation for Syt4 WT) shows that Syt4 WT has significantly more pauses compared to Syt4 S135A and Syt4/ JNK1 (APF) during stimulation. Scale bar is $5 \mu \mathrm{m}(n=17,15$ and 9 videos for stimulated control, S135A and JNK1 (APF) conditions, respectively, from 3-4 different cultures and $n=8$ videos for non-stimulated Syt4 control from 3 different cultures). Error indicates S.E.M. Significance was determined by Student's t-tests with Bonferroni correction ( ${ }^{*} p<0.05$ and $\left.{ }^{* * *} p<0.001\right)$.

We further examined mCherry-Syt4-P2A-JNK1 (APF) to test if JNK phosphorylation of Syt4 is necessary for activity dependent capture, and found that Syt4 vesicles co-expressed with dominant negative JNK1 (APF) remained highly mobile during neuronal activity. This confirmed that JNK1 phosphorylation of Syt4 S135 was necessary for activity-dependent pausing of transiting DCVs in hippocampal neurons (Fig. 3.15 C).

As a control, we also imaged mCherry-Syt4 without $\mathrm{KCl}$ stimulation and found low numbers of pausing vesicles comparable to mCherry-Syt4 S135A or mCherry-Syt4-P2A-JNK1 (APF) conditions.

There was a significantly higher percentage of pausing vesicles in Syt4 (WT) compared to Syt4 S135A and Syt4/JNK1 (APF) conditions (Fig 3.15 E; percentage of pausing vesicles in three conditions were: $36.8 \pm 4.9 \%$ (Syt4 (WT)), $13.1 \pm 3.08 \%$ (Syt4 S135A) and $18.1 \pm 5.02 \%$ (Syt4/JNK1 (APF)); $n=17,15$ and 9 videos of WT, S135A and JNK1 (APF), respectively from 3-4 different cultures in $\mathrm{KCl}$ stimulation conditions and $\mathrm{n}=8$ videos for non-stimulated Syt4 WT from 3 different cultures). Together, these data show that activity dependent JNK phosphorylation captures transiting neuropeptidergic dense core vesicles in hippocampal neurons. 


\subsection{PJNK is localised to synapses at DIV13 in hippocampal neurons}

We then aimed to visualize the localisation of JNK and p-JNK in hippocampal neurons. We used JNK and p-JNK antibodies in combination with Syp, a pre-synaptic site marker and Map2, a dendritic marker, to mark endogenous localisation of JNK and p-JNK. We found that JNK was present throughout hippocampal neurons, in cell body, neurites, and synapses, whereas p-JNK was more localised to synapses (Fig 3.16 A). Next, we compared p-JNK levels at DIV6 and DIV13. We immunostained neurons with p-JNK, the pre-synaptic marker Syp, and the dendritic marker Map2 at DIV6 and 13. We found significantly more p-JNK localised to synapses at DIV13 compared to DIV6 (Fig 3.16 B). We checked the total protein levels by Western blotting for JNK and p-JNK at DIV6 and DIV13 and found that the total protein levels were quite similar, although with a bit of variation among cultures (Fig $3.16 \mathrm{C})$.

A
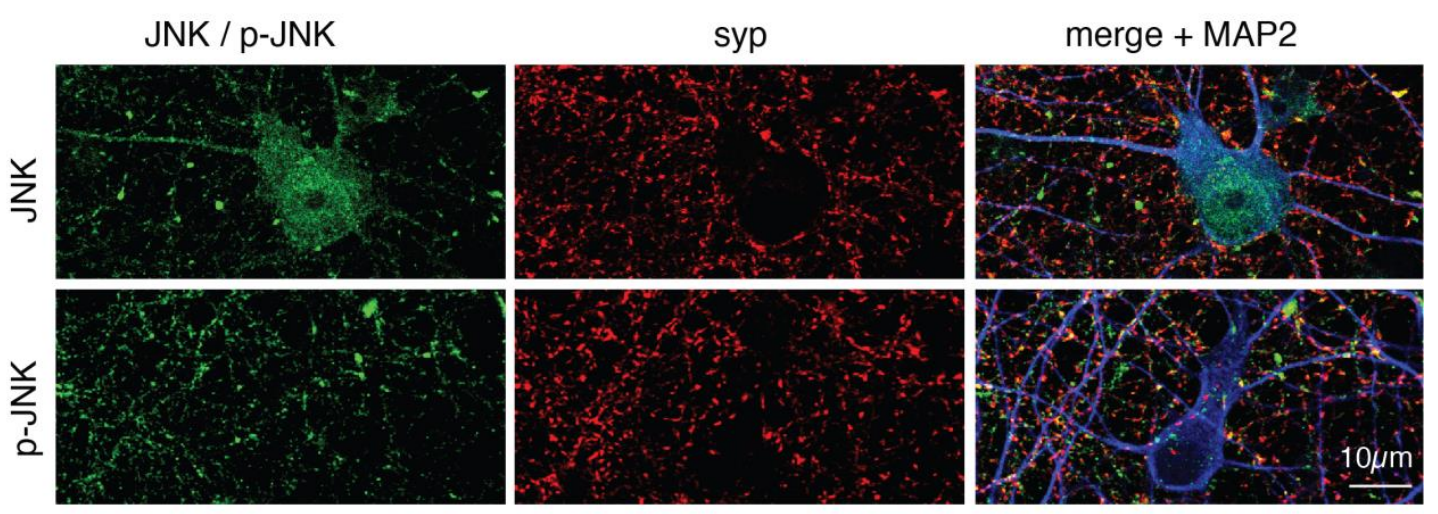

B
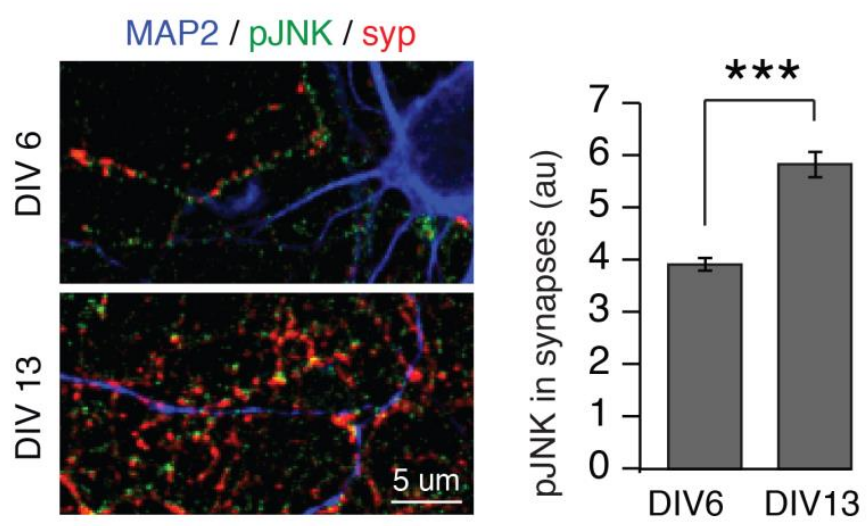

C

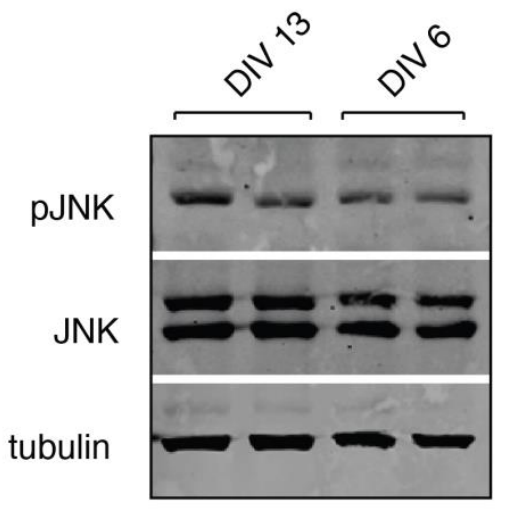

Figure 3.16 Localisation of JNK and p-JNK in hippocampal neurons.

(A) Example images to show the localisation of JNK and p-JNK with syp (a synapse marker). MAP2 is a dendritic marker. Scale bar is $10 \mu \mathrm{m}$. (B) Comparison and quantification of $p$-JNK (green) levels at synapses marked with Syp (red) at DIV6 and 13. Scale bar is $5 \mu \mathrm{m}$ ( $n=27$ images each for DIV6 and 13). (C) Western blot comparing total protein levels of JNK and p-JNK at DIV6 and 13 ( $n=2$ different neuronal cultures). Error indicates S.E.M. Significance was determined by Student's t-tests with Bonferroni correction $(* * * p<0.001)$. 


\subsection{Activity dependent capture of Syt4 vesicles at synapses by JNK phosphorylation}

Knowing that p-JNK is concentrated more at synapses (Fig 3.16 A) and that short term $70 \mathrm{mM} \mathrm{KCl}$ depolarisation causes transient capture of mCherry-Syt4 vesicles (Fig. 3.15 A), we tested the effects of stimulating neuronal cultures for longer times by treating them with $40 \mu \mathrm{M}$ bicuculline for $1 \mathrm{~h}$. We then performed immunostaining experiments to examine if neuronal stimulation leads to capture of endogenous Syt4 at synapses by JNK phosphorylation, by testing if adding the JNK inhibitor SP600125 reduces capture in neuronal cultures in which activity is increased.

To prove that SP600125 blocks JNK activity, we treated DIV13 hippocampal neuronal cultures with anisomycin (a JNK activator), SP600125 (a JNK inhibitor) and with both SP600125 and anisomycin and then blotted for P-c Jun, a known substrate for JNK (Bogoyevitch and Kobe, 2006). We found that SP600125 indeed inhibited JNK phosphorylation, as treatment of hippocampal neurons with anisomycin (JNK activator) increased P-c Jun levels whereas addition of SP600125 with anisomycin blocked the increase in P-c Jun (Fig 3.17 A). Bicuculline treatment increased p-JNK levels slightly and also increased Syt4, as expected, since Syt4 is upregulated by activity (Fig $\mathbf{3 . 1 7}$ B; Dr. Saheeb Ahmed, a postdoc in the lab performed these Westerns).

We then performed immunostaining experiments in hippocampal neurons in control, bicuculline treated ( $40 \mu \mathrm{M}$ bicuculline for $1 \mathrm{~h}$ ) and SP600125 with bicuculline treated conditions. We immunostained these cultures with antibodies for endogenous p-JNK or Syt4 and Syp as a synapse marker (Fig 3.17 C and E). We observed a significant increase in the level of p-JNK at synapses in bicuculline treated cultures, whereas this increase was reduced to basal levels with concomitant SP600125 treatment (Fig 3.17 D). Similarly, we found a significant increase in Syt4 at synapses with bicuculline treatment, which was reduced to basal levels with SP600125 treatment (Fig 3.17 F). With these experiments we showed that increased neuronal activity enhances p-JNK at synapses, which leads to capture of Syt4 vesicles at synaptic sites in hippocampal neurons. 

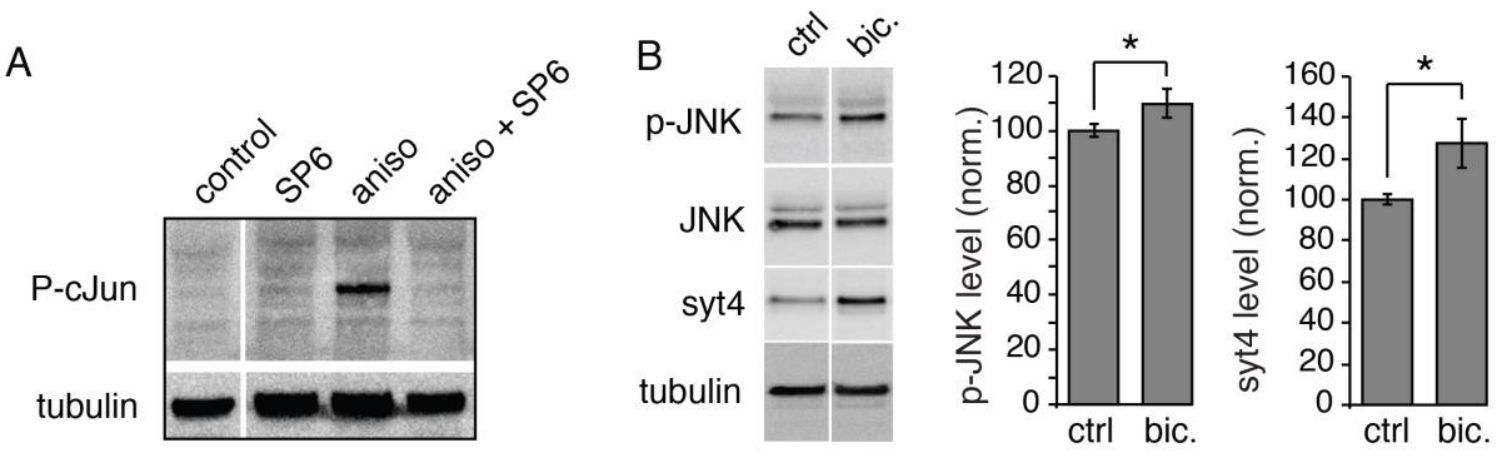

C
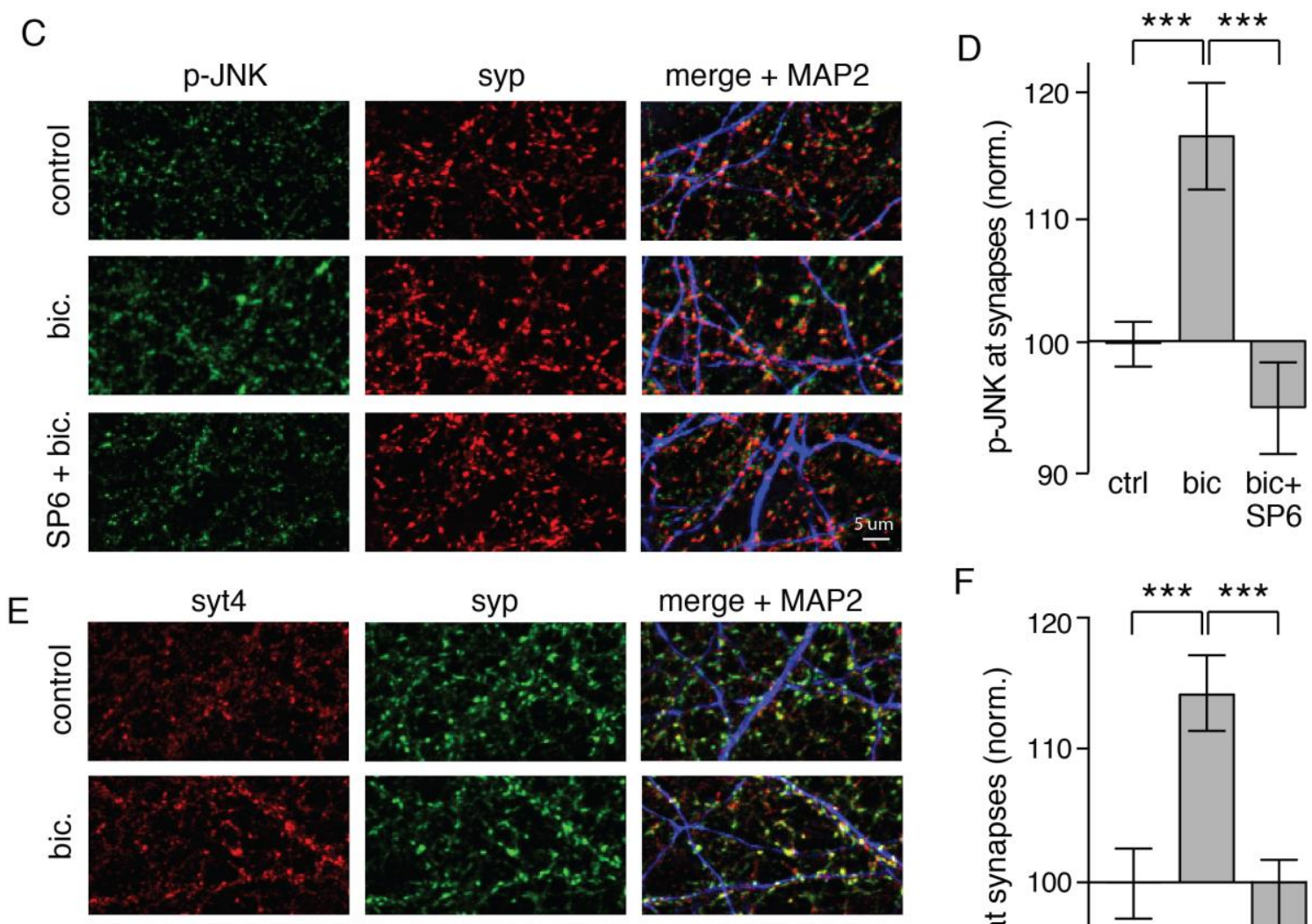

$\mathrm{F}$

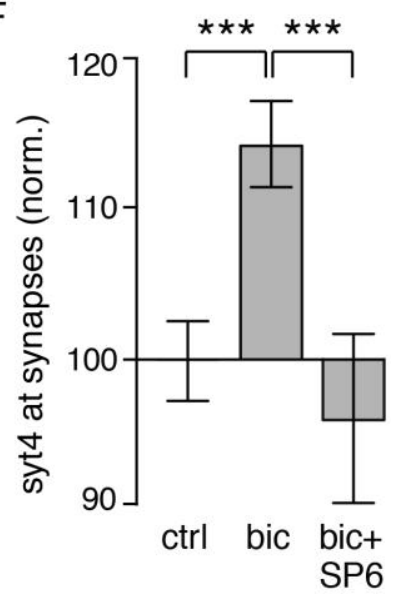

Figure 3.17 Long term stimulation causes more p-JNK and Syt4 at synapses.

(A) Western blot of P-c Jun in hippocampal neurons treated with SP600125 (a JNK inhibitor), anisomycin and anisomycin + SP600125 compared to control. (B) Westerns for Syt4, JNK and p-JNK in hippocampal neurons treated with Bicuculline (bic) for $1 \mathrm{hr}$ compared to control. Quantification of $\mathrm{p}$-JNK and Syt4 levels in Westerns comparing control (ctrl) and bicuculline treated (bic) cultures. (C) Example images of p-JNK (red), syp (green) and merged images with Map2 (a dendrite marker) in control, bicuculline treated and bicuculline + SP600125 treated conditions. (D) Quantification of $p$-JNK at synapses in all three conditions. (E) Example images of Syt4 (red), syp (green) and merged images with Map2 (a dendrite marker) in control, bicuculline treated and bicuculline + SP600125 treated cultures. (F) Quantification of p-JNK at synapses in all three conditions ( $n=46$, 49 and 17 images in control, bicuculline treated and bicuculline + SP600125 treated conditions, respectively, from 4 different cultures). Scale bar $5 \mu \mathrm{m}$. Error indicates S.E.M. Values normalised to control in (D) and (F). Significance was determined by Student's t-tests with Bonferroni correction $\left({ }^{*} p<0.05\right.$ and $\left.{ }^{* * *} p<0.001\right)$. 


\subsection{Phosphorylation of Syt4 at S135 doesn't change the fusion of DCVs at synaptic or non-synaptic sites in hippocampal neurons}

After determining the effects of phosphorylation of Syt4 at S135 on trafficking and capture of DCVs, we extended our study to examine if phosphorylation of Syt4 also changes fusion properties of DCVs in axons. We co-transfected hippocampal neurons with synapsinmCherry (to mark synaptic sites) and pHluorin-tagged constructs of control Syt4, Syt4 S135A and Syt4 S135E at DIV10. PHluorins are pH sensitive GFPs, which are normally fused to the lumenal domain of vesicle proteins and quenched in acidic environments, i.e. in the lumen of vesicles, but become fluorescent in basic environments, i.e. when vesicles fuse with the plasma membrane and their lumenal domain is exposed to the extracellular solution (Miesenbock et al., 1998). We used constructs in which the pHluorin molecule was attached to the lumenal domain of Syt4 and Syt4 phosphomutants to test for differences in fusion properties. We stimulated transfected cells at DIV13 with high KCL solution (45 $\mathrm{mM} \mathrm{KCL}+10 \mathrm{mM} \mathrm{Ca}^{2+}$ ) followed by $\mathrm{NH}_{4} \mathrm{CL}$ solution to dequench all internal pHluorin signal, during imaging for 3 mins. We focused on axonal regions identified by signal of cotransfected synapsin-mCherry. We found an increase in fluorescence of pHluorin-Syt4 vesicles after high $\mathrm{KCl}$ addition and a further increase in intensity of fluorescence upon $\mathrm{NH}_{4} \mathrm{Cl}$ application, consistent with the literature for wild-type pHluorin-Syt4 responses (Dean et al., 2009; Dean et al., 2012), (Fig 3.18 A). Syt4-pHluorin responses showed an increase in fluorescence upon $\mathrm{KCl}$ stimulation, which remained elevated and plateaued for minutes after stimulation, indicating fusion of vesicles. Addition of $25 \mathrm{mM} \mathrm{NH}_{4} \mathrm{Cl}$ solution led to a further increase in fluorescence intensity that corresponded to the total internal vesicle content at synapses (Fig 3.18 B).

DCVs have been reported to fuse at both synaptic and non-synaptic sites in axons (van de Bospoort et al., 2012). Hence, we compared Syt4 pHluorin control and phosphomutant fusion properties at both synaptic and non-synaptic sites. Synaptic sites were marked by synapsin-mCherry and sites without synapsin-mCherry signal in axons were considered non-synaptic for analysis. At synaptic sites, delta F/F values of phosphomutants Syt4 pHluorin responses were very similar though control Syt4-pHluorin showed significantly more fluorescence upon $\mathrm{KCl}$ stimulation compared to either phosphomutant (Fig 3.18 C). 
A

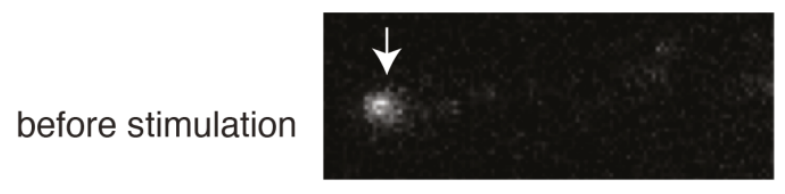

after $45 \mathrm{mM} \mathrm{KCl}$

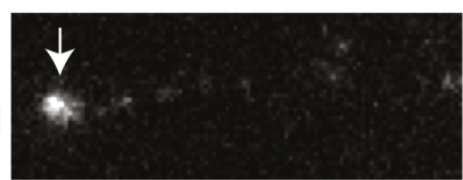

after $25 \mathrm{mM} \mathrm{NH}_{4} \mathrm{Cl}$

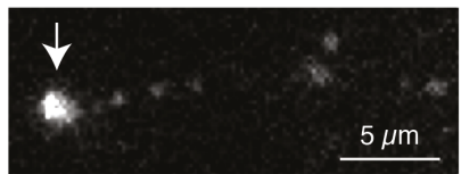

C

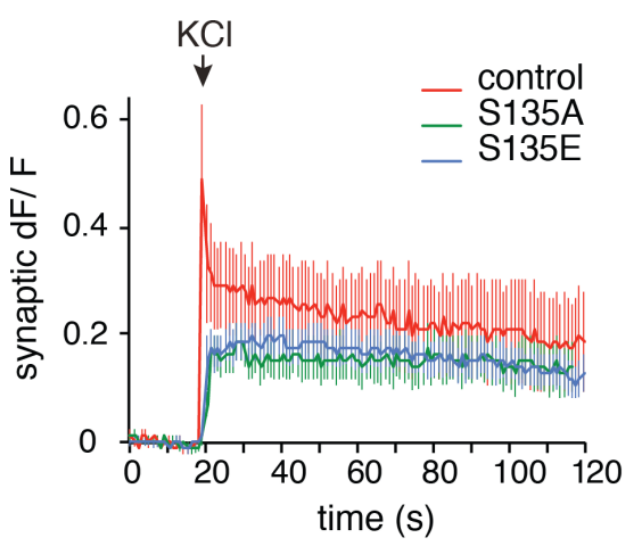

E

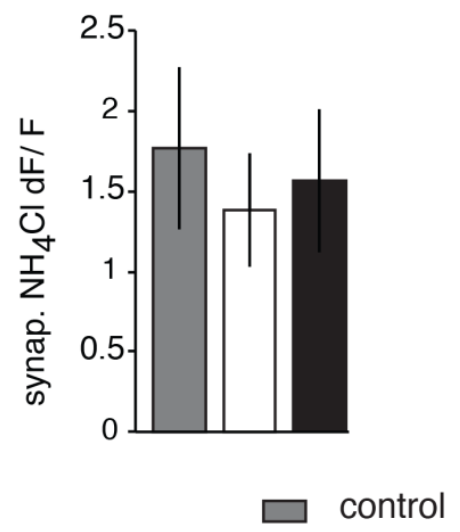

B

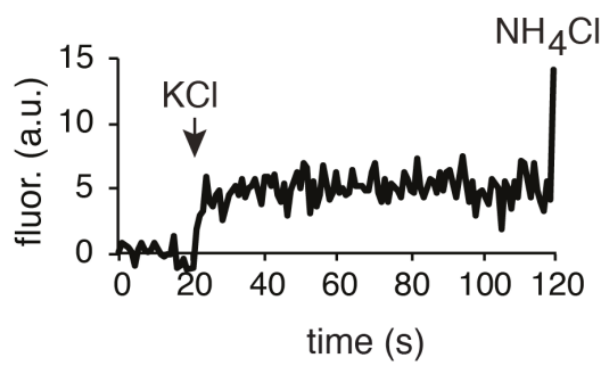

D

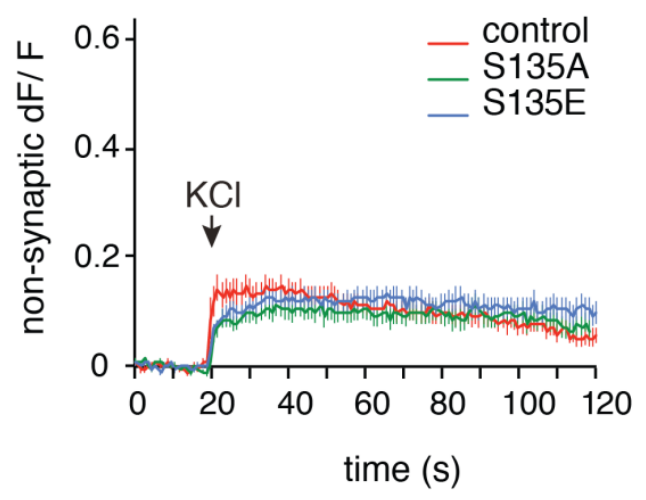

F

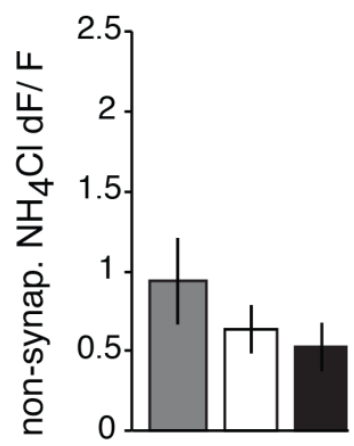

\section{S135E}

Figure 3.18 Fusion of phosphomutants S135A and S135E was similar at both synaptic and nonsynaptic sites in hippocampal neurons

(A) pHluorin-Syt4 exocytotic events (marked by white arrow) before stimulation, after $45 \mathrm{mM} \mathrm{KCl}$ and after $50 \mathrm{mM} \mathrm{NH}_{4} \mathrm{Cl}$ stimulation. Scale bar is $5 \mu \mathrm{m}$. (B) Sample trace of change in pHluorin-Syt4 fluorescence upon $\mathrm{KCl}$ stimulation and $\mathrm{NH}_{4} \mathrm{Cl}$ addition. (C) Average trace of $\mathrm{dF} / \mathrm{F}$ after $\mathrm{KCl}$ stimulation of control, $\mathrm{S} 135 \mathrm{~A}$ and S135E at synaptic and non-synaptic (D) sites. (E) Quantification of $\mathrm{dF} / \mathrm{F}$ of $\mathrm{NH}_{4} \mathrm{Cl}$ response at synaptic and non-synaptic (F) sites of control, S135A and S135E pHluorin-Syt4 ( $n=12,17$ and 21 events at synapses and $n=$ 34, 29 and 41 events at non-synaptic sites for control, S135A and S135E pHluorin-Syt4, respectively). Error indicates S.E.M. Significance was determined by Student's t-tests with Bonferroni correction. 
This difference in fusion characteristics of control with the phosphomutant vesicles at synaptic sites could be because phosphodeficient vesicles are highly mobile and might not get to synaptic sites, resulting in significantly less fusion. On the other hand, phosphomimetic Syt4 vesicles might get dropped at incorrect docking sites contributing to comparatively less fusion at synaptic sites.

On contrary, at non-synaptic sites all control, S135A and S135E Syt4 pHluorins had very similar kinetics of fusion (Fig 3.18 D). Synaptic and non-synaptic delta $\mathrm{F} / \mathrm{F}$ values upon $\mathrm{NH}_{4} \mathrm{Cl}$ stimulation of all three pHluorin contructs were also very similar, and were not significantly different. Together, these data shows that phosphorylation of Syt4 at S135 doesn't change the fusion of DCVs significantly at synaptic or non-synaptic sites in hippocampal neurons. 


\section{Discussion}

The question of whether and how transiting DCVs are recruited to pre-synaptic terminals in hippocampal neurons has remained an open question for many years. In this study, we examined these aspects of DCV trafficking in hippocampal neurons. We showed that a specific isoform of the synaptotagmin family, Syt4, specifically localises to DCVs and these Syt4 labelled DCVs undergo long range trafficking in neurons. In PC-12 cells, it was reported previously that c-Jun N-terminal kinase (JNK) phosphorylates Syt4 at the S135 site resulting in translocation of Syt4 from immature to mature secretory vesicles and enhances $\mathrm{Ca}^{2+}$ evoked release (Mori et al., 2008). Also, JNK has been shown to play a critical role in regulating vesicle mobility and in unloading cargoes from microtubular tracks in neurons (Stagi et al., 2006; Morfini et al., 2009; Gibbs et al., 2015). Consistent with the literature, we found that phosphorylation of Syt4 at S135 by JNK affects DCV transport in hippocampal neurons. Phosphorylated vesicles were less mobile and were more concentrated at presynaptic sites compared to non-phosphorylated vesicles in mature neurons. Furthermore, we tested if neuronal activity can lead to this phosphorylation effect, causing vesicle capture at synapses in mammalian hippocampal neurons, as occurs in Drosophila neurons (Shakiryanova et al., 2006; Bulgari et al., 2014). Indeed, we found that activity dependent JNK phosphorylation of Syt4 at the S135 site reduces DCV trafficking and promotes capture at synapses in hippocampal neurons. This study provides insights into the mechanisms of fast capture of DCVs at specific synapses in hippocampal neurons, which may subsequently lead to modification of synaptic functions, for example by the release of cargoes like BDNF from DCVs at potentiated synapses during learning and memory.

\subsection{Long range trafficking of Syt4 harboring dense core vesicles on microtubules of hippocampal neurons}

Syt4, a unique member of the synaptotagmin family of proteins is integral to brain derived neurotrophic factor (BDNF) containing DCVs in both axons and dendrites in hippocampal neurons (Dean et al., 2009; Dean et al., 2012). DCVs are known to efficiently co-package and co-transport different cargoes in hippocampal neurons (Lochner et al., 2008; Kwinter et al., 2009). Here we showed (Fig 3.1) Syt4 was localised to DCVs containing chromogranin A ( $\operatorname{CgA}) . \operatorname{CgA}$ is considered to be a major constituent of DCVs in endocrine and 
neuroendocrine cells and in neurons (Erickson et al., 1992; Adams et al., 1993; Kim et al., 2001; Machado et al., 2010). Hence, Syt4 marked the majority of DCVs in hippocampal neurons. In addition to this, our subcellular fractionation data and immuno-organelle isolation of SVs agrees with literature showing that Syt4 is absent from synaptic vesicles (Berton et al., 2000; Ibata et al., 2002).

In contrast to SVs, which are synthesised and recycled at synaptic sites, the biogenesis of DCVs is known to take place in the cell soma, from where they are then transported on microtubules into axons and dendrites (Burgoyne and Morgan, 2003; Kim et al., 2006; Wong et al., 2012). Depolymerizing MTs with nocodazole has been reported to disrupt BDNF containing DCV movement in neurons (Gauthier et al., 2004; Colin et al., 2008). We also found that these Syt4 labelled DCVs were highly mobile on microtubules and disrupting microtubules with nocodazole hampered vesicle trafficking (Fig 3.2 A and B).

DCVs have been reported to undergo long range trafficking and to be more mobile in axons compared to dendrites (Adachi et al., 2005; de Wit et al., 2006), and our results are consistent with these reports; we also showed that Syt4 labelled DCVs were transported long distance and were more mobile in axons compared to dendrites (Fig 3.6 and Fig 3.7 E panels). This difference in trafficking between axons and dendrites could be due to 1) the orientation of microtubules in axons versus dendrites, where microtubules have uniform polarity in axons, while dendrites have mixed polarity (Baas et al., 1988), or 2) MAP2 (microtubule associated protein 2), which is specifically present in dendrites and has been reported to interfere with kinesin based trafficking of vesicles (Drechsel et al., 1992).

\subsection{1. "Sushi-Model" of DCV trafficking in hippocampal neurons}

At the Drosophila neuromuscular junction, DCVs undergo a circuitous trafficking pattern termed the "sushi-model" in which they are routed from the cell soma to the distal tip of axons and back again to ensure delivery of cargoes to all boutons at which they are required (Moughamian and Holzbaur, 2012; Wong et al., 2012) - like sushi being delivered by conveyer belt to patrons at a sushi restaurant. Kinesin and dynein motor proteins drive DCVs filled with cargo in anterograde and retrograde directions. We found that this model also holds true in mammalian hippocampal axons (Fig 3.2 C and D). Although the long length of mammalian hippocampal axons precludes imaging an entire axon to track single vesicle trajectories in mature neurons, we imaged a total of approximately 1000 vesicles in 
approximately $5 \mathrm{~mm}$ of mid-axonal regions and around 200 vesicles in $1 \mathrm{~mm}$ of growth cone regions at DIV13. Strikingly, vesicles in mid-axonal regions only rarely switched directions and trafficked continually in either anterograde or retrograde directions, while in growth cones we observed many switches in direction of vesicles.

This might occur because mid-axonal regions contain a mix of labile and stable microtubule domains, while distal ends of axons consist almost entirely of labile domains (Baas and Black, 1990) characterized by tyrosinated alpha-tubulin (Ahmad, 1992). Also, we know that vesicles in neurons simultaneously carry both plus end directed kinesins and minus end directed dynein motors (Hancock, 2014) and activation of either of them determines the direction of travel, hence, it might be the activation of dynein motors on DCVs causing switching of directions near the growth cone. In addition, dynactin, a co-factor of dynein, is enriched in the distal end of axons and recruitment of this co-factor with other endbinding proteins initiates retrograde movement of vesicles (Moughamian and Holzbaur, 2012; Moughamian et al., 2013). Thus dynein present in an inactive state on vesicles might be specifically activated at the distal end of axons resulting in directionality switches of DCVs in hippocampal neurons.

\subsection{JNK, the kinase responsible for phosphorylating Syt4 at S135}

Several lines of evidence in this study indicate that JNK is the kinase that phosphorylates Syt4 at S135. In PC-12 cells, Syt4 has been already shown to get phosphorylated at the S135 site by JNK which enhances $\mathrm{Ca}^{2+}$ evoked release (Mori et al., 2008). Also, phosphoproteomics analysis by mass spectrometry of mouse brain in various studies has highlighted S135 as a site of Syt4 that is phosphorylated (Huttlin et al., 2010; Wisniewski et al., 2010; Goswami et al., 2012). We verified by in-silico analysis, in-vitro kinase assays that JNK phosphorylates Syt4 at this site. Co-IP experiments also showed the interaction of JNK with Syt4 (Fig 3.12). Furthermore in trafficking experiments, over-expression of active JNK1 made Syt4 vesicles less mobile, whereas dominant negative JNK1 made Syt4 vesicles more mobile than control. In addition, phospho-deficient S135A Syt4 vesicles remained highly mobile even in the presence of over-expressed active JNK1, indicating that JNK1 specifically phosphorylates Syt4 at the S135 site (Fig 3.14).

Although we focused on JNK1, it is possible that additional JNK isoforms play similar roles in DCV capture in neurons. For example, JNK3 phosphorylates KIF5C at S176, which reduces 
its ability to bind microtubules, and inhibits fast anterograde axonal transport in squid axoplasm (Morfini et al., 2006). While JNK1 is ubiquitously expressed, JNK3 is present in the hippocampus (Lee et al., 1999; Coffey, 2014) and could also play a role in vesicle capture. Activation of the JNK signaling cascade has been reported to inhibit axonal transport of mitochondria and synaptophysin by disrupting the interaction between kinesin-1 and microtubules in hippocampal neurons (Stagi et al., 2006). JNK also regulates fast axonal transport by acting on adaptor proteins like JNK-interacting protein 1 (JIP1). Phosphorylation of JIP1 at S421 increases anterograde transport and non-phosphorylated JIP1 favours retrograde transport of amyloid precursor protein (APP) vesicles (Fu and Holzbaur, 2013). Hence, phosphorylation of motor proteins, adaptor proteins or cargoes directly is important for regulating axonal transport (Gibbs et al., 2015).

\subsection{Phosphorylation of Syt4 at S135 decreases DCV trafficking in mature neurons}

In this study, we compared DCV trafficking of Syt4 phosphomutant vesicles, phosphodeficient Syt4 (S135A) and phosphomimetic Syt4 (S135E). with wild-type Syt4 vesicles and found that phosphorylated Syt4 vesicles have decreased mobility in mature hippocampal neuronal culture (Fig 3.4 and 3.5).

\subsubsection{Phosphorylation of Syt4 at S135 reduces DCV mobility developmentally}

At DIV6, before the majority of synapses have formed, Syt4 phosphomutants didn't show significant differences in DCV mobility parameters. At DIV13, after significantly high numbers of synapses are formed (Fig 3.3), we found that average speed and displacement/s of phosphodeficient S135A vesicles were similar to DIV6. However, mobility of control and phosphomimetic S135E vesicles were reduced significantly compared to at DIV6. These results indicate that mature hippocampal cultures at DIV13 have the ability to pause phosphorylated vesicles, resulting in decreased mobility of control and S135E vesicles whereas S135A vesicles, which can't be phosphorylated, show no change in trafficking. This effect of phosphorylation of Syt4 at S135 on trafficking was seen both in axons and dendrites even though we found significantly higher mobility of DCVs in axons compared to dendrites consistent with previous studies (Adachi et al., 2005; de Wit et al., 2006). 
We also found that p-JNK is more localised to synapses at DIV13 compared to DIV6 (Fig 3.16), which might contribute to the observed differences in trafficking of Syt4 phosphomutants vesicles developmentally.

\subsubsection{Phosphorylated Syt4 vesicles show reduced interaction with the motor protein Kif1A}

Kif1A is the motor protein responsible for anterograde DCV axonal transport in hippocampal neurons (Lo et al., 2011). Also in Drosophila and C. elegans, the homolog of Kif1A, unc-104, is required for axonal transport of DCVs (Bloom, 2001; Zahn et al., 2004; Barkus et al., 2008). We found in our experiments that the motor protein Kif1A cotransports with Syt4 vesicles in hippocampal neurons (Fig 3.9). In addition, Syt4 coimmunoprecipitates with Kif1A. We found that Syt4 S135E had reduced interaction with Kif1A compared to S135A and wild-type Syt4 (Fig 3.8). This destabilized interaction between Kif1A and vesicle cargo could result in reduced processivity by Kif1A (Soppina et al., 2014). Previous studies on kinesin-cargo release in the case of Kif17-Mint1 also showed that phosphorylation is an important mechanism to disrupt the binding of cargo to motor proteins, resulting in its release from microtubular tracks (Guillaud et al., 2008).

We performed Syt4 - Kif1A co-immunoprecipitation experiments in HEK293 cells. It would be interesting to compare the interaction of phosphomutants with Kif1A in neuronal cultures where additional adapter molecules that stabilize or destabilize cargo-motor binding, would be present. Various adaptor proteins are known to link cargoes with kinesin motors and regulate trafficking of vesicles, including liprin alpha, which binds Kif1A to traffic synaptic vesicle precursors, huntingtin which binds kinesin-1 to move BDNF vesicles, and JNK scaffolding proteins like JIP1 which binds kinesin-1 to transport amyloid precursor protein vesicles (Shin et al., 2003; Morfini et al., 2009; Goodwin and Juo, 2013; Fu and Holzbaur, 2013; Maday et al., 2014). It would be interesting to test if the decreased motility of S135E vesicles is because of reduced interaction with motors through adaptor proteins. We could also test if Syt4 phosphomutant vesicles have differences in interaction with microtubules themselves, which results in variability in trafficking parameters. MT associated proteins like tau regulate kinesin dependent trafficking by influencing the attachment and detachment of motors with MTs (Ebneth et al., 1998; Trinczek et al., 1999). 


\subsubsection{Over-expression of Kif1A rescues the trafficking of phosphorylated vesicles}

Over-expression of motor proteins has been shown to affect higher brain functions in vivo by mediating transportation of cargoes. For instance, Kif17 overexpression enhances learning and memory in mice by trafficking $\mathrm{N}$-methyl $\mathrm{D}$-aspartate type glutamate receptors (Wong et al., 2002). In addition, over-expressing Kif1A was shown to promote hippocampal synaptogenesis (Kondo et al., 2012).

Surprisingly, our results demonstrate that overexpressing Kif1A rescues the trafficking of S135E vesicles by enhancing their trafficking to the levels of S135A vesicles. Average speed and displacement of S135A and S135E with over-expressed Kif1A was almost identical suggesting that Syt4 motor protein binding sites are not saturated in control conditions with normal levels of Kif1A. Control vesicles trafficked significantly less compared to phosphomutants with Kif1A overexpression. Comparison of co-transport of Kif1A with Syt4 control and S135E vesicles showed that Kif1A co-trafficked significantly more with S135E vesicles compared to control Syt4 vesicles (Fig 3.9). Hence, it might be the lower number of Kif1A motors interacting with control Syt4 vesicles that leads to less trafficking of control Syt4 DCVs compared to phosphomutants with Kif1A overexpression.

\subsection{Activity dependent JNK phosphorylation captures Syt4 vesicles at synapses}

\subsubsection{Actin helps in capturing phosphorylated Syt4 vesicles at pre-synaptic sites}

We found that actin, which is highly enriched at pre-synaptic sites (Hirokawa et al., 1989; Cingolani and Goda, 2008), is necessary for the JNK-dependent capture of DCVs. Disruption of actin mobilizes phosphomimetic and control vesicles without showing any effect on phosphodeficient vesicles (Fig 3.11). This is consistent with previous reports that actin plays an important role in the mobility of DCVs in neuroendocrine cells (Oheim and Stuhmer, 2000; Lang et al., 2000).

Switching of cargo trafficking from microtubules to actin is known to be important for the delivery of cargoes to release sites (Heisler et al., 2011; Coles and Bradke, 2015). While kinesins and dyneins promote long-distance trafficking of organelles along microtubules, myosins drive cargo along actin for local transport from microtubules to the plasma membrane (Langford, 1995). The transition of cargo from microtubules to actin is 
suggested to occur through a "tug-of-war" between motor proteins, where the number of motors (i.e. the amount of force provided by each) can control cargo switching (Schroeder et al., 2010). Myosin Va interacts with DCVs (Bittins et al., 2009) and regulates their distribution and exocytosis in neuroendocrine cells as well as neurons (Rudolf, 2003; Kogel et al., 2010). If actin is disrupted as in our experiment, it might be that myosins cannot traffic "paused" vesicles locally into boutons via actin. This would cause kinesins to "win" via transportation on microtubules, resulting in increased motility of vesicles and defects in capture by actin at pre-synaptic sites. This data reveal that actin plays an important role in capturing phosphorylated vesicle at pre-synaptic sites.

\subsubsection{JNK dependent capture of Syt4 vesicles at active synapses}

We found that transiting Syt4 DCVs are captured at synapses by JNK-dependent phosphorylation. We examined the localisation of these Syt4 phosphomutants at synapses by fixed sample imaging and found that S135E was significantly more localised at presynaptic sites, but there was no difference at post-synaptic sites (Fig 3.10). Also, increasing active JNK by expression of MKK7-JNK1 caused Syt4 vesicles to accumulate at synapses (Fig 3.14 I). Fixed sample imaging may under-represent the localization of Syt4 S135E vesicles to synapses, since these vesicles are highly mobile.

The JNK-dependent capture of Syt4 DCVs was found to be activity-dependent (Fig 3.15), consistent with observations at the Drosophila NMJ (Shakiryanova et al., 2006; Bulgari et al., 2014). Pauses are defined according to (Wong et al., 2012) as immobility of a transiting vesicle at a site for greater than 120 seconds, while transient visits $(5-120 \mathrm{~s})$ and bypasses $(0-5 \mathrm{~s})$ were also reported in DCVs trafficking in Drosophila neurons. We found that Syt4 vesicles paused for 2 or more minutes in response to short term depolarization of neurons, while phosphodeficient Syt4 vesicles or Syt4 vesicles co-expressed with dominant negative JNK1 did not. These results showed that an increase in neuronal activity causes JNK dependent phosphorylation leading to capture of transiting vesicles.

In our pause analysis of trafficking experiments without inducing activity, pauses were defined as a deflection in average speed by $0.1 \mathrm{um} / \mathrm{s}$. Interestingly, we found that highly mobile vesicles (S135A, JNK1 (APF) or S135A/MKK7-JNK1 vesicles) pause more frequently but with shorter duration, whereas phosphorylated Syt4 vesicles have longer pause times but don't have a high number of pauses. This suggests that not all pauses are captures and 
capture is dependent on sufficient pause time and/or on the presence of motor proteins or other essential proteins involved in pausing, which may be heterogeous on DCV subpopulations (Bury and Sabo, 2011).

Furthermore, we found that $\mathrm{p}$-JNK was increased at synapses by increases in neuronal activity for 1 h., and this caused a concomitant increase in endogenous Syt4 at synapses, which was blocked by the JNK inhibitor SP600125 (Fig 3.17). A complete understanding of stimuli causing JNK activation at active synapses remains an open question. Interestingly, activation of NMDA receptors has been reported to increase neuronal activity and to activate MAP kinases, including JNK, in hippocampal neurons (Ko et al., 1998; Mukherjee et al., 1999). This NMDA-induced activation of JNK depended on calcium influx (Ko et al., 1998), which likely occurs predominantly at synapses. Calcium influx was also shown to be necessary for longer pause durations of synaptic vesicle protein transport vesicles at synapses (Sabo et al., 2006). Hence, it might be that calcium influx activates JNK leading to capture of DCVs at active synapses.

We observed significant decreases in DCV mobility caused by JNK1 phosphorylation, but vesicles are not completely immobilized. This could indicate additional capture mechanisms by other JNKs, or additional kinases, which could phosphorylate additional sites on Syt4 or on other DCV proteins. In C.elegans motor neurons, CaMKII-dependent phosphorylation was suggested as a mechanism to regulate transport and polarized distribution of DCVs by degrading the adaptor protein liprin-alpha (Hoogenraad et al., 2007; Goodwin and Juo, 2013). Liprin-alpha has been shown to interact with Kif1A (Shin et al., 2003); hence, it might be that CamKII and JNK act synergistically to regulate dense core vesicle distribution in mammalian neurons too.

The capture of vesicles we observed during short term stimulation were sometimes reversible, consistent with observations in Drosophila (Shakiryanova et al., 2006) and for Sema3A-GFP harboring dense core vesicles in mammalian neurons (de Wit et al., 2006). In the latter study these transient "capture" events correlated with increased Sema3A-GFP surface puncta, suggesting that transient capture may correspond to "kiss and run" transient fusion events (de Wit et al., 2006). 


\subsection{Effect of phosphorylating Syt4 at S135 on the fusion properties of DCVs}

DCVs are known to fuse at both synaptic and non-synaptic sites, though preferentially at synaptic sites in neurons (van de Bospoort et al., 2012). Syt4-pHluorin has been used as a marker to visualize exocytosis of DCVs in both axons and dendrites (Dean et al., 2009; Dean et al., 2012; Dean et al., 2012). We found, using Syt4 control and phosphomutant pHluorins, that Syt4 harboring vesicles fuse in response to high $\mathrm{KCl}$ stimulation in axons irrespective of Syt4 S135 phosphorylation state. At non-synaptic sites, we found no difference in fusion characteristics of control, S135A and S135E vesicles, indicating that the phosphorylation state of Syt4 S135 doesn't affect the fusion of vesicles at non-synaptic sites. On the contrary, on synaptic sites control Syt4 vesicles had a significantly higher magnitude of fusion compared to both phosphomutants upon $\mathrm{KCl}$ stimulation. S135A-pHluorin vesicles may exhibit less fusion because they are highly mobile, and hence, fewer vesicles are available for fusion at release sites. S135E-pHluorin vesicles, on the other hand, may show less fusion due to non-specific dropping off of S135E vesicles at sites away from docking and fusion sites. Proteins like Munc-13 and CAPS-1 aid in DCV fusion at docking sites. The absence of Munc-13 decreases the synaptic fusion rate of DCVs whereas overexpression has been reported to increase fusion events at non-synaptic sites, implying that Munc-13 might be necessary for priming/docking/fusion at synapses. CAPS-1 has also been reported to promote fusion of immobile or tethered DCVs at synaptic sites (van de Bospoort et al., 2012; Farina et al., 2015). Hence, if S135E vesicles were dropped too far from these docking sites, unloading from microtubules would not necessarily lead to more fusion. Interestingly, our findings that phosphomimetic vesicles were more localised to synaptic sites compared to phosphodeficient vesicles and that there is no difference in fusion between phosphomutants supports distinct roles of capture and fusion of vesicles at boutons, i.e. capture does not necessarily lead to fusion. This would also explain why there is more capture, but less fusion seen of phosphomimetic vesicles at synapse.

The percentage of vesicle fusion at individual sites calculated by $\mathrm{KCL} / \mathrm{NH}_{4} \mathrm{Cl}$ fluorescence also was not significantly different between control and phosphomutants at both synaptic and non-synaptic sites. But, interestingly, the total number of vesicles remaining after fusion present at synaptic sites was more than non-synaptic sites, consistent with EM data 
indicating 1-10 DCVs at synaptic sites (Sorra et al., 2006). Though studies comparing the number of DCVs at synaptic versus non-synaptic sites by EM are lacking. Quantitation of the numbers of DCVs at synaptic and non-synaptic sites would be informative to determine the distribution and efficiency of DCV release at each site.

Syt4 phosphorylation didn't change the fusion of DCVs in hippocampal neurons but other Syt isoforms or proteins may be present on DCVs to mediate fusion. Syt6, Syt7 and Syt9 exist on DCVs in PC-12 cells and also in hippocampal neurons raising the possibility that these isoforms may regulate DCV fusion in neurons (Zhang et al., 2011; Dean et al., 2012; Wong et al., 2015). Since Syt4 harbors an aspartate-to-serine substitution in its C2A domain leading to decreased $\mathrm{Ca}^{2+}$ sensitivity, it is probably not the key Syt isoform involved in fusion of DCVs but might be involved in $\mathrm{Ca}^{2+}$ independent functions (von Poser et al., 1997). Recently, Syt6 has been shown to trigger BDNF release in hippocampal neurons (Wong et al., 2015). According to our findings, Syt4 is at least involved in trafficking and capture of DCVs, and negatively regulates their fusion (Dean et al., 2009). Other Syt isoforms of proteins present on the same vesicles may promote the fusion of captured DCVs.

\subsection{Conclusions and future perspectives}

To summarize, we found that the majority of DCVs harbour the synaptotagmin isoform, Syt4 in hippocampal neurons. Trafficking of DCVs in dendrites was significantly less than in axons. In axons, these DCVs are highly mobile on microtubules and traffic circuitously as described by the "Sushi-model" first shown in the Drosophila NMJ (Moughamian and Holzbaur, 2012; Wong et al., 2012). Syt4-labelled DCVs traffic in a circuitous pattern, i.e either anterograde or retrograde directions and mainly switch directions at the distal end of axons near the growth cone. Following synapse formation, trafficking of DCVs harboring phosphorylated Syt4 decreases. In comparisons of phosphodeficient (S135A) and phosphomimetic (S135E) Syt4 phosphomutants we found that phosphorylated Syt4 vesicles have reduced motility and are more localised to pre-synaptic sites in mature neurons. Interestingly, the Kif1A motor protein showed less interaction with S135E vesicles, potentially explaining the reduced trafficking of phosphorylated vesicles. Actin was also necessary for the capture of phosphorylated vesicles in axons. Furthermore, by several lines of evidence, we showed that JNK is the kinase responsible for phosphorylating 
Syt4 at S135 and causing activity dependent reversible vesicle capture at synapses (Fig.

4.1).

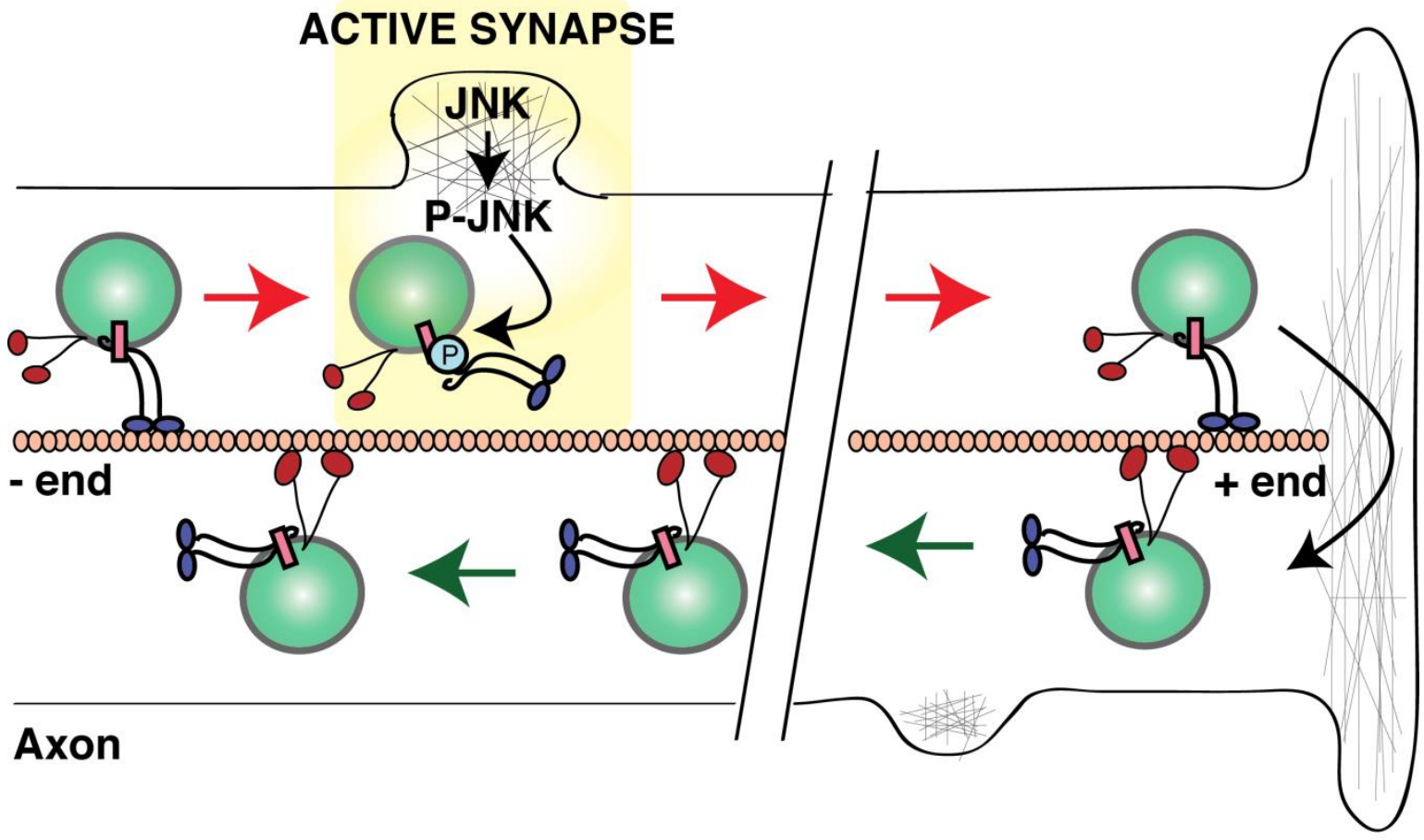

Figure Legend:

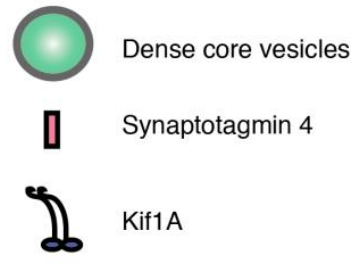

$00000000 \quad$ Microtubules

(P) Phosphorylation moeity
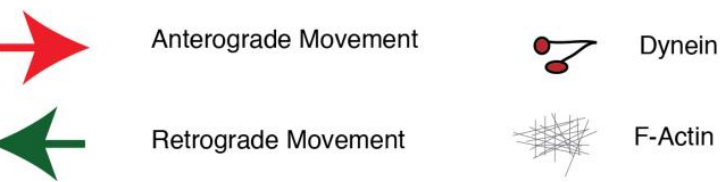

Figure 4.1 Proposed model for the capture of dense core vesicles by JNK dependent phosphorylation of Syt4 at S135 at active synapses in hippocampal neurons

Dense core vesicles undergo long range trafficking on microtubules via the microtubule associated motor protein Kif1A. These vesicles follow a circuitous trafficking pattern termed the "sushi model" in which switching of directions is mainly seen at the distal end of axons, near the growth cone. Syt4, a synaptotagmin isoform present specifically on DCVs interacts with Kif1A resulting in vesicle mobility. At active synapses, JNK phosphorylates Syt4 at S135 specifically. This phosphorylation causes reduced interaction of Syt4 on vesicles with the Kif1A motor protein and in turn decreased vesicle mobility. A decrease in vesicle mobility promotes capture of DCVs at active synapses. Actin, which is enriched at pre-synaptic sites, is necessary for capturing DCVs. This model enhances our understanding of activity dependent capture of DCVs from the transiting pool of vesicles in hippocampal neurons.

Although Syt4 phosphorylation at S135 was found to play a significant role in trafficking and capture of vesicles, there was no difference in the fusion of phosphodeficient vesicles 
compared to phosphomimetic Syt4 vesicles at synaptic or non-synaptic sites in hippocampal neurons.

Our study furthers the understanding of capture mechanisms of DCVs at active synapses in hippocampal neurons. The continual circuitous trafficking of DCVs in the axon and their activity-dependent capture at synaptic sites, provides a mechanism that allows for both fast and synapse-specific delivery of DCVs to release sites in neuronal processes. Future work is needed to understand the complete molecular machinery involved in precise spatial and temporal regulation of motor-cargo interactions important for DCV recruitment and fusion at release sites. Deducing the local signalling pathway at active pre-synaptic sites that causes JNK phosphorylation would aid in understanding the activity-dependent mechanism of DCV capture via JNK and its role in normal physiological conditions. Alterations in axonal transport, capture and fusion of DCVs has been implicated in neurodegenerative diseases, including Huntington's, Alzheimer's disease and autism (Chevalier-Larsen and Holzbaur, 2006; Sadakata and Furuichi, 2009; Dubey et al., 2015). Proper axonal transport and activity-dependent capture of DCVs is likely an important mechanism for efficient synaptic function. An understanding of the basic mechanisms of trafficking, capture and fusion of DCVs and how these mechanisms affect synaptic modulation and function may uncover novel strategies for combating neurodegenerative diseases in the future. 


\section{References}

Adachi, N., Kohara, K., and Tsumoto, T. (2005). Difference in trafficking of brain-derived neurotrophic factor between axons and dendrites of cortical neurons, revealed by live-cell imaging. BMC Neurosci 6, 42.

Adams, L.A., Ang, L.C., and Munoz, D.G. (1993). Chromogranin A, a soluble synaptic vesicle protein, is found in cortical neurons other than previously defined peptidergic neurons in the human neocortex. Brain research 602, 336-341.

Ahmad, B.a. (1992). The Plus Ends of Stable Microtubules Are the Exclusive Nucleating StructuresforMicrotubulesintheAxon.

Ahmed, S., Wittenmayer, N., Kremer, T., Hoeber, J., Kiran Akula, A., Urlaub, H., Islinger, M., Kirsch, J., Dean, C., and Dresbach, T. (2013). Mover is a homomeric phospho-protein present on synaptic vesicles. PLoS One 8, e63474.

Al-Bassam, J., Cui, Y., Klopfenstein, D., Carragher, B.O., Vale, R.D., and Milligan, R.A. (2003). Distinct conformations of the kinesin Unc104 neck regulate a monomer to dimer motor transition. J Cell Biol 163, 743-753.

Alabi, A.A., and Tsien, R.W. (2012). Synaptic vesicle pools and dynamics. Cold Spring Harb Perspect Biol 4, a013680.

Ali, M.Y., Krementsova, E.B., Kennedy, G.G., Mahaffy, R., Pollard, T.D., Trybus, K.M., and Warshaw, D.M. (2007). Myosin Va maneuvers through actin intersections and diffuses along microtubules. Proc Natl Acad Sci U S A 104, 4332-4336.

Arthur, C.P., Dean, C., Pagratis, M., Chapman, E.R., and Stowell, M.H. (2010). Loss of synaptotagmin IV results in a reduction in synaptic vesicles and a distortion of the Golgi structure in cultured hippocampal neurons. Neuroscience 167, 135-142.

Baas, P.W., and Black, M.M. (1990). Individual microtubules in the axon consist of domains that differ in both composition and stability. J Cell Biol 111, 495-509.

Baas, P.W., Deitch, J.S., Black, M.M., and Banker, G.A. (1988). Polarity orientation of microtubules in hippocampal neurons: uniformity in the axon and nonuniformity in the dendrite. Proc Natl Acad Sci U S A 85, 8335-8339.

Banker, G.A., and Cowan, W.M. (1977). Rat hippocampal neurons in dispersed cell culture. Brain research 126, 397-342.

Barkus, R.V., Klyachko, O., Horiuchi, D., Dickson, B.J., and Saxton, W.M. (2008). Identification of an axonal kinesin-3 motor for fast anterograde vesicle transport that facilitates retrograde transport of neuropeptides. Mol Biol Cell 19, 274-283.

Basu, J., Betz, A., Brose, N., and Rosenmund, C. (2007). Munc13-1 C1 domain activation lowers the energy barrier for synaptic vesicle fusion. J Neurosci 27, 1200-1210. 
Bean, A.J., Zhang, X., and Hokfelt, T. (1994). Peptide secretion: what do we know? Faseb j $8,630-638$.

Behrens, A., Sibilia, M., and Wagner, E.F. (1999). Amino-terminal phosphorylation of c-Jun regulates stress-induced apoptosis and cellular proliferation. Nature genetics 21, 326-329.

Bennett, B.L., Sasaki, D.T., Murray, B.W., O'Leary, E.C., Sakata, S.T., Xu, W., Leisten, J.C., Motiwala, A., Pierce, S., Satoh, Y., et al. (2001). SP600125, an anthrapyrazolone inhibitor of Jun N-terminal kinase. Proc Natl Acad Sci U S A 98, 13681-13686.

Berton, F., Cornet, V., Iborra, C., Garrido, J., Dargent, B., Fukuda, M., Seagar, M., and Marqueze, B. (2000). Synaptotagmin I and IV define distinct populations of neuronal transport vesicles. The European journal of neuroscience 12, 1294-1302.

Berton, F., Iborra, C., Boudier, J.A., Seagar, M.J., and Marqueze, B. (1997). Developmental regulation of synaptotagmin I, II, III, and IV mRNAs in the rat CNS. J Neurosci 17, 1206-1216.

Bhalla, A., Chicka, M.C., and Chapman, E.R. (2008). Analysis of the synaptotagmin family during reconstituted membrane fusion. Uncovering a class of inhibitory isoforms. J Biol Chem 283, 21799-21807.

Bhatnagar, Y.M. (1996). Immunocytochemical methods and protocols Lorette C. Javois, editor. Methods in Molecular Biology, Vol. 34, Humana Press, Totowa, NJ, 1994. American Journal of Medical Genetics 62, 202-202.

Biffi, E., Regalia, G., Menegon, A., Ferrigno, G., and Pedrocchi, A. (2013). The influence of neuronal density and maturation on network activity of hippocampal cell cultures: a methodological study. PLoS One 8, e83899.

Bittins, C.M., Eichler, T.W., and Gerdes, H.H. (2009). Expression of the dominant-negative tail of myosin Va enhances exocytosis of large dense core vesicles in neurons. Cell Mol Neurobiol 29, 597-608.

Bloom, G.S. (2001). The UNC-104/KIF1 family of kinesins. Curr Opin Cell Biol 13, 36-40.

Bogoyevitch, M.A., and Kobe, B. (2006). Uses for JNK: the Many and Varied Substrates of the c-Jun N-Terminal Kinases. Microbiology and Molecular Biology Reviews 70, 1061-1095.

Borgonovo, B., Ouwendijk, J., and Solimena, M. (2006). Biogenesis of secretory granules. Curr Opin Cell Biol 18, 365-370.

Brecht, S., Kirchhof, R., Chromik, A., Willesen, M., Nicolaus, T., Raivich, G., Wessig, J., Waetzig, V., Goetz, M., Claussen, M., et al. (2005). Specific pathophysiological functions of JNK isoforms in the brain. The European journal of neuroscience 21, 363-377.

Bulgari, D., Zhou, C., Hewes, R.S., Deitcher, D.L., and Levitan, E.S. (2014). Vesicle capture, not delivery, scales up neuropeptide storage in neuroendocrine terminals. Proc Natl Acad Sci U S A 111, 3597-3601. 
Burgoyne, R.D., and Morgan, A. (2003). Secretory granule exocytosis. Physiol Rev 83, 581632.

Burns, M.E., and Augustine, G.J. (1995). Synaptic structure and function: dynamic organization yields architectural precision. Cell 83, 187-194.

Bury, L.A., and Sabo, S.L. (2011). Coordinated trafficking of synaptic vesicle and active zone proteins prior to synapse formation. Neural Dev 6, 24.

Chevalier-Larsen, E., and Holzbaur, E.L. (2006). Axonal transport and neurodegenerative disease. Biochim Biophys Acta 1762, 1094-1108.

Cingolani, L.A., and Goda, Y. (2008). Actin in action: the interplay between the actin cytoskeleton and synaptic efficacy. Nat Rev Neurosci 9, 344-356.

Coffey, E.T. (2014). Nuclear and cytosolic JNK signalling in neurons. Nat Rev Neurosci 15, 285-299.

Coles, C.H., and Bradke, F. (2015). Coordinating neuronal actin-microtubule dynamics. Curr Biol 25, R677-691.

Colin, E., Zala, D., Liot, G., Rangone, H., Borrell-Pages, M., Li, X.J., Saudou, F., and Humbert, S. (2008). Huntingtin phosphorylation acts as a molecular switch for anterograde/retrograde transport in neurons. Embo j 27, 2124-2134.

Curtin, J.F., and Cotter, T.G. (2002). Anisomycin activates JNK and sensitises DU 145 prostate carcinoma cells to Fas mediated apoptosis. Br J Cancer 87, 1188-1194.

Davis, R.J. (2000). Signal transduction by the JNK group of MAP kinases. Cell 103, 239-252.

De Camilli, P., and Jahn, R. (1990). Pathways to regulated exocytosis in neurons. Annual review of physiology 52, 625-645.

de Wit, J., Toonen, R.F., Verhaagen, J., and Verhage, M. (2006). Vesicular trafficking of semaphorin $3 \mathrm{~A}$ is activity-dependent and differs between axons and dendrites. Traffic 7, 1060-1077.

Dean, C., Dunning, F.M., Liu, H., Bomba-Warczak, E., Martens, H., Bharat, V., Ahmed, S., and Chapman, E.R. (2012). Axonal and dendritic synaptotagmin isoforms revealed by a pHluorin-syt functional screen. Mol Biol Cell 23, 1715-1727.

Dean, C., Liu, H., Dunning, F.M., Chang, P.Y., Jackson, M.B., and Chapman, E.R. (2009). Synaptotagmin-IV modulates synaptic function and long-term potentiation by regulating BDNF release. Nat Neurosci 12, 767-776.

Dean, C., Liu, H., Staudt, T., Stahlberg, M.A., Vingill, S., Buckers, J., Kamin, D., Engelhardt, J., Jackson, M.B., Hell, S.W., et al. (2012). Distinct subsets of Syt-IV/BDNF vesicles are sorted to axons versus dendrites and recruited to synapses by activity. J Neurosci 32, 5398-5413. 
Derijard, B., Hibi, M., Wu, I.H., Barrett, T., Su, B., Deng, T., Karin, M., and Davis, R.J. (1994). JNK1: a protein kinase stimulated by UV light and Ha-Ras that binds and phosphorylates the c-Jun activation domain. Cell 76, 1025-1037.

Dieni, S., Matsumoto, T., Dekkers, M., Rauskolb, S., Ionescu, M.S., Deogracias, R., Gundelfinger, E.D., Kojima, M., Nestel, S., Frotscher, M., et al. (2012). BDNF and its propeptide are stored in presynaptic dense core vesicles in brain neurons. J Cell Biol 196, 775788.

Drechsel, D.N., Hyman, A.A., Cobb, M.H., and Kirschner, M.W. (1992). Modulation of the dynamic instability of tubulin assembly by the microtubule-associated protein tau. Molecular Biology of the Cell 3, 1141-1154.

Dubey, J., Ratnakaran, N., and Koushika, S.P. (2015). Neurodegeneration and microtubule dynamics: death by a thousand cuts. Front Cell Neurosci 9, 343.

Eaton, B.A., Haugwitz, M., Lau, D., and Moore, H.P. (2000). Biogenesis of regulated exocytotic carriers in neuroendocrine cells. J Neurosci 20, 7334-7344.

Ebneth, A., Godemann, R., Stamer, K., Illenberger, S., Trinczek, B., and Mandelkow, E. (1998). Overexpression of tau protein inhibits kinesin-dependent trafficking of vesicles, mitochondria, and endoplasmic reticulum: implications for Alzheimer's disease. J Cell Biol 143, 777-794.

Eilers, U., Klumperman, J., and Hauri, H.P. (1989). Nocodazole, a microtubule-active drug, interferes with apical protein delivery in cultured intestinal epithelial cells (Caco-2). J Cell Biol 108, 13-22.

Erickson, J.D., Lloyd, R., Trojanowski, J.Q., Iacangelo, A., and Eiden, E. (1992). Sites of synthesis of chromogranins A and B in the human brain. Neuropeptides 21, 239-244.

Farina, M., van de Bospoort, R., He, E., Persoon, C.M., van Weering, J.R., Broeke, J.H., Verhage, M., and Toonen, R.F. (2015). CAPS-1 promotes fusion competence of stationary dense-core vesicles in presynaptic terminals of mammalian neurons. Elife 4 .

Ferguson, G.D., Anagnostaras, S.G., Silva, A.J., and Herschman, H.R. (2000). Deficits in memory and motor performance in synaptotagmin IV mutant mice. Proc Natl Acad Sci U S A 97, 5598-5603.

Ferguson, G.D., Wang, H., Herschman, H.R., and Storm, D.R. (2004). Altered hippocampal short-term plasticity and associative memory in synaptotagmin IV (-/-) mice. Hippocampus 14, 964-974.

Franker, M.A., and Hoogenraad, C.C. (2013). Microtubule-based transport - basic mechanisms, traffic rules and role in neurological pathogenesis. J Cell Sci 126, 2319-2329.

Frey, U., Krug, M., Reymann, K.G., and Matthies, H. (1988). Anisomycin, an inhibitor of protein synthesis, blocks late phases of LTP phenomena in the hippocampal CA1 region in vitro. Brain research 452, 57-65. 
Fu, M.M., and Holzbaur, E.L. (2013). JIP1 regulates the directionality of APP axonal transport by coordinating kinesin and dynein motors. J Cell Biol 202, 495-508.

Fykse, E.M., and Fonnum, F. (1988). Uptake of gamma-aminobutyric acid by a synaptic vesicle fraction isolated from rat brain. J Neurochem 50, 1237-1242.

Gauthier, L.R., Charrin, B.C., Borrell-Pages, M., Dompierre, J.P., Rangone, H., Cordelieres, F.P., De Mey, J., MacDonald, M.E., Lessmann, V., Humbert, S., et al. (2004). Huntingtin controls neurotrophic support and survival of neurons by enhancing BDNF vesicular transport along microtubules. Cell 118, 127-138.

Gibbs, K.L., Greensmith, L., and Schiavo, G. (2015). Regulation of Axonal Transport by Protein Kinases. Trends Biochem Sci 40, 597-610.

Goldstein, L.S., and Yang, Z. (2000). Microtubule-based transport systems in neurons: the roles of kinesins and dyneins. Annu Rev Neurosci 23, 39-71.

Gondre-Lewis, M.C., Park, J.J., and Loh, Y.P. (2012). Cellular mechanisms for the biogenesis and transport of synaptic and dense-core vesicles. Int Rev Cell Mol Biol 299, 27-115.

Goodwin, P.R., and Juo, P. (2013). The scaffolding protein SYD-2/Liprin-alpha regulates the mobility and polarized distribution of dense-core vesicles in C. elegans motor neurons. PLoS One 8, e54763.

Gorr, S.U., Jain, R.K., Kuehn, U., Joyce, P.B., and Cowley, D.J. (2001). Comparative sorting of neuroendocrine secretory proteins: a search for common ground in a mosaic of sorting models and mechanisms. Molecular and cellular endocrinology 172, 1-6.

Goswami, T., Li, X., Smith, A.M., Luderowski, E.M., Vincent, J.J., Rush, J., and Ballif, B.A. (2012). Comparative phosphoproteomic analysis of neonatal and adult murine brain. Proteomics 12, 2185-2189.

Guillaud, L., Wong, R., and Hirokawa, N. (2008). Disruption of KIF17-Mint1 interaction by CaMKII-dependent phosphorylation: a molecular model of kinesin-cargo release. Nat Cell Biol 10, 19-29.

Guzik, B.W., and Goldstein, L.S. (2004). Microtubule-dependent transport in neurons: steps towards an understanding of regulation, function and dysfunction. Curr Opin Cell Biol 16, 443-450.

Hall, D.H., and Hedgecock, E.M. (1991). Kinesin-related gene unc-104 is required for axonal transport of synaptic vesicles in C. elegans. Cell 65, 837-847.

Hammond, J.W., Cai, D., Blasius, T.L., Li, Z., Jiang, Y., Jih, G.T., Meyhofer, E., and Verhey, K.J. (2009). Mammalian Kinesin-3 motors are dimeric in vivo and move by processive motility upon release of autoinhibition. PLoS Biol 7, e72.

Hancock, W.O. (2014). Bidirectional cargo transport: moving beyond tug of war. Nat Rev Mol Cell Biol 15, 615-628. 
Heisler, F.F., Loebrich, S., Pechmann, Y., Maier, N., Zivkovic, A.R., Tokito, M., Hausrat, T.J., Schweizer, M., Bahring, R., Holzbaur, E.L., et al. (2011). Muskelin regulates actin filamentand microtubule-based GABA(A) receptor transport in neurons. Neuron 70, 66-81.

Heuser, J.E., and Reese, T.S. (1973). Evidence for recycling of synaptic vesicle membrane during transmitter release at the frog neuromuscular junction. J Cell Biol 57, 315-344.

Heyer, E.J., Nowak, L.M., and Macdonald, R.L. (1982). Membrane depolarization and prolongation of calcium-dependent action potentials of mouse neurons in cell culture by two convulsants: bicuculline and penicillin. Brain research 232, 41-56.

Hirokawa, N. (1998). Kinesin and dynein superfamily proteins and the mechanism of organelle transport. Science $279,519-526$.

Hirokawa, N., Niwa, S., and Tanaka, Y. (2010). Molecular motors in neurons: transport mechanisms and roles in brain function, development, and disease. Neuron 68, 610-638.

Hirokawa, N., Niwa, S., and Tanaka, Y. (2010). Molecular Motors in Neurons: Transport Mechanisms and Roles in Brain Function, Development, and Disease. Neuron 68, 610-638.

Hirokawa, N., Sobue, K., Kanda, K., Harada, A., and Yorifuji, H. (1989). The cytoskeletal architecture of the presynaptic terminal and molecular structure of synapsin 1. J Cell Biol 108, 111-126.

Hodgkin, A.L., and Huxley, A.F. (1952). A quantitative description of membrane current and its application to conduction and excitation in nerve. J Physiol 117, 500-544.

Hoogenraad, C.C., Feliu-Mojer, M.I., Spangler, S.A., Milstein, A.D., Dunah, A.W., Hung, A.Y., and Sheng, M. (2007). Liprinalpha1 degradation by calcium/calmodulin-dependent protein kinase II regulates LAR receptor tyrosine phosphatase distribution and dendrite development. Dev Cell 12, 587-602.

Hormuzdi, S.G., Filippov, M.A., Mitropoulou, G., Monyer, H., and Bruzzone, R. (2004). Electrical synapses: a dynamic signaling system that shapes the activity of neuronal networks. Biochim Biophys Acta 1662, 113-137.

Howard, J., and Hyman, A.A. (2009). Growth, fluctuation and switching at microtubule plus ends. Nat Rev Mol Cell Biol 10, 569-574.

Huo, L., Yue, Y., Ren, J., Yu, J., Liu, J., Yu, Y., Ye, F., Xu, T., Zhang, M., and Feng, W. (2012). The CC1-FHA tandem as a central hub for controlling the dimerization and activation of kinesin-3 KIF1A. Structure 20, 1550-1561.

Huttlin, E.L., Jedrychowski, M.P., Elias, J.E., Goswami, T., Rad, R., Beausoleil, S.A., Villen, J., Haas, W., Sowa, M.E., and Gygi, S.P. (2010). A tissue-specific atlas of mouse protein phosphorylation and expression. Cell 143, 1174-1189.

Ibata, K., Fukuda, M., Hamada, T., Kabayama, H., and Mikoshiba, K. (2000). Synaptotagmin IV is present at the Golgi and distal parts of neurites. J Neurochem 74, 518-526. 
Ibata, K., Hashikawa, T., Tsuboi, T., Terakawa, S., Liang, F., Mizutani, A., Fukuda, M., and Mikoshiba, K. (2002). Non-polarized distribution of synaptotagmin IV in neurons: evidence that synaptotagmin IV is not a synaptic vesicle protein. Neurosci Res 43, 401-406.

Ichikawa, M., Muramoto, K., Kobayashi, K., Kawahara, M., and Kuroda, Y. (1993). Formation and maturation of synapses in primary cultures of rat cerebral cortical cells: an electron microscopic study. Neurosci Res 16, 95-103.

Ikeda, K., and Bekkers, J.M. (2009). Counting the number of releasable synaptic vesicles in a presynaptic terminal. Proc Natl Acad Sci U S A 106, 2945-2950.

Ip, Y.T., and Davis, R.J. (1998). Signal transduction by the c-Jun N-terminal kinase (JNK)-from inflammation to development. Curr Opin Cell Biol 10, 205-219.

Jessell, T.M., and Kandel, E.R. (1993). Synaptic transmission: a bidirectional and selfmodifiable form of cell-cell communication. Cell 72 Suppl, 1-30.

Jin, Y., and Garner, C.C. (2008). Molecular mechanisms of presynaptic differentiation. Annu Rev Cell Dev Biol 24, 237-262.

Jung, N., and Haucke, V. (2007). Clathrin-mediated endocytosis at synapses. Traffic 8, 11291136.

Kapitein, L.C., and Hoogenraad, C.C. (2011). Which way to go? Cytoskeletal organization and polarized transport in neurons. Mol Cell Neurosci 46, 9-20.

Kapitein, L.C., and Hoogenraad, C.C. (2015). Building the Neuronal Microtubule Cytoskeleton. Neuron 87, 492-506.

Kardon, J.R., and Vale, R.D. (2009). Regulators of the cytoplasmic dynein motor. Nat Rev Mol Cell Biol 10, 854-865.

Karki, S., and Holzbaur, E.L. (1999). Cytoplasmic dynein and dynactin in cell division and intracellular transport. Curr Opin Cell Biol 11, 45-53.

Kelly, R.B. (1991). Secretory granule and synaptic vesicle formation. Curr Opin Cell Biol 3, 654-660.

Kim, J.H., Lee, S.R., Li, L.H., Park, H.J., Park, J.H., Lee, K.Y., Kim, M.K., Shin, B.A., and Choi, S.Y. (2011). High cleavage efficiency of a $2 A$ peptide derived from porcine teschovirus- 1 in human cell lines, zebrafish and mice. PLoS One 6, e18556.

Kim, T., Gondre-Lewis, M.C., Arnaoutova, I., and Loh, Y.P. (2006). Dense-core secretory granule biogenesis. Physiology (Bethesda, Md) 21, 124-133.

Kim, T., Tao-Cheng, J.H., Eiden, L.E., and Loh, Y.P. (2001). Chromogranin A, an "on/off" switch controlling dense-core secretory granule biogenesis. Cell 106, 499-509. 
Kleele, T., Marinkovic, P., Williams, P.R., Stern, S., Weigand, E.E., Engerer, P., Naumann, R., Hartmann, J., Karl, R.M., Bradke, F., et al. (2014). An assay to image neuronal microtubule dynamics in mice. Nat Commun 5, 4827.

Klopfenstein, D.R., Tomishige, M., Stuurman, N., and Vale, R.D. (2002). Role of phosphatidylinositol $(4,5)$ bisphosphate organization in membrane transport by the Unc104 kinesin motor. Cell 109, 347-358.

Kneussel, M., and Wagner, W. (2013). Myosin motors at neuronal synapses: drivers of membrane transport and actin dynamics. Nat Rev Neurosci 14, 233-247.

Ko, H.W., Park, K.Y., Kim, H., Han, P.L., Kim, Y.U., Gwag, B.J., and Choi, E.J. (1998). Ca2+mediated activation of $\mathrm{c}$-Jun $\mathrm{N}$-terminal kinase and nuclear factor kappa $\mathrm{B}$ by NMDA in cortical cell cultures. J Neurochem 71, 1390-1395.

Kogel, T., Bittins, C.M., Rudolf, R., and Gerdes, H.H. (2010). Versatile roles for myosin Va in dense core vesicle biogenesis and function. Biochem Soc Trans 38, 199-204.

Kondo, M., Takei, Y., and Hirokawa, N. (2012). Motor protein KIF1A is essential for hippocampal synaptogenesis and learning enhancement in an enriched environment. Neuron 73, 743-757.

Kononenko, N.L., and Haucke, V. (2015). Molecular mechanisms of presynaptic membrane retrieval and synaptic vesicle reformation. Neuron 85, 484-496.

Kumar, S., Lee, I.H., and Plamann, M. (2000). Cytoplasmic dynein ATPase activity is regulated by dynactin-dependent phosphorylation. J Biol Chem 275, 31798-31804.

Kwan, A.C., Dombeck, D.A., and Webb, W.W. (2008). Polarized microtubule arrays in apical dendrites and axons. Proc Natl Acad Sci U S A 105, 11370-11375.

Kwinter, D.M., Lo, K., Mafi, P., and Silverman, M.A. (2009). Dynactin regulates bidirectional transport of dense-core vesicles in the axon and dendrites of cultured hippocampal neurons. Neuroscience 162, 1001-1010.

Kyriakis, J.M., and Avruch, J. (2012). Mammalian MAPK signal transduction pathways activated by stress and inflammation: a 10-year update. Physiol Rev 92, 689-737.

Lang, T., Wacker, I., Wunderlich, I., Rohrbach, A., Giese, G., Soldati, T., and Almers, W. (2000). Role of actin cortex in the subplasmalemmal transport of secretory granules in PC12 cells. Biophys J 78, 2863-2877.

Langford, G.M. (1995). Actin- and microtubule-dependent organelle motors: interrelationships between the two motility systems. Curr Opin Cell Biol 7, 82-88.

Lawrence, C.J., Dawe, R.K., Christie, K.R., Cleveland, D.W., Dawson, S.C., Endow, S.A., Goldstein, L.S., Goodson, H.V., Hirokawa, N., Howard, J., et al. (2004). A standardized kinesin nomenclature. J Cell Biol 167, 19-22. 
Lee, J.-R., Shin, H., Ko, J., Choi, J., Lee, H., and Kim, E. (2003). Characterization of the Movement of the Kinesin Motor KIF1A in Living Cultured Neurons. Journal of Biological Chemistry 278, 2624-2629.

Lee, J.K., Park, J., Lee, Y.D., Lee, S.H., and Han, P.L. (1999). Distinct localization of SAPK isoforms in neurons of adult mouse brain implies multiple signaling modes of SAPK pathway. Brain research Molecular brain research 70, 116-124.

Lee, J.R., Shin, H., Ko, J., Choi, J., Lee, H., and Kim, E. (2003). Characterization of the movement of the kinesin motor KIF1A in living cultured neurons. J Biol Chem 278, 26242629.

Lei, K., Nimnual, A., Zong, W.X., Kennedy, N.J., Flavell, R.A., Thompson, C.B., Bar-Sagi, D., and Davis, R.J. (2002). The Bax Subfamily of Bcl2-Related Proteins Is Essential for Apoptotic Signal Transduction by c-Jun NH2-Terminal Kinase. Molecular and Cellular Biology 22, 49294942.

Lessmann, V., Gottmann, K., and Malcangio, M. (2003). Neurotrophin secretion: current facts and future prospects. Progress in neurobiology 69, 341-374.

Littleton, J.T., Serano, T.L., Rubin, G.M., Ganetzky, B., and Chapman, E.R. (1999). Synaptic function modulated by changes in the ratio of synaptotagmin I and IV. Nature 400, 757760.

Lo, K.Y., Kuzmin, A., Unger, S.M., Petersen, J.D., and Silverman, M.A. (2011). KIF1A is the primary anterograde motor protein required for the axonal transport of dense-core vesicles in cultured hippocampal neurons. Neurosci Lett 491, 168-173.

Lochner, J.E., Spangler, E., Chavarha, M., Jacobs, C., McAllister, K., Schuttner, L.C., and Scalettar, B.A. (2008). Efficient copackaging and cotransport yields postsynaptic colocalization of neuromodulators associated with synaptic plasticity. Dev Neurobiol 68, 1243-1256.

Machado, H.B., Liu, W., Vician, L.J., and Herschman, H.R. (2004). Synaptotagmin IV overexpression inhibits depolarization-induced exocytosis in PC12 cells. Journal of neuroscience research 76, 334-341.

Machado, J.D., Diaz-Vera, J., Dominguez, N., Alvarez, C.M., Pardo, M.R., and Borges, R. (2010). Chromogranins A and B as regulators of vesicle cargo and exocytosis. Cell Mol Neurobiol 30, 1181-1187.

Maday, S., Twelvetrees, A.E., Moughamian, A.J., and Holzbaur, E.L. (2014). Axonal transport: cargo-specific mechanisms of motility and regulation. Neuron 84, 292-309.

Maeder, C.I., Shen, K., and Hoogenraad, C.C. (2014). Axon and dendritic trafficking. Curr Opin Neurobiol 27, 165-170.

Matsuda, N., Lu, H., Fukata, Y., Noritake, J., Gao, H., Mukherjee, S., Nemoto, T., Fukata, M., and Poo, M.M. (2009). Differential activity-dependent secretion of brain-derived neurotrophic factor from axon and dendrite. J Neurosci 29, 14185-14198. 
Maycox, P.R., Deckwerth, T., Hell, J.W., and Jahn, R. (1988). Glutamate uptake by brain synaptic vesicles. Energy dependence of transport and functional reconstitution in proteoliposomes. J Biol Chem 263, 15423-15428.

Mcllvain, J.M., Jr., Burkhardt, J.K., Hamm-Alvarez, S., Argon, Y., and Sheetz, M.P. (1994). Regulation of kinesin activity by phosphorylation of kinesin-associated proteins. J Biol Chem 269, 19176-19182.

McMahon, H.T., Bolshakov, V.Y., Janz, R., Hammer, R.E., Siegelbaum, S.A., and Sudhof, T.C. (1996). Synaptophysin, a major synaptic vesicle protein, is not essential for neurotransmitter release. Proc Natl Acad Sci U S A 93, 4760-4764.

Miesenbock, G., De Angelis, D.A., and Rothman, J.E. (1998). Visualizing secretion and synaptic transmission with $\mathrm{pH}$-sensitive green fluorescent proteins. Nature 394, 192-195.

Morfini, G., Pigino, G., Szebenyi, G., You, Y., Pollema, S., and Brady, S.T. (2006). JNK mediates pathogenic effects of polyglutamine-expanded androgen receptor on fast axonal transport. Nat Neurosci 9, 907-916.

Morfini, G.A., You, Y.M., Pollema, S.L., Kaminska, A., Liu, K., Yoshioka, K., Bjorkblom, B., Coffey, E.T., Bagnato, C., Han, D., et al. (2009). Pathogenic huntingtin inhibits fast axonal transport by activating JNK3 and phosphorylating kinesin. Nat Neurosci 12, 864-871.

Mori, Y., and Fukuda, M. (2011). Synaptotagmin IV acts as a multi-functional regulator of Ca2+-dependent exocytosis. Neurochem Res 36, 1222-1227.

Mori, Y., Higuchi, M., Hirabayashi, Y., Fukuda, M., and Gotoh, Y. (2008). JNK phosphorylates synaptotagmin-4 and enhances Ca2+-evoked release. Embo j 27, 76-87.

Moriguchi, T., Toyoshima, F., Masuyama, N., Hanafusa, H., Gotoh, Y., and Nishida, E. (1997). A novel SAPK/JNK kinase, MKK7, stimulated by TNFalpha and cellular stresses. Embo j 16, 7045-7053.

Mostowy, S., and Cossart, P. (2012). Septins: the fourth component of the cytoskeleton. Nat Rev Mol Cell Biol 13, 183-194.

Moughamian, A.J., and Holzbaur, E.L. (2012). Dynactin is required for transport initiation from the distal axon. Neuron 74, 331-343.

Moughamian, A.J., and Holzbaur, E.L. (2012). Synaptic vesicle distribution by conveyor belt. Cell 148, 849-851.

Moughamian, A.J., Osborn, G.E., Lazarus, J.E., Maday, S., and Holzbaur, E.L. (2013). Ordered recruitment of dynactin to the microtubule plus-end is required for efficient initiation of retrograde axonal transport. J Neurosci 33, 13190-13203.

Mukherjee, P.K., DeCoster, M.A., Campbell, F.Z., Davis, R.J., and Bazan, N.G. (1999). Glutamate receptor signaling interplay modulates stress-sensitive mitogen-activated protein kinases and neuronal cell death. J Biol Chem 274, 6493-6498. 
Mullis, K., Faloona, F., Scharf, S., Saiki, R., Horn, G., and Erlich, H. (1992). Specific enzymatic amplification of DNA in vitro: the polymerase chain reaction. 1986. Biotechnology (Reading, Mass) 24, 17-27.

Murthy, V.N., and De Camilli, P. (2003). Cell biology of the presynaptic terminal. Annu Rev Neurosci 26, 701-728.

Naito, S., and Ueda, T. (1985). Characterization of glutamate uptake into synaptic vesicles. J Neurochem 44, 99-109.

Nalefski, E.A., and Falke, J.J. (1996). The C2 domain calcium-binding motif: structural and functional diversity. Protein science : a publication of the Protein Society 5, 2375-2390.

Neco, P., Giner, D., del Mar Frances, M., Viniegra, S., and Gutierrez, L.M. (2003). Differential participation of actin- and tubulin-based vesicle transport systems during secretion in bovine chromaffin cells. The European journal of neuroscience 18, 733-742.

Ng, Y.-K., Lu, X., and Levitan, E.S. (2002). Physical mobilization of secretory vesicles facilitates neuropeptide release by nerve growth factor-differentiated PC12 Cells. The Journal of Physiology 542, 395-402.

Oheim, M., and Stuhmer, W. (2000). Tracking chromaffin granules on their way through the actin cortex. European biophysics journal : EBJ 29, 67-89.

Okada, Y., and Hirokawa, N. (2000). Mechanism of the single-headed processivity: diffusional anchoring between the K-loop of kinesin and the $\mathrm{C}$ terminus of tubulin. Proc Natl Acad Sci U S A 97, 640-645.

Okada, Y., Yamazaki, H., Sekine-Aizawa, Y., and Hirokawa, N. (1995). The neuron-specific kinesin superfamily protein KIF1A is a unique monomeric motor for anterograde axonal transport of synaptic vesicle precursors. Cell 81, 769-780.

Oliva, A.A., Jr., Atkins, C.M., Copenagle, L., and Banker, G.A. (2006). Activated c-Jun Nterminal kinase is required for axon formation. J Neurosci 26, 9462-9470.

Orci, L., Ravazzola, M., Amherdt, M., Perrelet, A., Powell, S.K., Quinn, D.L., and Moore, H.P.H. (1987). The trans-most cisternae of the Golgi complex: A compartment for sorting of secretory and plasma membrane proteins. Cell 51, 1039-1051.

Osborne, S.L., Herreros, J., Bastiaens, P.I., and Schiavo, G. (1999). Calcium-dependent oligomerization of synaptotagmins I and II. Synaptotagmins I and II are localized on the same synaptic vesicle and heterodimerize in the presence of calcium. J Biol Chem 274, 5966.

Overly, C.C., Rieff, H.I., and Hollenbeck, P.J. (1996). Organelle motility and metabolism in axons vs dendrites of cultured hippocampal neurons. J Cell Sci 109 ( Pt 5), 971-980.

Owald, D., and Sigrist, S.J. (2009). Assembling the presynaptic active zone. Curr Opin Neurobiol 19, 311-318. 
Pereda, A.E. (2014). Electrical synapses and their functional interactions with chemical synapses. Nat Rev Neurosci 15, 250-263.

Pollard, T.D., and Cooper, J.A. (2009). Actin, a central player in cell shape and movement. Science 326, 1208-1212.

Poo, M.-m. (2001). Neurotrophins as synaptic modulators. Nat Rev Neurosci 2, 24-32.

Rhee, J.S., Betz, A., Pyott, S., Reim, K., Varoqueaux, F., Augustin, I., Hesse, D., Sudhof, T.C., Takahashi, M., Rosenmund, C., et al. (2002). Beta phorbol ester- and diacylglycerol-induced augmentation of transmitter release is mediated by Munc13s and not by PKCs. Cell 108, 121-133.

Rizzoli, S.O. (2014). Synaptic vesicle recycling: steps and principles. EMBO J 33, 788-822.

Roberts, A.J., Kon, T., Knight, P.J., Sutoh, K., and Burgess, S.A. (2013). Functions and mechanics of dynein motor proteins. Nat Rev Mol Cell Biol 14, 713-726.

Rudolf, R. (2003). Myosin Va facilitates the distribution of secretory granules in the F-actin rich cortex of PC12 cells. Journal of Cell Science 116, 1339-1348.

Rudolf, R., Salm, T., Rustom, A., and Gerdes, H.H. (2001). Dynamics of immature secretory granules: role of cytoskeletal elements during transport, cortical restriction, and F-actindependent tethering. Mol Biol Cell 12, 1353-1365.

Sabo, S.L., Gomes, R.A., and McAllister, A.K. (2006). Formation of presynaptic terminals at predefined sites along axons. J Neurosci 26, 10813-10825.

Sadakata, T., and Furuichi, T. (2009). Developmentally regulated Ca2+-dependent activator protein for secretion 2 (CAPS2) is involved in BDNF secretion and is associated with autism susceptibility. Cerebellum 8, 312-322.

Saiki, R.K., Gelfand, D.H., Stoffel, S., Scharf, S.J., Higuchi, R., Horn, G.T., Mullis, K.B., and Erlich, H.A. (1988). Primer-directed enzymatic amplification of DNA with a thermostable DNA polymerase. Science $239,487-491$.

Sankaranarayanan, S., and Ryan, T.A. (2000). Real-time measurements of vesicle-SNARE recycling in synapses of the central nervous system. Nat Cell Biol 2, 197-204.

Scalettar, B.A. (2006). How neurosecretory vesicles release their cargo. Neuroscientist 12, 164-176.

Schägger, H., and von Jagow, G. (1987). Tricine-sodium dodecyl sulfate-polyacrylamide gel electrophoresis for the separation of proteins in the range from 1 to $100 \mathrm{kDa}$. Analytical Biochemistry 166, 368-379.

Schroeder, H.W., 3rd, Mitchell, C., Shuman, H., Holzbaur, E.L., and Goldman, Y.E. (2010). Motor number controls cargo switching at actin-microtubule intersections in vitro. Curr Biol 20, 687-696. 
Shakiryanova, D., Tully, A., and Levitan, E.S. (2006). Activity-dependent synaptic capture of transiting peptidergic vesicles. Nat Neurosci 9, 896-900.

Shin, H., Wyszynski, M., Huh, K.H., Valtschanoff, J.G., Lee, J.R., Ko, J., Streuli, M., Weinberg, R.J., Sheng, M., and Kim, E. (2003). Association of the kinesin motor KIF1A with the multimodular protein liprin-alpha. J Biol Chem 278, 11393-11401.

Smith, A.J., Pfeiffer, J.R., Zhang, J., Martinez, A.M., Griffiths, G.M., and Wilson, B.S. (2003). Microtubule-dependent transport of secretory vesicles in RBL-2H3 cells. Traffic 4, 302-312.

Soppina, V., Norris, S.R., Dizaji, A.S., Kortus, M., Veatch, S., Peckham, M., and Verhey, K.J. (2014). Dimerization of mammalian kinesin-3 motors results in superprocessive motion. Proc Natl Acad Sci U S A 111, 5562-5567.

Sorra, K.E., Mishra, A., Kirov, S.A., and Harris, K.M. (2006). Dense core vesicles resemble active-zone transport vesicles and are diminished following synaptogenesis in mature hippocampal slices. Neuroscience 141, 2097-2106.

Stagi, M., Gorlovoy, P., Larionov, S., Takahashi, K., and Neumann, H. (2006). Unloading kinesin transported cargoes from the tubulin track via the inflammatory c-Jun $\mathrm{N}$-terminal kinase pathway. FASEB J 20, 2573-2575.

Stepanova, T., Smal, I., van Haren, J., Akinci, U., Liu, Z., Miedema, M., Limpens, R., van Ham, M., van der Reijden, M., Poot, R., et al. (2010). History-dependent catastrophes regulate axonal microtubule behavior. Curr Biol 20, 1023-1028.

Sudhof, T.C. (2002). Synaptotagmins: why so many? J Biol Chem 277, 7629-7632.

Sudhof, T.C. (2004). The synaptic vesicle cycle. Annu Rev Neurosci 27, 509-547.

Sudhof, T.C. (2012). The presynaptic active zone. Neuron 75, 11-25.

Taylor, A.M., Wu, J., Tai, H.C., and Schuman, E.M. (2013). Axonal translation of beta-catenin regulates synaptic vesicle dynamics. J Neurosci 33, 5584-5589.

Thomas, C., Rousset, R., and Noselli, S. (2009). JNK signalling influences intracellular trafficking during Drosophila morphogenesis through regulation of the novel target gene Rab30. Dev Biol 331, 250-260.

Thomas, D.M., Ferguson, G.D., Herschman, H.R., and Elferink, L.A. (1999). Functional and biochemical analysis of the C2 domains of synaptotagmin IV. Mol Biol Cell 10, 2285-2295.

Ting, J.T., Kelley, B.G., and Sullivan, J.M. (2006). Synaptotagmin IV does not alter excitatory fast synaptic transmission or fusion pore kinetics in mammalian CNS neurons. J Neurosci $26,372-380$.

Trinczek, B., Ebneth, A., Mandelkow, E.M., and Mandelkow, E. (1999). Tau regulates the attachment/detachment but not the speed of motors in microtubule-dependent transport of single vesicles and organelles. J Cell Sci 112 ( Pt 14), 2355-2367. 
Trueta, C., and De-Miguel, F.F. (2012). Extrasynaptic exocytosis and its mechanisms: a source of molecules mediating volume transmission in the nervous system. Front Physiol 3, 319.

Tsuboi, T., and Rutter, G.A. (2003). Multiple forms of "kiss-and-run" exocytosis revealed by evanescent wave microscopy. Curr Biol 13, 563-567.

Ullrich, B., Li, C., Zhang, J.Z., McMahon, H., Anderson, R.G., Geppert, M., and Sudhof, T.C. (1994). Functional properties of multiple synaptotagmins in brain. Neuron 13, 1281-1291.

Vallee, R.B., Seale, G.E., and Tsai, J.W. (2009). Emerging roles for myosin II and cytoplasmic dynein in migrating neurons and growth cones. Trends Cell Biol 19, 347-355.

van de Bospoort, R., Farina, M., Schmitz, S.K., de Jong, A., de Wit, H., Verhage, M., and Toonen, R.F. (2012). Munc13 controls the location and efficiency of dense-core vesicle release in neurons. J Cell Biol 199, 883-891.

Varoqueaux, F., Sigler, A., Rhee, J.S., Brose, N., Enk, C., Reim, K., and Rosenmund, C. (2002). Total arrest of spontaneous and evoked synaptic transmission but normal synaptogenesis in the absence of Munc13-mediated vesicle priming. Proc Natl Acad Sci U S A 99, 90379042.

Verhage, M., and Sorensen, J.B. (2008). Vesicle docking in regulated exocytosis. Traffic 9, $1414-1424$

Vician, L., Lim, I.K., Ferguson, G., Tocco, G., Baudry, M., and Herschman, H.R. (1995). Synaptotagmin IV is an immediate early gene induced by depolarization in PC12 cells and in brain. Proc Natl Acad Sci U S A 92, 2164-2168.

von Poser, C., Ichtchenko, K., Shao, X., Rizo, J., and Sudhof, T.C. (1997). The evolutionary pressure to inactivate. A subclass of synaptotagmins with an amino acid substitution that abolishes Ca2+ binding. J Biol Chem 272, 14314-14319.

Wakatsuki, T., Schwab, B., Thompson, N.C., and Elson, E.L. (2001). Effects of cytochalasin D and latrunculin B on mechanical properties of cells. J Cell Sci 114, 1025-1036.

Wang, C.T., Grishanin, R., Earles, C.A., Chang, P.Y., Martin, T.F., Chapman, E.R., and Jackson, M.B. (2001). Synaptotagmin modulation of fusion pore kinetics in regulated exocytosis of dense-core vesicles. Science 294, 1111-1115.

Wang, C.T., Lu, J.C., Bai, J., Chang, P.Y., Martin, T.F., Chapman, E.R., and Jackson, M.B. (2003). Different domains of synaptotagmin control the choice between kiss-and-run and full fusion. Nature 424, 943-947.

Watanabe, S., Rost, B.R., Camacho-Perez, M., Davis, M.W., Sohl-Kielczynski, B., Rosenmund, C., and Jorgensen, E.M. (2013). Ultrafast endocytosis at mouse hippocampal synapses. Nature 504, 242-247.

Westerlund, N., Zdrojewska, J., Padzik, A., Komulainen, E., Bjorkblom, B., Rannikko, E., Tararuk, T., Garcia-Frigola, C., Sandholm, J., Nguyen, L., et al. (2011). Phosphorylation of 
SCG10/stathmin-2 determines multipolar stage exit and neuronal migration rate. Nat Neurosci 14, 305-313.

Whalley, K. (2008). Signal transduction: New tricks for old JNKs. Nature Reviews Neuroscience 9, 164-165.

Wisniewski, J.R., Nagaraj, N., Zougman, A., Gnad, F., and Mann, M. (2010). Brain phosphoproteome obtained by a FASP-based method reveals plasma membrane protein topology. Journal of proteome research 9, 3280-3289.

Wong, M.Y., Cavolo, S.L., and Levitan, E.S. (2015). Synaptic neuropeptide release by dynamin-dependent partial release from circulating vesicles. Mol Biol Cell 26, 2466-2474.

Wong, M.Y., Zhou, C., Shakiryanova, D., Lloyd, T.E., Deitcher, D.L., and Levitan, E.S. (2012). Neuropeptide delivery to synapses by long-range vesicle circulation and sporadic capture. Cell 148, 1029-1038.

Wong, R.W., Setou, M., Teng, J., Takei, Y., and Hirokawa, N. (2002). Overexpression of motor protein KIF17 enhances spatial and working memory in transgenic mice. Proc Natl Acad Sci U S A 99, 14500-14505.

Wong, Y.H., Lee, C.M., Xie, W., Cui, B., and Poo, M.M. (2015). Activity-dependent BDNF release via endocytic pathways is regulated by synaptotagmin- 6 and complexin. Proc Natl Acad Sci U S A 112, E4475-4484.

Wu, L.G., Hamid, E., Shin, W., and Chiang, H.C. (2014). Exocytosis and endocytosis: modes, functions, and coupling mechanisms. Annual review of physiology 76, 301-331.

Wu, Y.E., Huo, L., Maeder, C.I., Feng, W., and Shen, K. (2013). The balance between capture and dissociation of presynaptic proteins controls the spatial distribution of synapses. Neuron 78, 994-1011.

Xia, X., Lessmann, V., and Martin, T.F. (2009). Imaging of evoked dense-core-vesicle exocytosis in hippocampal neurons reveals long latencies and kiss-and-run fusion events. J Cell Sci 122, 75-82.

$\mathrm{Xu}, \mathrm{T}$. , and $\mathrm{Xu}, \mathrm{P}$. (2008). Searching for molecular players differentially involved in neurotransmitter and neuropeptide release. Neurochem Res 33, 1915-1919.

Yonekawa, Y., Harada, A., Okada, Y., Funakoshi, T., Kanai, Y., Takei, Y., Terada, S., Noda, T., and Hirokawa, N. (1998). Defect in synaptic vesicle precursor transport and neuronal cell death in KIF1A motor protein-deficient mice. J Cell Biol 141, 431-441.

Yoshihara, M., and Montana, E.S. (2004). The synaptotagmins: calcium sensors for vesicular trafficking. Neuroscientist 10, 566-574.

Zahn, T.R., Angleson, J.K., MacMorris, M.A., Domke, E., Hutton, J.F., Schwartz, C., and Hutton, J.C. (2004). Dense core vesicle dynamics in Caenorhabditis elegans neurons and the role of kinesin UNC-104. Traffic 5, 544-559. 
Zhang, Q., Fukuda, M., Van Bockstaele, E., Pascual, O., and Haydon, P.G. (2004). Synaptotagmin IV regulates glial glutamate release. Proc Natl Acad Sci U S A 101, 94419446.

Zhang, Z., Wu, Y., Wang, Z., Dunning, F.M., Rehfuss, J., Ramanan, D., Chapman, E.R., and Jackson, M.B. (2011). Release mode of large and small dense-core vesicles specified by different synaptotagmin isoforms in PC12 cells. Mol Biol Cell 22, 2324-2336.

Zhong, G., He, J., Zhou, R., Lorenzo, D., Babcock, H.P., Bennett, V., and Zhuang, X. (2014). Developmental mechanism of the periodic membrane skeleton in axons. Elife 3.

Zhou, F.F., Xue, Y., Chen, G.L., and Yao, X. (2004). GPS: a novel group-based phosphorylation predicting and scoring method. Biochem Biophys Res Commun 325, 1443-1448. 


\section{Abbreviations}

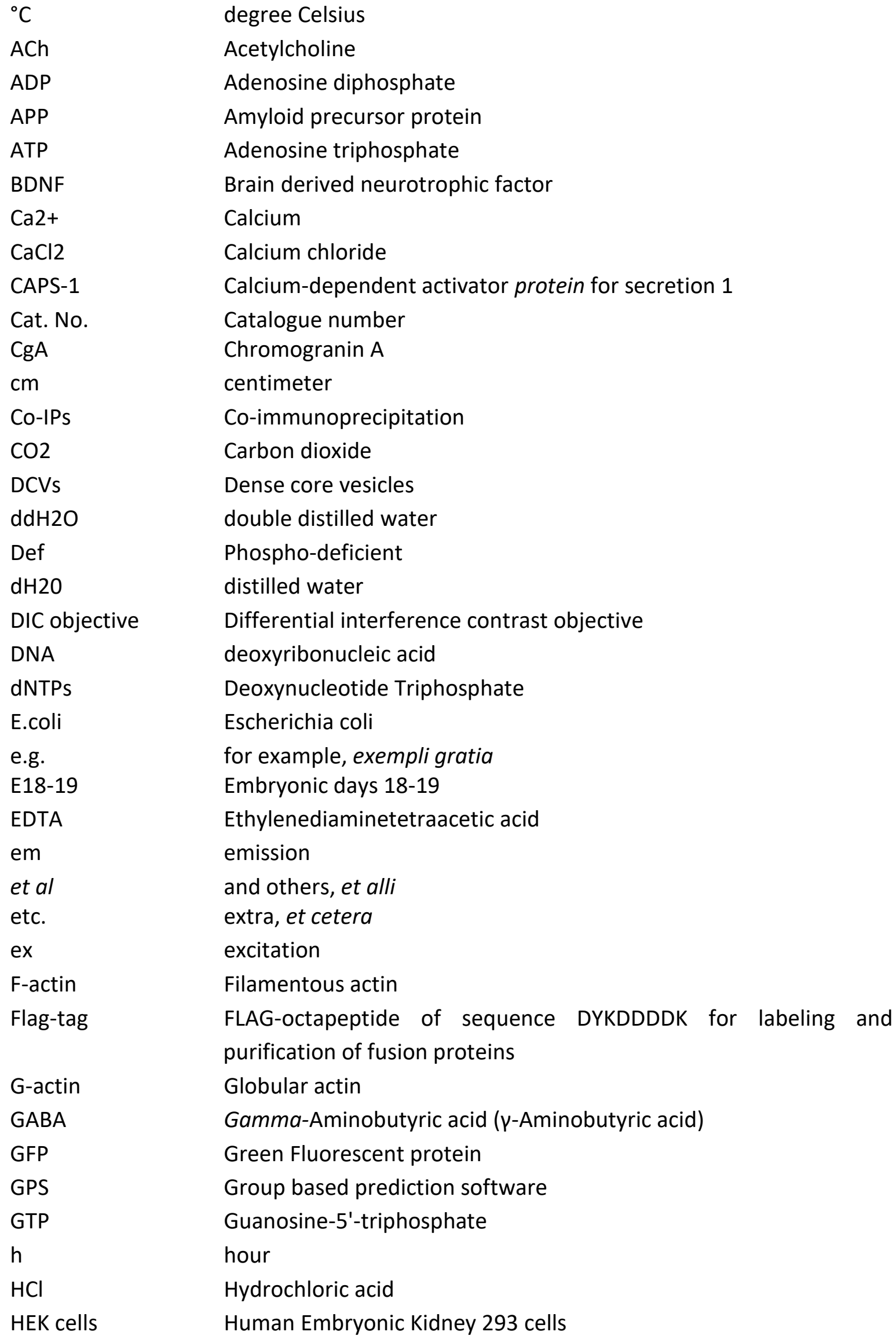




\begin{tabular}{|c|c|}
\hline HRP & Horse radish peroxidase \\
\hline i.e. & that is, id est \\
\hline JIP1 & JNK interacting protein 1 \\
\hline JNK & c-Jun N-terminal kinase \\
\hline $\mathrm{KCl}$ & Potassium Chloride \\
\hline $\mathrm{kDa}$ & kilodalton \\
\hline KO & Knockout \\
\hline LB & Lysogeny broth \\
\hline $\mathrm{m}$ & meter \\
\hline Map2 & Microtubule associated protein 2 \\
\hline MAPK & Mitogen activated protein kinase \\
\hline MAPKK & Mitogen activated protein kinase kinase \\
\hline MAPKKK & Mitogen activated protein kinase kinase kinase \\
\hline mg & milligram \\
\hline $\mathrm{MgCl} 2$ & magnesium chloride \\
\hline $\min$ & minutes \\
\hline $\mathrm{ml}$ & millilitres \\
\hline $\mathrm{mM}$ & millimolar \\
\hline MTs & Microtubules \\
\hline $\mathrm{Na}+$ & Sodium ion \\
\hline $\mathrm{Na} 2 \mathrm{HPO} 4$ & Sodium hydrogen phosphate \\
\hline $\mathrm{NaCl}$ & Sodium chloride \\
\hline NB+ & Neurobasal plus media (with B-27 and Glutamax supplements) \\
\hline NGF & Nerve Growth Factor \\
\hline $\mathrm{Nh} 4 \mathrm{Cl}$ & Ammonium chloride \\
\hline $\mathrm{nm}$ & nanometer \\
\hline NMJ & Neuromuscular junction \\
\hline NPY & Neuropeptide Y \\
\hline NSF & $N$-ethylmaleimide-sensitive factor \\
\hline NTs & Neurotransmitters \\
\hline PO & Postnatal day 0 \\
\hline PAGE & Polyacrylamide Gel Electrophoresis \\
\hline PBS & Phosphate buffer saline \\
\hline PC-12 cells & Pheochromocytoma cells \\
\hline PCR & Polymerase chain reaction \\
\hline PDL & Poly-D-lysine \\
\hline PEI & Polyethylenimine \\
\hline PSD-95 & Postsynaptic density -95 \\
\hline Rab-GDI & Rab GDP dissociation inhibitor \\
\hline rER & rough endoplasmic reticulum \\
\hline RFP & Red fluorescent protein \\
\hline Rpm & revolution per minute \\
\hline
\end{tabular}


$\mathrm{S}$

S135

SAPK1

SDS

SNAP-25

SNARE

SVS

Syb 2

Syt4 -/-

Syt4

Syts

TAE

TPY motif

UV

$\mathrm{V}$

VAMP2

vGAT

vGluT

$w / v$

WT

$\mu l$

$\mu \mathrm{M}$ second

Serine at 135 position

Stress activated protein kinase 1

Sodium dodecyl sulphate

Synaptosomal-Associated Protein 25

Soluble NSF Attachment Protein Receptor

Synaptic vesicles

Synaptobrevin 2

Synaptotagmin 4 knockout

Synaptotagmin 4

Synaptotagmins

Tris base, acetic acid and EDTA buffer

Threonine-Proline-Tyrosine motif

ultraviolet

voltage

Vesicle associated membrane protein 2

vesicular GABA transporter

vesicular Glutamate transporter

weight/volume

Wild type

microliter

micromolar 



\section{Acknowledgments}

Oh! This is the part, which I think will come automatically "without any literature search". I have so many people to thank who helped in walking me through this journey happily. It was truly a fun rollercoaster ride because of you all being there.

First of all, thank you Camin Dean, my PhD supervisor, who actually is not just my scientific guide but also, is someone from whom I have learned a lot in life. You have immensely influenced my love and experience for research. You are an amazing person; always keen to help everyone in any way possible. Your way of doing science, approach towards dealing with situations, ideas for development and progress of your students have always motivated me to perform my best. I totally loved working with you since my lab rotation till this time. I can't thank you enough Camin for being there for me whenever I needed you. Your office doors were always opened for me to discuss scientific or non-scientific topics... which I can never forget. I couldn't have asked for anyone better than you for guiding me in my PhD.

I am also really thankful to Prof. Dr. Nils Brose and Prof. Dr. Reinhard Jahn for being in my core committee. Your timely inputs, suggestions and ideas were very important for driving this project to this level. I totally loved and gained a lot from our discussions during meetings. It was amazing to have eminent scientists like you both in my PhD thesis committee for feedbacks and comments.

Next, I would like to acknowledge my amazing lab mates. Trust me, all this effort and work was only possible because of such a fun and cordial environment in the lab. Thank you for bearing with my singing and laughing....You guys were just fun to hang around with...Joaquin (my first lab mate, officemate cum really good "Sausailitos" lover friend), Markus (batch mate cum friend for more than 5 years now with whom I started my PhD!), Anne ("Blue" "organized" "coffee" girl with smile and fun ideas), Ankit ( "Junior PhD"..hehe! who's love for science and work was always inspiring. With you it was the fun "desi" connection in the lab), Charlie ("scientist" who loves cooking and eating Indian food), Saheeb (Biochemistry expert who always took time for "Syt4"), Binu ("LTP expert"... with whom discussing about India is always fun), Katja (known you from just few months, but your love and care for me is heartfelt). Thank you guys for being there! Without you guys around, working would not have been that much fun. 
I would also like to thank Dr. Yo Shinoda for being my microscopy guru. You taught me fine details about this technique which I love doing. I would also like to thank my collaborators.... Dr. Jonathan Ting, for giving us phopshomutants constructs which was the key for whole project. Matlab expert, Michael Seibrecht for writing customized codes whenever I came up with something random to try! Mahi, from Dr. Jahn's lab for her kind support and time in helping me with in vitro kinase assay. Vicky, my lab rotation student for giving me some nice trafficking results. Thank you all for being there and being associated with this project. I would also like to extend my acknowledgement to the entire office holders (especially Christiane), GGNB, IT technicians etc. who helped me whenever needed. I am also really thankful to Prof. Dr. Thomas Dresbach and his lab and all ENI members including animal house caretakers for their time and support whenever required.

My journey for PhD started because of MSc/PhD IMPRS Neuroscience programme. To be a part of this programme was a privilege. Coming away from home just after bachelors raised so many doubts and questions...but thanks to Prof. Michael Horner, Sandra Drube and Mirja for taking care of each and every minute thing during this stay. The feeling of being from the programme, having amazing fellow mates from so many different cultures and countries is just amazing. Thank you; especially 2010-11 batch for giving such a great start and company in this journey. Be it the coffee group "Ants, Annie, Bekir and David" or my dear friends Zohreh and IIma, thank you for being there for me whenever needed.

Getting "homesick" was not an option here just because of finding a home away from home. "Crazy Indians in Goettingen" was my family here who stood beside me in every situation. Great days, happy days, so so days or sad days.. they know it all of my PhD life. All the quality time spent together, cooking food, movie nights, game nights, trips in Europe helped a lot to balance work and life...: "Kundan, Veena and Heena" are the three names always on my call list. I don't remember a day without talking to them. Kunds...Knowing you was by chance, but now sharing such a unique friendship with you is simply amazing. You are the best friend one can have. Your jokes, calling me by "101 names", motivating me in "a unique way" to conquer anything in anytime, being there with me through thick and thin; helping me in compiling my thesis made it possible to live here with a big smile. Veens.. "schwester" you are actually one of the reason for me being here. I still remember your mail describing Goettingen to me. You are so loving and caring that I always felt secured and loved next to you. Be it any situation, you understand every bit of me without the need of describing you. And of course thank you for giving me JNK inhibitor SP600125..:) Heens..."honey" discussing with you any situations 
(scientific or non-scientific..:P) always gave me strength to deal with them during this time. I totally love your attitude and approach towards life. Your care and support is something, which kept me going happily in this journey.

The list of friends is long and never ending. I would like to sum them up shortly. Soham "my khurafaati brother" thank you for understanding all my "Ghuchu-muchu" situations. Pawan, thank you for being that 1 am friend (literary) and for being there by my side "always". Sumir, thank you for taking care as a big bro. Avani, the all-rounder thanks dost for being the ENI-floor mate too. It was a huge support for me to have you there. Panchi, my genius "sundar" astrophysicist... Thanks for coming to my life. Upasana, Priyanka, Arnab without you guys this would not have been such a smooth journey. Mayur thanks a lot for helping me out; be it choosing lab for masters or now, for reading parts of my thesis. Danke Somu, for taking care of my beautiful Europe travels...). Apart from the names mentioned, thanks to all Indian connections seniors, juniors, Nirmiti mates, Wurzburg gang, everyone who brought smile to my face during this time. I might miss some names, but trust me am thankful to everyone for being there for me.

My teachers and mentors thanks for making me a person what I am today. Last but not the least; I wouldn't have come this far if it was not my lovely family - papa, mummy, chachu and bhai; backing me up. The words fall short to describe what you guys mean to me. Your unconditional love and faith in me is the most motivating and inspiring thing in my life. Papa you consider me as "bade ustaad" bestow extra responsibility on me to fulfill every task $100 \%$ and that too happily. Your way of living is inspiring. I am so proud of you! Maa, your love and care are beyond words. You are my heart and soul, the reason for what I am today. Chachu, you are my pillar of strength and a person whom I look up to. Bhai, my veera, you are the best brother cum buddy I could have. Your immense confidence, belief and love for me have always motivated me to go ahead in my life. My grandma "Amma", you are being missed but your love and blessings for me is encouraging me in every walk of life. I love you guys more than anything. Thank you Almighty! for being there as a force to drive me through this beautiful journey so far. The fascination of understanding "how our brain functions?" is what actually made it possible! 



\section{Curriculum Vitae}

\section{Personal Data}

\author{
Last Name \\ First Name \\ Date of Birth \\ Place of Birth \\ Nationality \\ Address \\ Telephone \\ E-Mail
}

\section{Education}

4/2012 - present

$9 / 2010-3 / 2012$

$3 / 2007-3 / 2010$

\section{Research experience}

4/2012 - present

$9 / 2011-3 / 2012$

$5 / 2011-6 / 2011$

$3 / 2011-4 / 2011$
Bharat

Vinita

08 ${ }^{\text {th }}$ December 1989

Delhi, India

Indian

Philipp-Reis-Strasse 3/25, Göttingen, 37075 Germany +49-(0) 17670294514

vinitabharat89@gmail.com,v.bharat@eni-g.de

PhD in Neuroscience, Department of Trans-Synaptic Signaling, European Neuroscience Institute (ENI), Göttingen, Germany Supervisor: Camin Dean, PhD, M.Sc/PhD Neuroscience Program International Max Planck Research School (IMPRS), University of Göttingen, Germany B.Sc.(Honors) Biochemistry,Sri Venkateswara College University of Delhi, Delhi, India

PhD thesis - "Phosphorylation of Synaptotagmin 4 captures transiting dense core vesicles at active synapses" Camin Dean, PhD, ENI, Göttingen,Germany Master's Thesis - "Characterization of reporters for dense core versus synaptic vesicle exocytosis in neurons." Camin Dean,PhD, ENI, Göttingen, Germany Research Intern - "Effect of Brain- derived neurotrophic factor (BDNF) on presynaptic function"

Dr. Yo Shinoda and Camin Dean, PhD, ENI, Göttingen, Germany

Research Intern - "Identification and analysis of Mechanosensory specific enhancer of Fam38b" 
$1 / 2011-2 / 2011$

$6 / 2009$

$5 / 2009$

$8 / 2009$

$5 / 2009-6 / 2009$

$2007-2008$

\section{Scholarships/Awards}

2014

2013

$2010-2011$

2008-2009

\section{Language skills}

\section{English}

Hindi

German
Dr. Till Marquardt, Department of Development Neurobiology, ENI, Göttingen, Germany

Research Intern - "Expression of Eag1 channels in cerebellar Purkinje cell"

Dr. Luis Pardo, MPI- experimental medicine, Göttingen, Germany

Summer internship in Central Research Institute (CRI), India

Summer Internship in Surya pharmaceuticals, India Add on Course in Bioinformatics, University of Delhi, India Summer Internship Project- "Duchenne Muscular Dystrophy"

Dr. Jayesh Sheth, Institute of Human Genetics, India Add on course in Nanotechnology, University of Delhi, India

Travel grant for the "Gordon Research Conference for cell biology of the neuron" in Waterville Valley, $\mathrm{NH}$, USA Travel grant for the "Society for Neuroscience 2013" conference in San Diego, USA from Goettingen Graduate School for Neurosciences, Biophysics and Molecular Biosciences (GGNB)

Stipend of the Excellence Foundation for the promotion of Max Planck Society

Certificate of merit, second rank in the Delhi University

\section{Teaching Experience}

Have experience of supervising six students for lab rotation projects during my PhD. 


\section{Conferences contributions}

$>$ Poster "Phosphorylation of Syt4 regulates dense core vesicle trafficking in hippocampal neurons" in ENI-MIT symposium 2015

$>$ Poster "Effect of phosphorylation of Syt4 on DCV trafficking and fusion in hippocampal neurons" in Gordon Research Conference of cell biology of the neuron 2014 (GRC 2014).

$>$ Poster "Simultaneous Visualization of dense core versus synaptic vesicles in hippocampal neurons" presented in Society of Neuroscience 2013 (SFN 2013) and GGNB Science Day (2013).

$>$ Talk on "Is our brain plastic? If so, how?" in Third Young Investigator's Meeting 2014

$>$ Talk on "Effect of phosphorylation of syt4 on DCV trafficking and fusion in hippocampal neurons" in International Max Planck Research School (IMPRS) Neuroscience retreat in 2013 and 2014.

\section{Publications}

Shinoda, Y., Ahmed, S., Ramachandran, B., Bharat, V., Brockelt, D., Altas, B., \& Dean, C. (2014). BDNF enhances spontaneous and activity-dependent neurotransmitter release at excitatory terminals but not at inhibitory terminals in hippocampal neurons. Frontiers in Synaptic Neuroscience, 6, 27.

Dean, C., Dunning, F. M., Liu, H., Bomba-Warczak, E., Martens, H., Bharat V, Ahmed S,Chapman, E. R. (2012). Axonal and dendritic synaptotagmin isoforms revealed by a pHluorin-syt functional screen. Molecular Biology of the Cell, 23(9), 1715-1727. 

